\title{
Shadow trees of Mandelbrot sets
}

\author{
by \\ Virpi Kauko (Jyväskylä)
}

\begin{abstract}
The topology and combinatorial structure of the Mandelbrot set $\mathcal{M}^{d}$ (of degree $d \geq 2$ ) can be studied using symbolic dynamics. Each parameter is mapped to a kneading sequence, or equivalently, an internal address; but not every such sequence is realized by a parameter in $\mathcal{M}^{d}$. Thus the abstract Mandelbrot set is a subspace of a larger, partially ordered symbol space, $\Lambda^{d}$. In this paper we find an algorithm to construct "visible trees" from symbolic sequences which works whether or not the sequence is realized. We use this procedure to find a large class of addresses that are nonrealizable, and to prove that all such trees in $\Lambda^{d}$ actually satisfy the Translation Principle (in contrast to $\mathcal{M}^{d}$ ). We also study how the existence of a hyperbolic component with a given address depends on the degree $d$ : addresses can be sorted into families so that at least one address of each family is realized for sufficiently large $d$.
\end{abstract}

\section{INTRODUCTION}

The generalized Mandelbrot sets $\mathcal{M}^{d}$ are interesting not only from the complex dynamical and fractal geometric points of view, but also for their combinatorial properties. After some observations on the general shape and size of the sets in Section 1, we focus on the combinatorics.

We use the symbolic dynamical theory developed in [D-H], [Do-1], [Thu], [Mil-2], [Ate], [B-K], [Pen], [L-S-1] (etc.) and summarize it in Sections 2-4; see also [Kel], [Do-2]. Given an angle, one finds its kneading sequence and translates it to an internal address using the Lau-Schleicher Algorithm (LSA). This tells us where in $\partial \mathcal{M}^{d}$ the external ray with that angle lands at (or accumulates to). Every parameter in $\mathcal{M}^{d}$ is thus attached to an address $n_{1}\left(s_{1}\right) \longmapsto n_{2}\left(s_{2}\right) \longmapsto \ldots$, where the rough address $n_{1} \longmapsto n_{2} \longmapsto \ldots$ is a strictly increasing sequence of integers starting with $n_{1}=1$, and the sector numbers $s_{i}$ are in $\mathbb{Z}_{d} \backslash\{0\}$ for all $i \in \mathbb{N}$.

An interesting fact is that not every such sequence occurs as internal addresses of parameters in $\mathcal{M}^{d}$. This raises the fundamental question: which

2000 Mathematics Subject Classification: Primary 37F20; Secondary 37B10, 05C05.

The author was supported by Finnish Academy projects \#39788 and \#23795 
formal addresses are realizable and which are not? We give some partial answers to that. A characterization in the quadratic case $(d=2)$ has been done by Bruin and Schleicher [B-S]; they use Hubbard trees to study the dynamics determined by the address, whereas our arguments are mainly based on the Structure Theorems, i.e., knowledge about landing of rational external parameter rays [D-H], [Mil-2], [Sch-2], [Ebe], [Rie].

If we denote by $\simeq$ the equivalence relation that identifies parameters sharing an internal address, the quotient space $\mathcal{M}^{d} / \simeq$ is actually a proper subset of a larger symbol space $\Lambda^{d}$ of formal addresses, which we study in Section 5. Using the fact that LSA is well defined regardless of the existence of a component with a given internal address, we extend the combinatorial tree structure of the Mandelbrot set into the space $\Lambda^{d}$.

The internal address (or equivalently, kneading sequence) not only tells us which way to walk from the origin in order to reach a parameter with a given external angle [L-S-1]; we find that it also tells one which components are ahead if one continues away from the origin. In $\S 6$ we develop a new algorithm (based on LSA) to construct the tree of formal addresses that are visible from a given base. The hyperbolic components realizing them in $\mathcal{M}^{d}$-if such exist! - are then visible from the base sector in the sense of [L-S-1]. Moreover, our tree-growing algorithm can be used to construct shadow trees which only contain nonrealizable formal addresses, or "nonexistent" components. Finding shadow trees within formal trees thus helps to distinguish existent components from nonexistent ones.

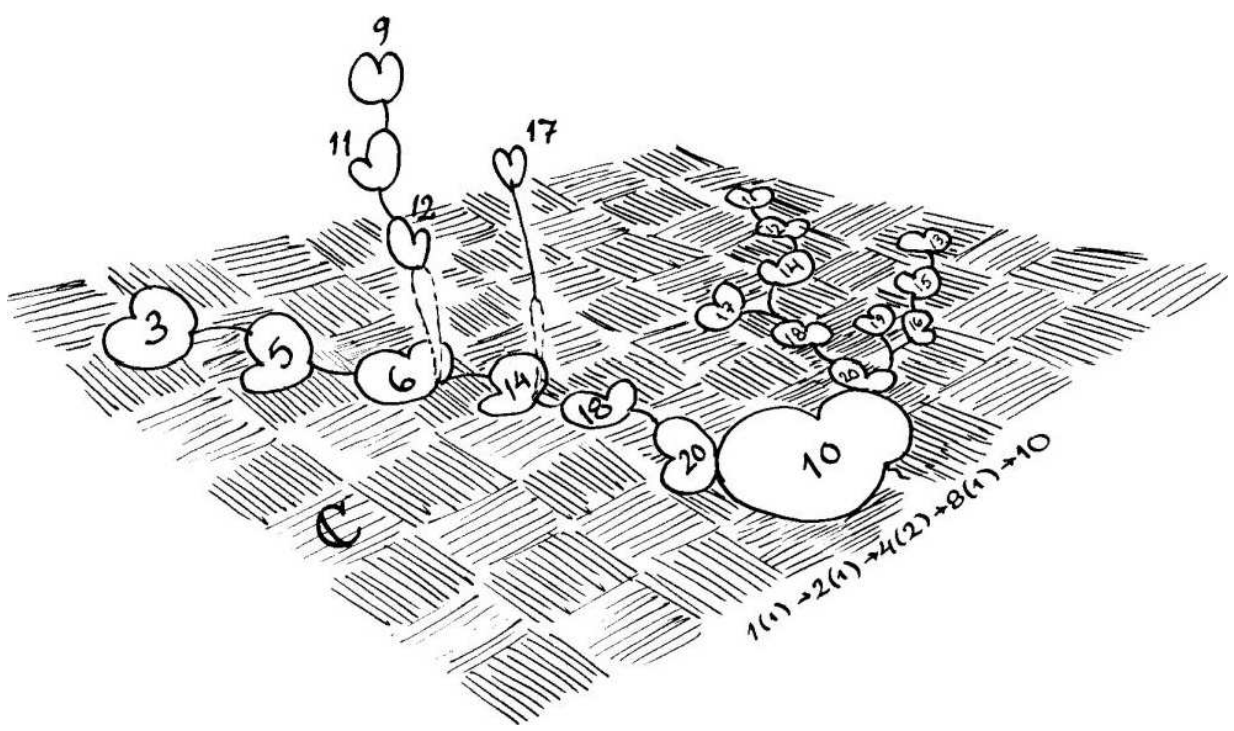

Fig. A 
Another main result of this paper is that the "Translation Principle" (3.6) of combinatorial equivalence holds for formal trees (6.14) even though it is not true in general for real visible trees. In this sense the symbol space $\Lambda^{d}$ is more regular than the Mandelbrot set.

In $\S 7$ we use results from $\S 6$ to prove a "folklore theorem" about wakewidths of hyperbolic satellite components. This result is needed in $\S 8$, as we study how the realizability of formal addresses depends on the degree $d$. We find that every rough address is realized with some set of sector numbers, for a sufficiently large $d$ depending on the length of the address.

The nonexistent parameters thus form a "shadow space" with similar tree structures to the real one. Figure A shows an example of a ten-periodic hyperbolic component in $\mathcal{M}_{3}$ with formal trees stemming from its two $1 / 2$ satellites. In contrast to the natural world, here the actual visible trees lie flat on the complex plane while the shadow trees are sticking up to another dimension.

Acknowledgements. I am grateful to many people for their support. Professor Kari Astala introduced me to complex dynamics, and he and Professors Pekka Koskela and Pertti Mattila gave me the opportunity to work in their Finnish Academy projects. Peter Haïssinsky and Kirsi Peltonen have carefully read the manuscript of this paper; their comments helped me improve it. Discussions with Dierk Schleicher, Bodil Branner, and others have also given me valuable ideas and advice. Special thanks are due to Tapani Tarvainen, who wrote computer programs to actually carry out the visible tree algorithm.

\section{GEOMETRIC PROPERTIES OF $\mathcal{M}^{d}$}

The Mandelbrot set $\mathcal{M}^{d}$ of degree $d$ is defined as the set of parameters $c \in \mathbb{C}$ for which the Julia set of the polynomial $P_{c}: z \mapsto z^{d}+c$ is connected; equivalently,

$$
\mathcal{M}^{d}:=\left\{c \in \mathbb{C}: P_{c}^{n}(0):=P_{c} \circ \ldots \circ P_{c}(0) \nrightarrow \infty \text { as } n \rightarrow \infty\right\} .
$$

(By a classical result of Fatou and Julia, the Julia set of a polynomial is connected if and only if all its finite critical points have bounded orbits; zero is the only finite critical point of $P_{c}[\mathrm{C}-\mathrm{G}, \mathrm{III}]$, [Mil-1].)

1.2. Hyperbolic components and sectors. The multiplier of a $k$ periodic orbit $\left\{z_{1}, \ldots, z_{k}\right\}$ (where $z_{j+1}=z_{j}^{d}+c$ and $j \in \mathbb{Z}_{k}$ ) of $P_{c}$ is

$$
\lambda(c):=\left(P_{c}^{k}\right)^{\prime}\left(z_{1}\right)=P_{c}^{\prime}\left(z_{k}\right) \cdot \ldots \cdot P_{c}^{\prime}\left(z_{1}\right)=d^{k} \cdot\left(z_{1} \cdot \ldots \cdot z_{k}\right)^{d-1} .
$$

The orbit is attracting if $|\lambda|<1$, neutral if $|\lambda|=1$ (in particular, parabolic if $\lambda=e^{i 2 \pi \phi}$ with $\phi$ in $\mathbb{Q}$ ), and repelling if $|\lambda|>1$. For every $k \in \mathbb{N}$, the set

$$
\mathcal{H}^{d}(k):=\left\{c \in \mathbb{C}: P_{c} \text { has an attracting } k \text {-periodic orbit }\right\}
$$


is well known to be an open subset of $\mathcal{M}^{d}$, and its connected components are called the hyperbolic components. Each polynomial $P_{c}$ with $c$ in some hyperbolic component $\mathcal{H} \subset \mathcal{H}^{d}(k)$ has a unique $k$-periodic orbit whose multiplier has absolute value less than one; that is, $\lambda(c)$ belongs to the unit disk $\mathbb{D}$. The number $k$ is also called the period of the component $\mathcal{H}$, denoted by $\langle\mathcal{H}\rangle$.

The Douady-Hubbard-Sullivan Theorem [C-G], [Mil-1] is generalized [Ebe] to the case $d \geq 2$ as follows: the multiplier map $\lambda_{\mathcal{H}}: \mathcal{H} \rightarrow \mathbb{D}$ is well defined for every $\mathcal{H}$, and it is an analytic $(d-1)$-to-one mapping which extends continuously to the boundary.

For each angle $\left.{ }^{1}\right) \phi \in \mathbb{R} / \mathbb{Z}$, the $d-1$ preimages under $\lambda_{\mathcal{H}}$ of the rays $\left\{r e^{i 2 \pi \phi}: 0<r \leq 1\right\}$ are called the internal rays of $\mathcal{H}$ with arguments $(\phi+n) /(d-1), n=0, \ldots, d-2$. In particular, the internal rays mapped by $\lambda_{\mathcal{H}}$ to the positive real axis (that is, $\phi=0$ ) divide the hyperbolic component $\mathcal{H}$ into $d-1$ sectors.

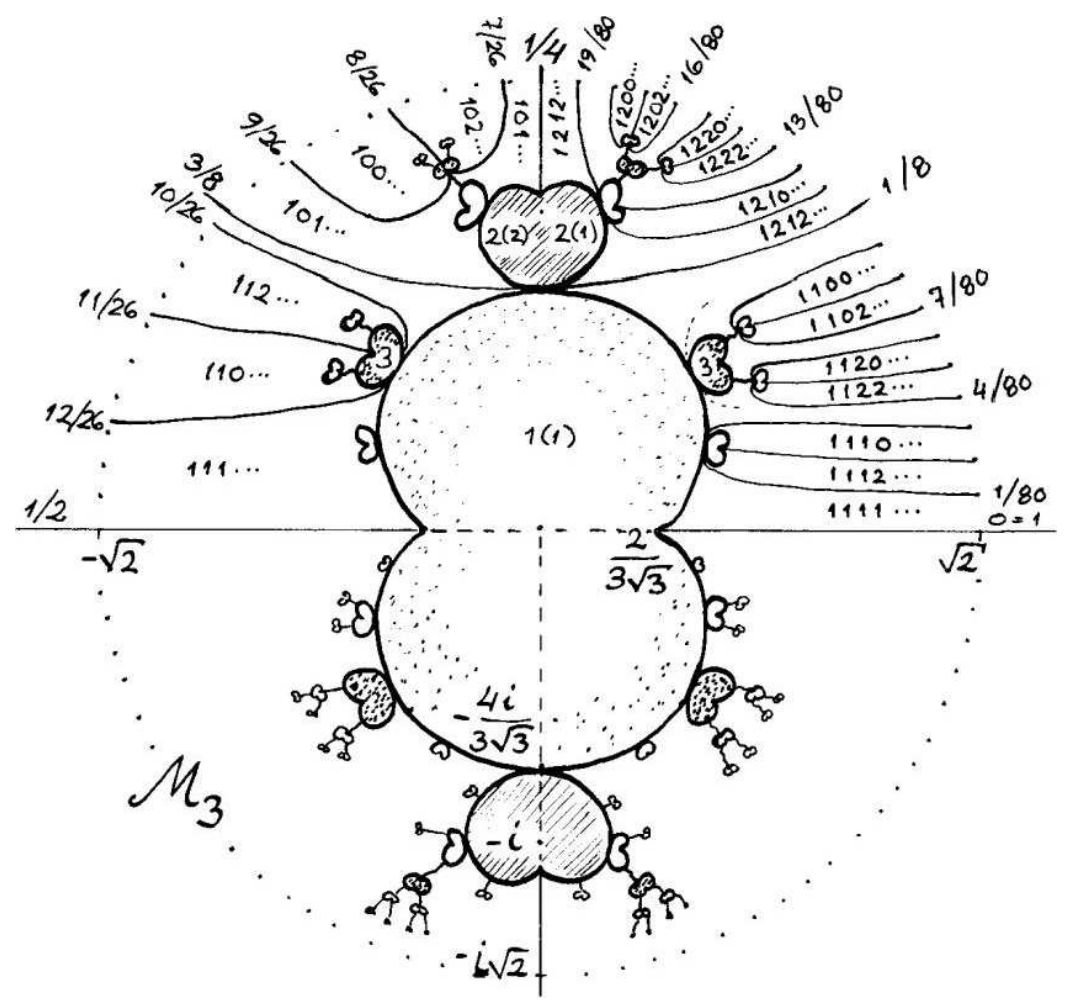

Fig. B

$\left({ }^{1}\right)$ Throughout this paper, all angles are measured in full turns (not radians or degrees). 
Polynomials $P_{c}$ with $c \in \partial \mathcal{H}$ at the limit points of these $d-1$ sectordividing rays thus have a parabolic orbit with multiplier $\lambda_{\mathcal{H}}(c)=1$. They are called the root and co-roots of $\mathcal{H}$ (see 2.8 below). The branching point $\lambda_{\mathcal{H}}^{-1}(0)$ is the centerpoint of $\mathcal{H}$.

The boundary of each hyperbolic sector also contains parameters $c=$ $\lambda_{\mathcal{H}}^{-1}\left(e^{i 2 \pi p / q}\right)$ with any rational number $\left.p / q \in\right] 0,1[$. Any neighborhood of such a point $c$ contains parameters $\widetilde{c}$ whose polynomials have attracting cycles with period $q\langle\mathcal{H}\rangle$. Hence $\widetilde{c}$ belongs to another hyperbolic component $\mathcal{K}$ whose root point is $c$. Then $\mathcal{K}$ is the $p / q$-satellite of $\mathcal{H}$.

Hyperbolic components whose roots do not belong to the boundary of another sector are called primitive components. Figure B shows all hyperbolic components with periods less than five in $\mathcal{M}^{3}$. Notice the satellites of the two sectors of the main component with internal angles $1 / 4,1 / 3,1 / 2,2 / 3,3 / 4$. There are also four primitive components with period three, each connected to a four-periodic satellite of a two-periodic sector by a "thread".

1.3. The main epicycloid. As an example, we consider the simplest special case $k=1$. There is a homeomorphism $\gamma$ between the closure of the hyperbolic component $\mathcal{H}=\mathcal{H}^{d}(1)$ and the set of corresponding attracting fixed points, which is a disk.

If $x \in \mathbb{C}$ is the fixed point of the polynomial $P_{c}: z \mapsto z^{d}+c$, then $c=x-x^{d}$. Writing $x=|x| e^{i 2 \pi \phi}$, we get

$$
\gamma(|x|, \phi)=|x| e^{i 2 \pi \phi}-|x|^{d} e^{i 2 \pi \phi d} .
$$

Fixing one of the parameters (angle $\phi$ or modulus $|x|$ ) yields a family of curves, $\left.\gamma_{\phi}:\right] 0, \infty\left[\rightarrow \mathbb{C}\right.$ or $\gamma_{|x|}: \mathbb{R} / \mathbb{Z} \rightarrow \mathbb{C}$ respectively. Now, the arc $\left.\gamma_{\phi}\right|_{0,1[}$ is an internal ray for each angle $\phi$.

The orbit $\{x\}$ has multiplier $\lambda=P_{c}^{\prime}(x)=d x^{d-1}$, so $|\lambda|=d|x|^{d-1}$, and $x \mapsto \lambda$ is an analytic $(d-1)$-to-one mapping. For a fixed modulus $|x|=a$, (1.4) gives a classical cycloidal curve [L-S-2] called an epitrokhoid [Kah]; $\gamma_{a}$ is drawn by a point $p$ on a circle with radius $b=a^{d}$ spinning at constant angular velocity $d \cdot v$ while its centerpoint is moving at angular velocity $v$ along a circle of radius $a=|x|$ centered at the origin. This curve intersects itself exactly if the point $p$ changes direction on its orbit seen from the origin $\left({ }^{2}\right)$, that is, if

$$
a v-b d v<0 \Leftrightarrow d>\frac{a}{b}=|x|^{1-d}=\frac{d}{|\lambda|} \Leftrightarrow|\lambda|>1 .
$$

The mapping $x \mapsto c$ is thus a homeomorphism when $|\lambda| \leq 1$, or $|x| \leq$ $d^{-1 /(d-1)}$. In other words, the disk of attracting and neutral fixed points $x$

$\left(^{2}\right)$ This is why epitrokhoids were used as a model of planetary motions before Kepler's invention of elliptic orbits; viewed from the Earth, e.g. Mars seems to make backward loops against the background stars. 
maps homeomorphically to the closure of the hyperbolic component. In the limiting case, $|x|=d^{-1 /(d-1)}$, the velocity $(a-b d) v$ of the point $p$ is zero when $p$ is nearest to the origin, so the curve $\gamma_{|x|}(\phi)$ has a sharp cusp inwards when $(d-1) \phi=0 \bmod 1$. This curve is an epicycloid, and the cusp points are the root and the co-roots.

1.5. Remark. For every $d$, the symmetry group of $\mathcal{M}^{d}$ is the dihedral group $D_{d-1}$. That the Mandelbrot set is symmetric with respect to reflection and rotation by an angle $1 /(d-1)$, follows from the fact that the polynomials $P_{\bar{c}}$ and $P_{\varrho c}$ (where $\varrho=e^{i 2 \pi /(d-1)}$ ) have dynamics similar to that of $P_{c}$. For details and the converse statement (there are no other symmetries), see [L-S-2] or [S-S].

To finish the first section, we prove another "folklore" result concerning the size of the Mandelbrot set of degree $d \geq 2$ :

1.6. Proposition. (1) $c \in \mathcal{M}^{d} \Rightarrow|c| \leq 2^{1 /(d-1)}$.

(2) $c \in \mathcal{M}^{d} \Leftrightarrow\left|P_{c}^{n}(0)\right| \leq 2^{1 /(d-1)} \forall n \in \mathbb{N}$.

(3) The bound $2^{1 /(d-1)}$ for $\mathcal{M}^{d}$ is sharp for even values of $d$ but not for odd ones.

Proof. (1) Denote by $\left(z_{n}\right)_{n \in \mathbb{N}}$ the iteration sequence such that $z_{0}=0$ and $z_{n}=z_{n-1}^{d}+c$. Take an arbitrary $\varepsilon>0$ and assume that $|c| \geq 2^{1 /(d-1)}+\varepsilon$; we show that then $c \notin \mathcal{M}^{d}$. The assumption first implies that

$$
\begin{aligned}
|c|^{d-1} & \geq 2+(d-1) 2^{(d-2) /(d-1)} \varepsilon+\ldots+\varepsilon^{d-1}>2+(d-1) \varepsilon, \\
\left|z_{2}\right| & =\left|c^{d}+c\right| \geq|c|\left(|c|^{d-1}-1\right)>|c|(1+(d-1) \varepsilon) .
\end{aligned}
$$

Assuming inductively that $\left|z_{n}\right|>|c|(1+(d-1) \varepsilon)^{n-1}$ for some $n \in \mathbb{N}$, we obtain

$$
\begin{aligned}
\left|z_{n+1}\right| & =\left|z_{n}^{d}+c\right| \geq\left|z_{n}\right|^{d}-|c|>|c|^{d}(1+(d-1) \varepsilon)^{(n-1) d}-|c| \\
& =|c|\left(|c|^{d-1}(1+(d-1) \varepsilon)^{(n-1) d}-1\right) \\
& >|c|\left((1+(d-1) \varepsilon)^{(n-1) d+1}+(1+(d-1) \varepsilon)^{(n-1) d}-1\right) \\
& >|c|(1+(d-1) \varepsilon)^{n} .
\end{aligned}
$$

Now $\left|z_{n}\right|>|c|(1+(d-1) \varepsilon)^{n-1}$ for all $n \in \mathbb{N}$, and $\left|z_{n}\right| \rightarrow \infty$ as $n \rightarrow \infty$. Because of (1.1), $|c|$ must be at most $2^{1 /(d-1)}$ if $c \in \mathcal{M}^{d}$, so we have proved the first statement.

(2) The right hand side condition is sufficient by definition. To show that it is also necessary, assume that $\left|z_{n}\right| \geq 2^{1 /(d-1)}+\varepsilon$ for some $c \in \mathcal{M}^{d}$ and $n \in \mathbb{N}$; by $(1),|c| \leq 2^{1 /(d-1)}$. Then

$$
\left|z_{n+1}\right|=\left|z_{n}^{d}+c\right| \geq\left|z_{n}\right|^{d}-|c|>2^{d /(d-1)}+d \varepsilon-2^{1 /(d-1)}=2^{1 /(d-1)}+d \varepsilon
$$

and hence

$$
\left|z_{n+k}\right|>2^{1 /(d-1)}+d^{k} \varepsilon \quad \forall k \in \mathbb{N},
$$


so the sequence is unbounded, which contradicts the assumption that $c \in$ $\mathcal{M}^{d}$

(3) If $c=-2^{1 /(d-1)}$, then 0 is mapped to a fixed point in two iterations:

$$
P_{c}: 0 \mapsto-2^{1 /(d-1)} \mapsto\left(-2^{1 /(d-1)}\right)^{d}-2^{1 /(d-1)}=2^{1 /(d-1)} \mapsto 2^{1 /(d-1)} .
$$

It follows that $-2^{1 /(d-1)} \in \mathcal{M}^{d}$. By dihedrality (1.5), also the points $2^{1 /(d-1)} e^{i \pi(1+2 k) /(d-1)}$ belong to $\mathcal{M}^{d}$ for every $k=0, \ldots, d-2$.

For odd values of $d$ however, this bound is not sharp. If $c=2^{1 /(d-1)} e^{i 2 \pi \varphi}$ for any $\varphi$, then

$$
P_{c}^{2}(0)=2^{1 /(d-1)} e^{i 2 \pi \varphi}\left(2 e^{i 2 \pi \varphi(d-1)}+1\right) .
$$

This number has absolute value greater than $2^{1 /(d-1)}$ unless $\varphi$ is an odd multiple of $1 / 2(d-1)$. But then $P_{c}^{2}(0)=-2^{1 /(d-1)} e^{i 2 \pi \varphi}$, so

$$
\left|P_{c}^{3}(0)\right|=\left|2^{1 /(d-1)} e^{i 2 \pi \varphi}\left(-2 e^{i 2 \pi \varphi(d-1)}+1\right)\right|=3 \cdot 2^{1 /(d-1)}>2^{1 /(d-1)} ;
$$

by (2), $c$ cannot be in $\mathcal{M}^{d}$.

(Since $\mathcal{M}^{d}$ is compact, there must be some number $\delta_{d}<2^{1 /(d-1)}$ such that $\mathcal{M}^{d} \subset \bar{B}\left(0, \delta_{d}\right)$ when $d$ is odd.) From 1.3 and 1.6 we conclude that

$$
\bar{B}\left(0,(d-1) d^{-d /(d-1)}\right) \subset \overline{\mathcal{H}}^{d}(1) \subset \mathcal{M}^{d} \subset \bar{B}\left(0,2^{1 /(d-1)}\right) .
$$

Since the radii of both these bounding disks tend to 1 as $d$ tends to infinity, the main epicycloid becomes increasingly disk-like and dominating in comparison to the area of the whole set $\mathcal{M}^{d}$.

\section{RAYS, ORBIT PORTRAITS, AND KNEADING SEQUENCES}

This section presents some important combinatorial tools that have been used for determining the structure of the Mandelbrot sets from the dynamics of the polynomials with different parameters.

2.1. External rays. The (dynamical) external ray with angle $\theta$ of the filled Julia set $K_{c}$ of a polynomial $P_{c}: z \mapsto z^{d}+c$, denoted by $\mathcal{R}_{\theta}^{c}$, is the preimage of the radial line $\left\{r e^{i 2 \pi \theta}: r>1\right\}$ under the conformal Böttcher map from $\widehat{\mathbb{C}} \backslash K_{c}$ to the exterior of the closed unit disk; consult [C-G] or [Bea] for details. The parameter rays $\mathcal{R}_{\theta}^{\mathcal{M}^{d}}$ are defined similarly to preimages of straight rays under the conformal mapping obtained by evaluating the Böttcher map at $c$ itself. If an external ray $\mathcal{R}_{\theta}$ lands (that is, has a limit when $r \searrow 1$ ) at a point $z$, then $\theta$ is called the external angle of $z$.

2.2. Periodic angles. The combinatorial approach to $\mathcal{M}^{d}$ is based on the fact that the polynomial $P_{c}$ maps each dynamical ray to another ray multiplying its argument by $d$. An angle $\theta$ in the circle $\mathbb{R} / \mathbb{Z}$ is $k$-periodic under the $d$-tupling map $\sigma_{d}: \theta \mapsto d \theta \bmod 1$ if and only if it is rational and 
of the form $\theta=t /\left(d^{k}-1\right)$ for some integers $t$ and (minimal) $k$. For example (see Figure C),

$$
\sigma_{3}: \frac{3}{11} \mapsto \frac{9}{11} \mapsto\left(\frac{27}{11}=\right) \frac{5}{11} \mapsto \frac{4}{11} \mapsto \frac{1}{11} \mapsto \frac{3}{11},
$$

so these angles are five-periodic under tripling; note that $3 / 11=66 /\left(3^{5}-1\right)$.
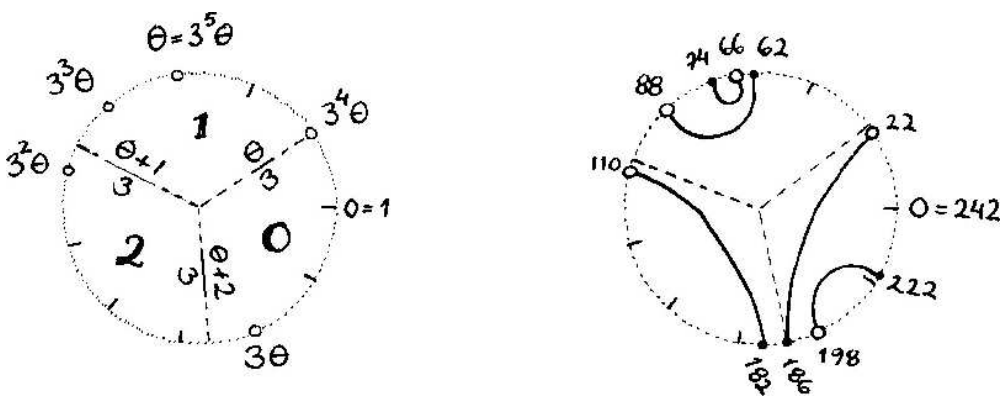

Fig. C

2.3. Orbit portraits. This theory was developed by Milnor; see [Mil-2] for details and proofs in the quadratic case and [Ebe], [Rie] for generalizations to the case $d \geq 2$.

If some dynamic rays with rational angles land at a parabolic or repelling $k$-periodic orbit $\left\{z_{1}, \ldots, z_{k}\right\}$ where $z_{j+1}=z_{j}^{d}+c$, and $A_{j}$ consists of all external angles of the point $z_{j}$ for each $j \in \mathbb{Z}_{k}$, then the following hold:

(1) Every $A_{j}$ has the same finite number, $v$, of angles.

(2) The $d$-tupling modulo 1 map $\sigma_{d}$ takes $A_{j}$ to $A_{j+1}$ bijectively, preserving the cyclic order of the angles.

(3) Every angle in $A_{1} \cup \ldots \cup A_{k}$ is periodic with the same period, $q k$ with some $q \in \mathbb{N}$.

(4) The sets $A_{j}$ are pairwise unlinked (i.e., are contained in disjoint intervals of the circle).

The set $\Theta=\left\{A_{1}, \ldots, A_{k}\right\}$ is then called the orbit portrait of the orbit in question. Moreover, every set satisfying the conditions (1)-(4) above occurs as the portrait of an orbit of some polynomials $P_{c}$.

An orbit portrait is essential if $v \geq 2$ or if $\Theta=\{\{0\}\}$. A portrait is primitive if $q=1$ and satellite otherwise. Satellite portraits always have just one cycle of angles (so $v=q \geq 2$ ), whereas essential primitive portraits have two different cycles (so $v=2$ ).

The connected components of each $\mathbb{R} / \mathbb{Z} \backslash A_{j}$ are called the complementary intervals. The characteristic interval $] \theta_{-}, \theta_{+}[$of $\Theta$ is the unique shortest one of them. 
The map $\sigma^{k}$ permutes the angles $\left\{\theta_{1}, \ldots, \theta_{q}\right\}\left(0<\theta_{1}<\ldots<\theta_{q}<1\right)$ in each $A_{j}$ so that for all $i \in \mathbb{Z}_{q}, \sigma^{k}\left(\theta_{i}\right)=\theta_{i+p}$ for some $p \in \mathbb{Z}_{q}$. The rotation number $p / q \in \mathbb{Q} / \mathbb{Z}$ of $\Theta$ is zero for primitive orbit portraits, and $0<p / q<1$ for satellite ones.

Figure $\mathrm{C}$ shows a primitive orbit portrait with characteristic interval ]$\frac{66}{242}, \frac{74}{242}[$, degree $d=3$, orbit period $k=5, q=1, v=2$.

2.4. Kneading sequences of angles. The kneading sequence of an angle $\theta \in \mathbb{R} / \mathbb{Z}$,

$$
K_{d}(\theta)=a_{1} a_{2} \ldots \in\{\mathbf{0}, \mathbf{1}, \ldots, \mathbf{d}-\mathbf{1}, \mathbf{\mathbf { 0 }}, \mathbf{\mathbf { 1 }}, \ldots, \underset{\mathbf{d}-\mathbf{1}}{\mathbf{0}}\}^{\mathbb{N}},
$$

is defined (according to [Ate], [B-K], [L-S-1], [Pen] etc.) as follows: The $d$ preimages $\frac{\theta+j}{d}\left(j \in \mathbb{Z}_{d}\right)$ of $\theta$ under the $d$-tupling map $\sigma_{d}$ (see 2.2) divide the circle into $d$ sectors of equal size. One of them contains $0=1$ and is labeled $\mathbf{0}$, the others are labeled counter-clockwise $\mathbf{1}$ through $\mathbf{d}-\mathbf{1}$. We list the labels of sectors where iterates of $\theta$ go to:

$$
K_{d}(\theta)_{n}:= \begin{cases}\mathbf{j} & \text { if } \left.\sigma_{d}^{n-1}(\theta) \in V_{j}^{\theta}:=\right] \frac{\theta+j-1}{d}, \frac{\theta+j}{d}[, \\ \mathbf{j}+\mathbf{1} & \text { if } \sigma_{d}^{n-1}(\theta)=\frac{\theta+j}{d} .\end{cases}
$$

In the previous example $(2.2$ and Figure $\mathrm{C}), K_{3}(3 / 11)=\overline{\mathbf{1 0 2 1} \mathbf{1}}$. Kneading sequences never start with $\mathbf{0}$, because $\theta / d<\theta<(\theta+d-1) / d$ for all $0<\theta<1$. The following result is a generalization of the quadratic case [L-S-1, 3.3], [Kau, 3.4]. The idea is shown in Figure D.

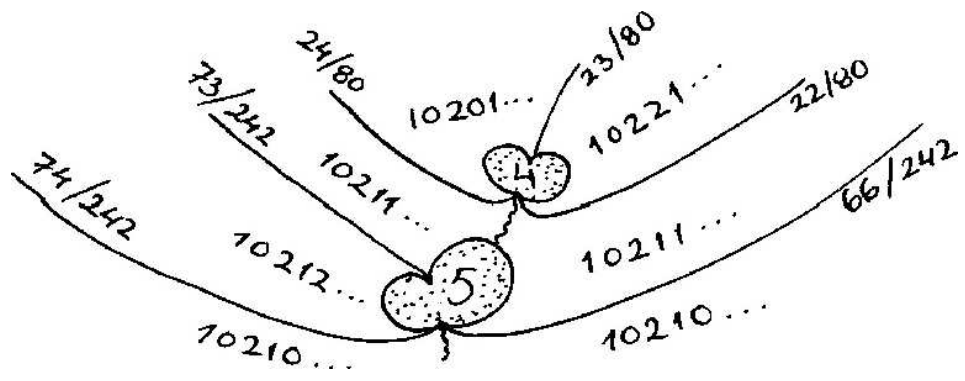

Fig. D

2.6. LEMMA. When the angle $\theta$ moves counter-clockwise around the circle, the nth entry in its kneading sequence changes from $j$ to $j+1$ precisely when $\theta$ crosses a rational angle of the form $(r d+j) /\left(d^{n}-1\right)$.

Proof. The digit $K_{d}(\theta)_{n}$ must change exactly at such angles, because by definition, 


$$
\begin{aligned}
K_{d}(\theta)_{n}=\underset{\mathbf{j}}{\mathbf{j}+\mathbf{1}} & \Leftrightarrow d^{n-1}(\theta)=\frac{\theta+j}{d} \bmod 1 \Leftrightarrow d^{n} \theta=\theta+j \bmod d \\
& \Leftrightarrow d^{n} \theta-\theta=r d+j \Leftrightarrow \theta=\frac{r d+j}{d^{n}-1}
\end{aligned}
$$

for any $r \in\left\{0,1, \ldots, d^{n-2}\right\}$. Whenever $0<\varepsilon<1 /\left(d^{n}-1\right)$,

$$
\sigma_{d}^{n-1}(\theta+\varepsilon)=\frac{\theta+j}{d}+\frac{d^{n} \varepsilon}{d}=\frac{(\theta+\varepsilon)+j}{d}+\frac{\left(d^{n}-1\right) \varepsilon}{d} \in V_{j+1}^{\theta+\varepsilon}
$$

(because the latter term is strictly between 0 and $1 / d)$, so $K_{d}(\theta+\varepsilon)_{n}=\mathbf{j}+\mathbf{1}$. Similarly, $K_{d}(\theta-\varepsilon)_{n}=\mathbf{j}$.

The two limit sequences

$$
K_{d}^{ \pm}(\theta):=\lim _{\varepsilon \rightarrow 0} K_{d}(\theta \pm \varepsilon) \in\{\mathbf{0}, \mathbf{1}, \ldots, \mathbf{d}-\mathbf{1}\}^{\mathbb{N}}
$$

exist for every $\theta$. For nonperiodic angles they are equal; if $\theta$ is $k$-periodic, they differ exactly at indices $n k$ for every $n \in \mathbb{N}$ so that $K_{d}^{+}(\theta)_{n k}=$ $K_{d}^{-}(\theta)_{n k}+1$. Periodic angles have periodic limit sequences, but an $n$-periodic sequence may belong to an angle whose period is a multiple of $n$ or even to a nonperiodic angle.

Orbit portraits and kneading sequences are used to prove the pattern in which the external rays land at the boundary of $\mathcal{M}^{d}$ (see again Figure B):

2.8. Structure Theorems ([D-H], [Mil-2], [Sch-2], [Ebe]). (1) Every parameter ray with periodic angle $\theta$ lands at a parameter $\widehat{c} \in \partial \mathcal{M}^{d}$. The polynomial $P_{\widehat{c}}$ has a parabolic orbit with multiplier $\lambda(\widehat{c})=1$ and portrait $\Theta$ containing $\theta$.

(2) Every parabolic parameter $\widehat{c}$ belongs to the boundary of some hyperbolic component $\mathcal{H}$.

(3) If the orbit portrait $\Theta$ is nonessential, then $\widehat{c}$ is a co-root of $\mathcal{H}$ and the landing point of exactly one external ray.

(4) If $\Theta$ is essential, then $\widehat{c}$ is the root of $\mathcal{H}$ and the landing point of exactly two external rays, $\mathcal{R}_{\theta_{-}}$and $\mathcal{R}_{\theta_{+}}$. The arguments of these rays are the two characteristic angles of $\Theta$.

(5) Every hyperbolic component has one root and $d-2$ co-roots.

(6) The two parameter rays $\mathcal{R}_{\theta_{-}}$and $\mathcal{R}_{\theta_{+}}$landing at $\widehat{c}$ bound the wake $W(\Theta)$ away from the origin. A polynomial $P_{c}$ has a repelling orbit with portrait $\Theta$ if and only if $c \in W(\Theta)$.

The main epicycloid is exceptional in that there is actually just one external ray landing at its rootpoint. However, the statements (4) and (6) are correct because the ray $\mathcal{R}_{0}=\mathcal{R}_{1}$ is "counted twice": the corresponding exceptional essential orbit portrait $\Theta=\{\{0\}\}$ has characteristic interval ] $0,1[$. 
It follows that the unions of external and internal rays landing at the root and co-roots of each hyperbolic component with period $k \geq 2$ divide the parameter plane into $d$ regions. One of them contains the origin, the $d-1$ others are called the wakes of the respective hyperbolic sectors. In the case of the main epicycloid, the plane just gets divided into the $d-1$ sector wakes.

\section{VISIBLE TREES}

The Structure Theorems imply that all parameters in each Mandelbrot set sit in wakes that are either nested or disjoint; this feature gives rise to a treelike structure.

3.1. Partial order. The landing feature of parameter rays described in 2.8 determines a partial ordering in the Mandelbrot set: each parameter $c \in$ $\mathcal{M}^{d}$ belongs to a certain set $U(c)$ of nested wakes of hyperbolic components and sectors. Another parameter $c^{\prime}$ is above $c$, denoted $c^{\prime} \succ c$, if $U(c) \subset U\left(c^{\prime}\right)$. Then $c$ is below $c^{\prime}$, denoted $c \prec c^{\prime}$.

Belonging to the same set of wakes is obviously an equivalence relation, so we define $c^{\prime} \sim c$ if $U\left(c^{\prime}\right)=U(c)$. In particular, all parameters in the closure of a hyperbolic component except the root are equivalent, so we can also write $\mathcal{A} \prec \mathcal{B}$ if $\mathcal{A}$ and $\mathcal{B}$ are hyperbolic components or sectors and $\mathcal{B}$ is in the wake of $\mathcal{A}$ (so, $\mathcal{A} \prec \mathcal{B} \Leftrightarrow W(\mathcal{A}) \supset W(\mathcal{B})$; note that the set $U(c)$ is not to be confused with the wake $W(c)$ ). From now on, we will usually talk in terms of equivalence classes.

This definition distinguishes a hyperbolic component from its root, in contrast to the combinatorial class [Kel, 1.14]. The set of all accumulation points of a parameter ray with irrational angle, together with any "weird" components that may be bounded by that set, would also be one equivalence class, so we need not worry whether the Mandelbrot set is locally connected or not.

3.2. Combinatorial arc, narrowness and visibility. The collection of all sectors $\mathcal{B}$ such that $\mathcal{C} \prec \mathcal{B} \prec \mathcal{A}$ is called the combinatorial arc $] \mathcal{C}, \mathcal{A}[$. We write $[\mathcal{C}, \mathcal{A}[,] \mathcal{C}, \mathcal{A}],[\mathcal{C}, \mathcal{A}]$ if one or both ends are included.

A hyperbolic sector $\mathcal{C}$ is narrow if there are no components above it with period $\langle\mathcal{C}\rangle$ or less; i.e., $\langle\mathcal{A}\rangle>\langle\mathcal{C}\rangle$ for all $\mathcal{A} \succ \mathcal{C}$. (Equivalently, the wake of $\mathcal{C}$ has width $1 /\left(d^{\langle\mathcal{C}\rangle}-1\right)$; see $\S 7$. $)$

A hyperbolic component $\mathcal{A}$ above a sector $\mathcal{C}$ is visible from $\mathcal{C}$ if there are no sectors on the combinatorial arc between them with periods $\langle\mathcal{A}\rangle$ or less; i.e., $\langle\mathcal{B}\rangle>\langle\mathcal{A}\rangle$ for all $\mathcal{B} \in] \mathcal{C}, \mathcal{A}[$.

For example, in Figure B, the four-periodic component with external angles $\frac{4}{80}, \frac{5}{80}, \frac{6}{80}$ is not visible from the main epicycloid because the threeperiodic sector with external angles $\frac{1}{26}, \frac{2}{26}$ is "blocking the view". The four- 
periodic sector with external angles $\frac{12}{80}, \frac{19}{80}$ is not narrow because the wake contains a three-periodic component with external angles $\frac{4}{26}, \frac{5}{26}, \frac{6}{26}$.

3.3. Lavaurs's Lemma ([Lav], [L-S-1, 3.8]). If $\mathcal{A} \prec \mathcal{C}$ and $\langle\mathcal{A}\rangle=\langle\mathcal{C}\rangle=k$, then there is a hyperbolic component $\mathcal{B}$ such that $\mathcal{A} \prec \mathcal{B} \prec \mathcal{C}$ and $\langle\mathcal{B}\rangle<k$.

3.4. Trees. The set of hyperbolic components visible from a base sector $\mathcal{C}$ with period $k$ are arranged in visible trees: every satellite component is visible from its parent sector but invisible from all other components, so each visible tree of $\mathcal{C}$ consists of a satellite component, stem, and a finite number of primitive components. (Satellite and primitive components were defined in 1.2.) The tree stemming from the $p / q$-satellite of $\mathcal{C}$ is denoted by $\mathcal{T}_{p / q}(\mathcal{C})$; all components in it must have periods at most $q k$.

Components $\mathcal{B} \in \mathcal{T}_{p / q}(\mathcal{C})$ that have nothing visible (from $\mathcal{C}$ ) above them are the tips of their branches. They are all narrow. The component $\mathcal{A}$ with the smallest period of all in the tree is called the treetop. The arc $] \mathcal{C}, \mathcal{A}]$ between the stem and the treetop is the primary trunk of $\mathcal{T}_{p / q}(\mathcal{C})$.
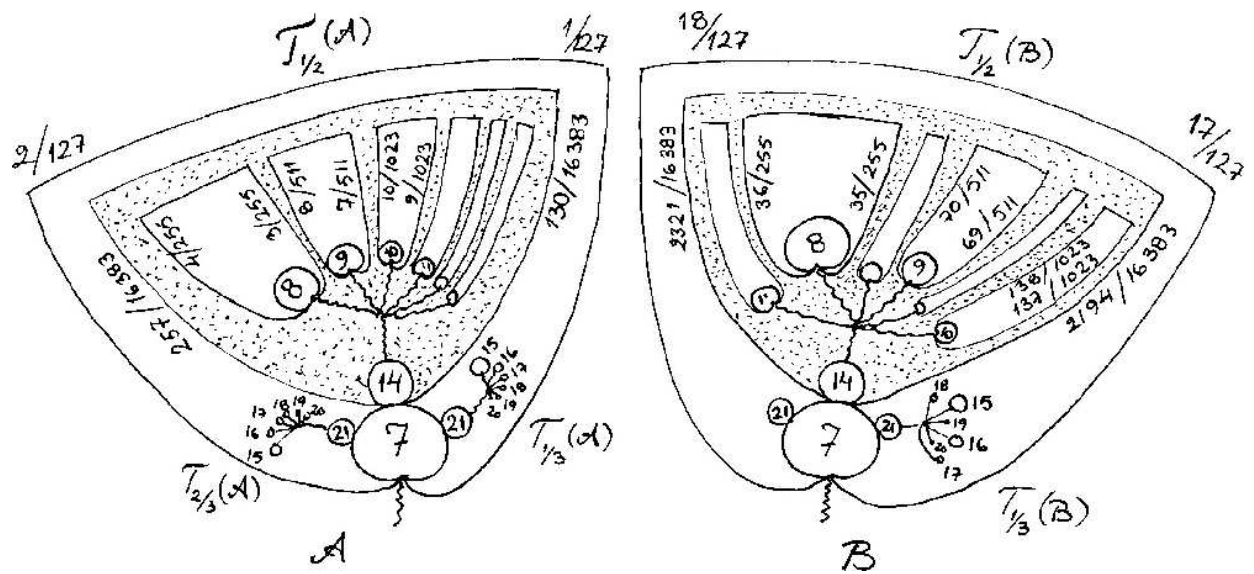

Fig. E

For example, Figure E shows two base components with period seven, their satellites at internal angles $1 / 3,1 / 2,2 / 3$, and six primitive components above each satellite that are visible from the base.

3.5. Equivalence of trees. Two trees $\mathcal{T}$ and $\mathcal{T}^{\prime}$ are called combinatorially equivalent, denoted by $\mathcal{T} \sim_{\mathrm{c}} \mathcal{T}^{\prime}$, if there is a homeomorphism between them which adds a constant $s \in \mathbb{Z}$ to the period of each component, i.e., maps each component with period $n$ in $\mathcal{T}$ into a component with period $n+s$ in $\mathcal{T}^{\prime}$. If $\mathcal{T} \sim_{\mathrm{c}} \mathcal{T}^{\prime}$ and the trees stem from the same base sector $\mathcal{C}$ at internal angles $p_{1} / q_{1}, p_{2} / q_{2}$ respectively, then the satellites must map into each other, so the constant $s$ equals $\left(q_{2}-q_{1}\right) k$ where $k=\langle\mathcal{C}\rangle$. 
The external rays, together with the natural order of angles on the circle, determine the embedding of the Mandelbrot set into the parameter plane. If a homeomorphism between the trees preserves their embeddings into the plane (either preserving or reversing orientation), we say that the trees are topologically equivalent: $\mathcal{T} \sim_{\mathrm{t}} \mathcal{T}^{\prime}$. Neither condition implies the other, but if the two trees are both topologically and combinatorially equivalent, we write $\mathcal{T} \approx \mathcal{T}^{\prime}$.

For example, the trees $\mathcal{T}_{1 / 2}(\mathcal{A})$ and $\mathcal{T}_{1 / 2}(\mathcal{B})$ in Figure E are combinatorially equivalent but not topologically (both trees have a bunch of six primitive components with equal periods respectively, but the branches are in different order). In addition, $\mathcal{T}_{1 / 3}(\mathcal{A}) \approx \mathcal{T}_{2 / 3}(\mathcal{A}) \approx \mathcal{T}_{1 / 2}(\mathcal{A})$, with an orientation-preserving homeomorphism (the periods of the components increase clockwise in both trees). Similarly, $\mathcal{T}_{1 / 2}(\mathcal{B}) \approx \mathcal{T}_{1 / 3}(\mathcal{B})$.
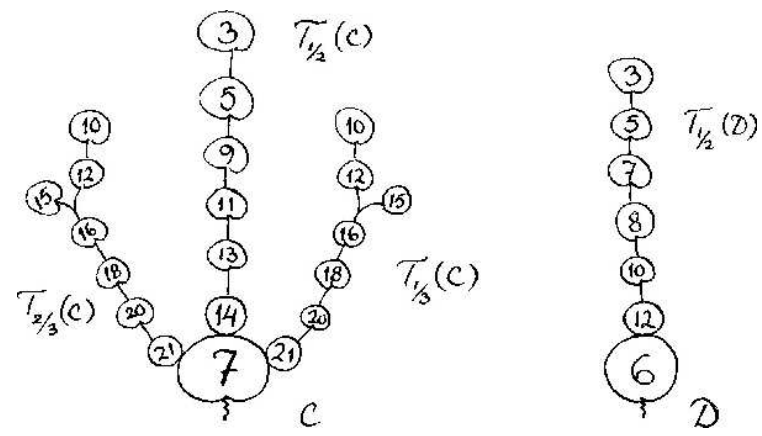

Fig. F

In Figure $\mathrm{F}, \mathcal{T}_{1 / 3}(\mathcal{C}) \approx \mathcal{T}_{2 / 3}(\mathcal{C})$ with an orientation-reversing homeomorphism (the 15-periodic components in the two trees branch off the primary trunks to the right and left, respectively). These trees are not equivalent to $\mathcal{T}_{1 / 2}(\mathcal{C})$ (neither topologically nor combinatorially: it lacks a component with period $15-7=8$ altogether). Finally, the tree $\mathcal{T}_{1 / 2}(\mathcal{C})$ is topologically equivalent to $\mathcal{T}_{1 / 2}(\mathcal{D})$ but not combinatorially (both consist of six components in one chain, but the periods of the corresponding ones do not differ by a constant number).

In this work we are more interested in combinatorial equivalence than topological, because it is relevant in terms of symbolic dynamics even for nonexistent trees $(\S 5)$.

3.6. Translation Principle. Two trees $\mathcal{T}_{p_{1} / q_{1}}$ and $\mathcal{T}_{p_{2} / q_{2}}$ of a base sector $\mathcal{C}$ are combinatorially equivalent for any $p_{1}, p_{2}, q_{1}$, and $q_{2}$.

This statement is true for narrow base sectors; the proof in [L-S-1, 10.2] for the quadratic case $d=2$ is generalized here for $d>2$ in 8.2. It also holds in many other cases, but it is not true in general for visible trees of 
hyperbolic sectors, as shown, e.g., in [Kau, §4]. Another counter-example is shown in Figure F. However, Translation Principle does hold in general for formal trees, which we will prove in 6.14 . The following weaker statement, Partial Translation Principle, is true in general at least in the quadratic case, as proved in [Kel, 3.78].

3.7. TheOREM. Let $\mathcal{C}$ be any hyperbolic sector in $\mathcal{M}^{2}$. Then all its visible trees $\mathcal{T}_{p / q}$, except perhaps $\mathcal{T}_{1 / 2}$, are topologically equivalent to $\mathcal{T}_{1 / 3}$.

Theorem 8.12 in this paper is related to 3.7: a certain class of "nonexistent components" (cf. 5.1) can only be missing in the tree at internal angle $1 / 2$.

\section{INTERNAL ADDRESSES}

In order to reach a certain parameter (e.g., the landing point of a given external ray) from the main epicycloid, one must walk through a certain sequence of hyperbolic sectors. By listing the periods and sector numbers of some of them we obtain a sequence called the internal address. These are introduced and studied in [L-S-1] using kneading sequences of the external angles.

4.1. Kneading sequences of parameter classes. We first extend the definition of kneading sequence from angles to parameters in the Mandelbrot set (or actually, to parameter classes in $\mathcal{M}^{d} / \sim$; cf. 3.1). Every parameter in $\partial \mathcal{M}^{d}$ is an accumulation point of some external rays, so each parameter class $c \in \mathcal{M}^{d} / \sim$ has some external angles. If they are nonperiodic, the angles all have the same kneading sequence in $\{\mathbf{0}, \mathbf{1}, \ldots, \mathbf{d}-\mathbf{1}\}^{\mathbb{N}}$ (by Lemma 2.6). This sequence is defined as $K(c)$.

Parameters with periodic external angles can also be given kneading sequences. By the Structure Theorem 2.8, every hyperbolic component $\mathcal{H}$ has two rays landing at its root and $d-2$ at its co-roots. We name the angles $\theta_{1}, \ldots, \theta_{d}$ in the natural order, starting and ending at the root point $\widehat{c}$. These $d$ angles all have the same period $k$ under the $d$-tupling map $\sigma_{d}$.

Furthermore, $K_{d}^{+}(\theta)=K_{d}^{-}(\varphi)$ for any two angles $\theta, \varphi$ with equal periods whose parameter rays are not separated by any wake boundary (2.6). This is the case for each pair of rays bounding the wake of a sector $\mathcal{H}^{s}$ of $\mathcal{H}$. We call the angles $\theta_{s}, \theta_{s+1}$ of these rays the external arguments of $\mathcal{H}^{s}$, and

$$
K_{d}^{+}\left(\theta_{s}\right)=K_{d}^{-}\left(\theta_{s+1}\right)=: K_{d}\left(\mathcal{H}^{s}\right)
$$

its kneading sequence (as in [L-S-1]). The symbol $K_{d}\left(\theta_{s}\right)_{n k}$ changes at each $\theta_{s}$, but since there are $d$ of them, it returns after one round; if $K_{d}\left(\theta_{1}\right)_{n k}=$ ${ }_{\mathbf{j}}^{\mathbf{j}+\mathbf{1}}$, then $K_{d}\left(\theta_{d}\right)_{n k}={ }_{\mathbf{j}-\mathbf{1}}^{\mathbf{j}}\left(j \in \mathbb{Z}_{d}\right)$. Therefore

$$
K_{d}^{-}\left(\theta_{1}\right)=K_{d}^{+}\left(\theta_{d}\right)=: K_{d}(\widehat{c})=: \widehat{K}_{d}(\mathcal{H}),
$$


and we call this the root sequence of the hyperbolic component $\mathcal{H}$. For example, the root sequence of the five-periodic component in $\mathcal{M}^{3}$ shown in Figure D is $\overline{\mathbf{1 0 2 1 0}}$ (whereas the kneading sequences for the two sectors of the component are $\overline{\mathbf{1 0 2 1 1}}$ and $\overline{\mathbf{1 0 2 1 2}}$ ).

4.4. Internal address. The rough internal address of any parameter $c \in \mathcal{M}^{d}$ is the sequence of integers

$$
n_{1} \longmapsto n_{2} \longmapsto \ldots
$$

defined as follows: $n_{1}=1, c_{1}=0$. Among all pairs of periodic parameter rays separating $c$ from $c_{j}$, exactly one pair has minimal period, $n_{j+1}$, and they land at a parameter $c_{j+1}$.

Lavaurs's Lemma [Lav] guarantees that $c_{j}$ is unique at each step, and that the sequence is strictly increasing. The point $c_{j}$ is obviously the root of some hyperbolic component $\mathcal{H}_{j}$, and $c$ sits in the wake of one of its sectors, $s_{j}$. Numbering the sectors of $\mathcal{H}_{j}$ from 1 through $d-1$ counter-clockwise from the root, as in 4.1, one gets the internal address of $c$,

$$
A(c):=n_{1}\left(s_{1}\right) \longmapsto n_{2}\left(s_{2}\right) \longmapsto \ldots
$$

4.7. Remark. All parameters in a hyperbolic sector share a finite internal address, so we can speak of internal addresses of sectors. Skipping the last sector number we get an address of the form $1\left(s_{1}\right) \longmapsto \ldots \longmapsto n_{j}\left(s_{j}\right) \longmapsto k$, which refers to the whole hyperbolic component and its boundary, except the rootpoint.

Primitive parabolic points $\widehat{c}$ have infinite internal addresses, and the rootpoint's address is also called the root address of the hyperbolic component. Bifurcation points (or roots of satellite components), on the other hand, share the finite address of the parent sector. All parameters not in closures of hyperbolic components have infinite addresses.

4.8. Address classes. Several parameter classes in different parts of $\mathcal{M}^{d} / \sim$ may share an internal address; for example, all the four five-periodic satellites of the main cardioid of $\mathcal{M}^{2}$ have internal address $1 \longmapsto 5$. Such components could be distinguished by the (rational) internal angles $p_{j} / q_{j}$ at which the combinatorial arc crosses each sector $H_{j}^{s_{j}}$ in the internal address; angled internal address determines a component uniquely, as shown in [L-S-1].

But the internal address turns out to determine the denominators $q_{j}$. Furthermore, if an internal address is realized by a hyperbolic sector with some sequence of numerators, it is also realized if one replaces any of the numerators $p_{j}$ with any other number $p_{j}^{\prime}$ less than and coprime to $q_{j}$. Hence, any internal address is either realized by any possible set of angles or not at all. 
To keep the notation simpler, we therefore consider just internal addresses without angles, and identify all parameters sharing one. This yields another equivalence relation, denoted by $\simeq$. Each element of the quotient space $\mathcal{M}^{d} / \simeq$ consists of one or several parameter classes in $\mathcal{M}^{d} / \sim$ (as defined in 3.1). (By the words "parameter", "component" etc. we will generally refer to elements of the space $\mathcal{M}^{d} / \simeq$.)

4.9. LSA. Given two parameter classes $\mathcal{C} \prec \mathcal{A}$, we can use Lemma 2.6 to find the minimal period of hyperbolic components on the combinatorial arc between them, by comparing their kneading sequences; it is the index $n$ at which $\widehat{K}_{d}(\mathcal{A})$ and $K_{d}(\mathcal{C})$ first differ. The kneading sequence of the $n$-periodic sector $\mathcal{B} \in] \mathcal{C}, \mathcal{A}$ [ is then obtained by repeating the first $n$ entries of $\widehat{K}_{d}(\mathcal{A})$.

Comparing iteratively $K_{d}(\mathcal{B})$ (or $\widehat{K}_{d}(\mathcal{B})$ ) to the original kneading sequences, one finds a sequence of hyperbolic sectors with increasing periods and nested wakes approaching $\mathcal{A}$ from below (or $\mathcal{C}$ from above, respectively). We call this procedure the Lau-Schleicher Algorithm (LSA) [L-S-1, 12.2]. In particular, any parameter (class) $\mathcal{A}$ is above some sector $\mathcal{C}^{s}$ of the main epicycloid, so comparing the kneading sequence of $\mathcal{A}$ to $K_{d}\left(\mathcal{C}^{s}\right)=\overline{\mathbf{s}}$, LSA yields its internal address.

4.10. Example. (See Figures B and D.) Comparing the root sequence

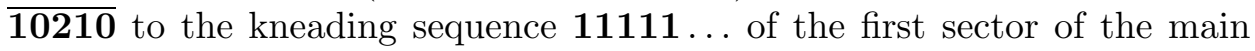
epicycloid, 1(1), one finds the first difference at digit $2=n_{2}: s_{2}=0-1$ $=3-1=2$. The original root sequence then differs from 101010 $\ldots$ first at $3=n_{3}$, and $s_{3}=2-1=1$. The first difference from $102102 \ldots$ occurs at $6>5$, so the internal address of this hyperbolic component is $1(1) \longmapsto$ $2(2) \longmapsto 3(1) \longmapsto 5$.

\section{FORMAL ADDRESSES AND FORMAL KNEADING SEQUENCES}

The Lau-Schleicher Algorithm actually works more generally: it is not necessary to assume a given sequence to occur as the kneading sequence or internal address of some hyperbolic sector. Therefore we can study the Mandelbrot set as a subspace of a more general symbol space.

5.1. Definitions.

- A $d$-kneading sequence is a sequence $\mathbf{a}=a_{1} a_{2} \ldots \in\{\mathbf{0}, \mathbf{1}, \ldots, \mathbf{d}-\mathbf{1}\}^{\mathbb{N}}$ with $a_{1} \neq \mathbf{0}$. We denote by $\Sigma^{d}$ the space of all such sequences equipped with the metric [Ate]

$$
|\mathbf{a}-\mathbf{b}|:=\sum_{i=1}^{\infty} \frac{\left|a_{i}-b_{i}\right|}{d^{i}} .
$$

- A rough address is an increasing sequence of integers starting with 1. 
- A (formal) $d$-address is a sequence $A=n_{1}\left(s_{1}\right) \longmapsto n_{2}\left(s_{2}\right) \longmapsto \ldots$, where $n_{1} \longmapsto n_{2} \longmapsto \ldots$ is a rough address and $s_{j} \in \mathbb{Z}_{d} \backslash\{0\}$ for all $j$. Denote the address space by $\Lambda^{d}$.

The metric (5.1a) will give a topological structure for the address space. Many formal addresses appear as the internal addresses of hyperbolic sectors or other parameter classes, but not all. Here, these nonrealizable addresses are thought to be inhabited by nonexistent parameters. Hence we may identify, e.g., a hyperbolic sector with its address, and study later whether it exists or not.

5.2. Addresses to kneading sequences and back. When used for turning a kneading sequence into an address, the Lau-Schleicher Algorithm can be written explicitly as follows:

Given a kneading sequence $\mathbf{a}:=a_{1} a_{2} \ldots \in \Sigma^{d}$, set $n_{1}=1$. For all $i \in \mathbb{N}$, $n_{i}$ is the first index at which the two sequences $\mathbf{a}$ and $\mathbf{b}=\overline{a_{1} \ldots a_{n_{(i-1)}}}$ differ; set $s_{i}:=b_{n_{i}}-a_{n_{i}}$. This yields a formal address $A=1\left(s_{1}\right) \longmapsto n_{2}\left(s_{2}\right) \longmapsto$ $\ldots \in \Lambda^{d}$.

The procedure is obviously reversible and independent of whether the given sequences actually occur in parameter classes of $\mathcal{M}^{d}$. Hence LSA defines a bijective mapping $\varkappa_{d}$ from the set $\Lambda^{d}$ of formal addresses to the space $\Sigma^{d}$ of kneading sequences:

$$
A=1\left(s_{1}\right) \longmapsto n_{2}\left(s_{2}\right) \longmapsto \ldots \in \Lambda^{d} \Rightarrow \varkappa_{d}(A):=\mathbf{a}=a_{1} a_{2} \ldots \in \Sigma^{d}
$$

such that

$$
\begin{aligned}
a_{1} \ldots a_{n_{2}-1} & =\mathbf{s}_{1} \ldots \mathbf{s}_{1}, \quad a_{n_{2}}=\mathbf{s}_{1}+\mathbf{s}_{2} ; \\
a_{1} \ldots a_{n_{j}-1} & =\left.\left(\overline{a_{1} \ldots a_{n_{(j-1)}}}\right)\right|_{n_{j}-1}, \\
a_{n_{j}} & =\left(\overline{a_{1} \ldots a_{n_{(j-1)}}}\right)_{n_{j}}+\mathbf{s}_{j} \quad \forall j .
\end{aligned}
$$

The bijection $\varkappa_{d}: \Lambda^{d} \rightarrow \Sigma^{d}$ induces a combinatorial and topological structure to the set $\Lambda^{d}$ from $\Sigma^{d}$. The space thus obtained contains the factor space $\mathcal{M}^{d} / \simeq$ (defined in 4.8) as a proper subset.

5.4. Finite addresses. Finite formal $d$-addresses $A=1\left(s_{1}\right) \longmapsto \ldots \longmapsto$ $n(s)$ ending with $n \in \mathbb{N}$ form a subspace $\Lambda_{n}^{d} \subset \Lambda^{d}$; we call $n=:|A|$ the length of the address $A$. They get mapped by $\varkappa_{d}$ to periodic kneading sequences with exact period $n$; the set $\dot{\Sigma}_{n}^{d}:=\varkappa_{d}\left(\Lambda_{n}^{d}\right)$ is a proper subset of $\Sigma_{n}^{d}:=\{\mathbf{a}$ : $\left.a_{i}=a_{i+n} \forall i \in \mathbb{N}\right\}$.

For each initial word $a_{1} \ldots a_{n-1}\left(a_{j} \in\{\mathbf{0}, \mathbf{1}, \ldots, \mathbf{d}-\mathbf{1}\}\right)$, exactly one choice for the next entry, $a_{n}$, does not stop LSA yielding the number $n$ to the address, so the remaining $d-1$ choices each do give an $n$-periodic sequence corresponding to an element of $\dot{\Sigma}_{n}^{d}$. The number of formal $d$-addresses of 
length $n$ is thus

$$
\# \dot{\Sigma}_{n}^{d}=\# \Lambda_{n}^{d}=d^{n-2}(d-1)^{2}
$$

$(d-1$ choices for $j=1$ and $j=n, d$ choices for $1<j<n)$. Not making the last choice means just referring to all the $d-1$ sectors of one hyperbolic component (whether this component exists or not). Hence we may call formal addresses of the type $A=1\left(s_{1}\right) \longmapsto \ldots \longmapsto n_{i}\left(s_{i}\right) \longmapsto n$ component addresses (in accordance with 4.8 ). The corresponding kneading sequence is denoted by replacing the last symbol in the periodic word by an asterisk: $\mathbf{a}=\overline{a_{1} \ldots a_{n-1} *}$.

The number of component addresses is thus $d^{n-2}(d-1)$. This number can be compared to the number of existing hyperbolic components with period $n$, which is $\left(d^{n}-2\right) / d$ if $n$ is prime and less otherwise: all the $d^{n}-2$ external rays with angle period $n$, or strictly dividing $n$, land at components with the same period in groups of $d$ by the Structure Theorems [Mil-2] [Ebe]. Taking into account that some addresses are realized by several components (cf. 4.8), one can estimate the number of nonexistent components in $\Lambda_{n}^{d}$ for each $d, n$.

5.5. ExAmple. The periodic words of elements of $\Sigma_{6}^{2}$ and the corresponding formal addresses are listed below. The first $2^{6-2} \cdot 1=16$ lines correspond to elements of $\dot{\Sigma}_{6}^{2}$. The first number shows how many six-periodic hyperbolic components have each one as its internal address. Their total number is $\left[\left(2^{6}-2\right)-\left(2^{3}-2\right)-\left(2^{2}-2\right)\right] / 2=27$.

$$
\begin{aligned}
& 2 \quad 111110 \quad 1 \longmapsto 6 \\
& 4 \quad 111100 \quad 1 \longmapsto 5 \longmapsto 6 \\
& 2 \quad 111010 \quad 1 \longmapsto 4 \longmapsto 6 \\
& 2 \quad 111000 \quad 1 \longmapsto 4 \longmapsto 5 \longmapsto 6 \\
& 2110111 \quad 1 \longmapsto 3 \longmapsto 6 \\
& 2 \quad 110100 \quad 1 \longmapsto 3 \longmapsto 5 \longmapsto 6 \\
& 2 \quad 110010 \quad 1 \longmapsto 3 \longmapsto 4 \longmapsto 6 \\
& 2 \quad 110000 \quad 1 \longmapsto 3 \longmapsto 4 \longmapsto 5 \longmapsto 6 \\
& 2101011 \quad 1 \longmapsto 2 \longmapsto 6 \\
& 2 \quad 101000 \quad 1 \longmapsto 2 \longmapsto 5 \longmapsto 6 \\
& 1 \quad 101111 \quad 1 \longmapsto 2 \longmapsto 4 \longmapsto 6 \\
& 0 \quad 101100 \quad 1 \longmapsto 2 \longmapsto 4 \longmapsto 5 \longmapsto 6 \\
& 1 \quad 100101 \quad 1 \longmapsto 2 \longmapsto 3 \longmapsto 6 \\
& 1 \quad 100110 \quad 1 \longmapsto 2 \longmapsto 3 \longmapsto 5 \longmapsto 6 \\
& 1 \quad 100011 \quad 1 \longmapsto 2 \longmapsto 3 \longmapsto 4 \longmapsto 6 \\
& 1 \quad 100000 \quad 1 \longmapsto 2 \longmapsto 3 \longmapsto 4 \succ 5 \longmapsto 6
\end{aligned}
$$




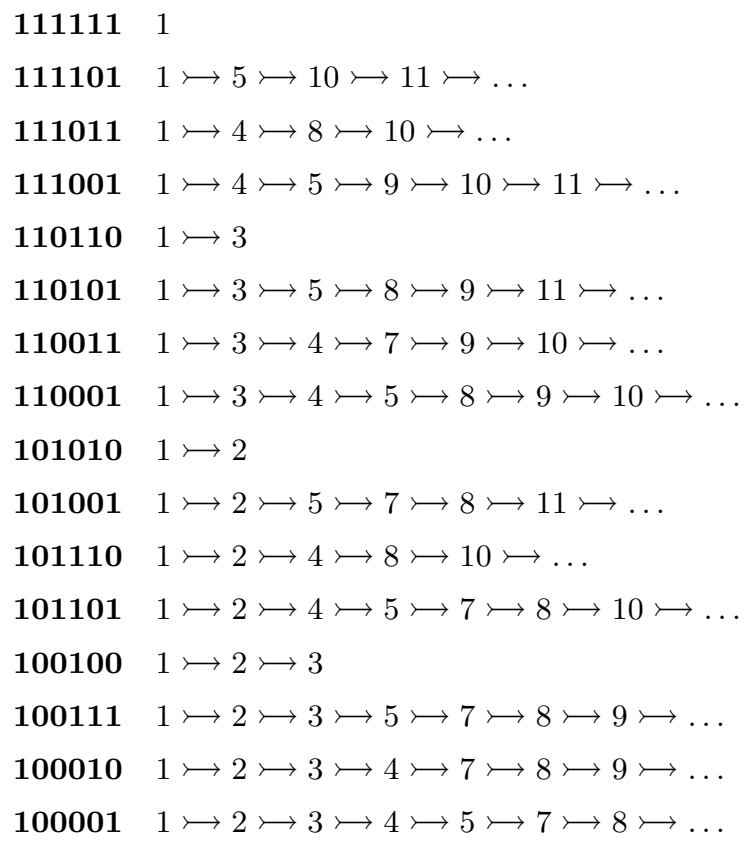

5.6. REMARK. For all $d$ and $n, \dot{\Sigma}_{n}^{d}$ is a proper subset of $\dot{\Sigma}_{n}^{d+1}$. Mapping a kneading sequence with $\varkappa_{d+1}$ naturally yields the same rough address as $\varkappa_{d}$, but the sector numbers may be different. For example, the kneading sequence $\mathbf{c}=\overline{\mathbf{1 0 0 1 1 0}} \in \dot{\Sigma}_{6}^{2}$ gets mapped as follows:

$$
\begin{aligned}
& \varkappa_{2}^{-1}(\mathbf{c})=1(1) \longmapsto 2(1) \longmapsto 3(1) \longmapsto 5(1) \longmapsto 6(1), \\
& \varkappa_{3}^{-1}(\mathbf{c})=1(1) \longmapsto 2(2) \longmapsto 3(2) \longmapsto 5(1) \longmapsto 6(2), \\
& \varkappa_{4}^{-1}(\mathbf{c})=1(1) \longmapsto 2(3) \longmapsto 3(3) \longmapsto 5(1) \longmapsto 6(3), \\
& \varkappa_{5}^{-1}(\mathbf{c})=1(1) \longmapsto 2(4) \longmapsto 3(4) \longmapsto 5(1) \longmapsto 6(4), \\
& \varkappa_{6}^{-1}(\mathbf{c})=1(1) \longmapsto 2(5) \longmapsto 3(5) \longmapsto 5(1) \longmapsto 6(5) .
\end{aligned}
$$

5.7. Root sequence. Let $B \in \Lambda_{n}^{d}$ be an address and $\varkappa_{d}(B)=\mathbf{b}=$ $\overline{b_{1} \ldots b_{n}} \in \dot{\Sigma}_{n}^{d}$ its kneading sequence. We define its root sequence $\widehat{\varkappa}_{d}(B):=$ $\widehat{\mathbf{b}}:=\overline{b_{1} \ldots b_{n}^{\prime}}$ by replacing $b_{n}$ with the one symbol $b_{n}^{\prime}$ such that $\widehat{\mathbf{b}} \notin \dot{\Sigma}_{n}^{d}$.

The root sequence $\widehat{\mathbf{b}}$ has the following alternatives. If it has period exactly $n$, then $\varkappa_{d}^{-1}(\widehat{\mathbf{b}})$ must be an infinite address skipping $n$; the set of such sequences is denoted by $\Sigma_{n, \infty}^{d}$. Unless $n$ is a prime number, the period of $\widehat{\mathbf{b}}$ may be a proper divisor $k$ of $n$, and the corresponding address may again be either infinite or finite with length $k$. Thus we have a disjoint union

$$
\Sigma_{n}^{d}=\dot{\Sigma}_{n}^{d} \cup \Sigma_{n, \infty}^{d} \cup \underset{k \mid n, k<n}{\bigcup} \Sigma_{k}^{d} .
$$


If the address $B$ is realized by an existing hyperbolic sector $\mathcal{B}$, then $\widehat{\mathbf{b}}$ is its root sequence as defined above (4.3), and $\varkappa_{d}^{-1}(\widehat{\mathbf{b}})$ is its root address (4.7).

5.8. Primitive and satellite addresses. The internal address and kneading sequence of a satellite hyperbolic component $\mathcal{B}$ with period $n=q k$ are of the form

$$
\begin{aligned}
B & =1\left(s_{1}\right) \longmapsto \ldots \longmapsto k(s) \longmapsto q k, \\
K_{d}(\mathcal{B}) & =\varkappa_{d}(B)=\overline{\left(c_{1} \ldots c_{k-1} c_{k}\right)^{q-1} c_{1} \ldots c_{k-1} *},
\end{aligned}
$$

where $\widehat{K}_{d}(\mathcal{B})=\overline{c_{1} \ldots c_{k}}$ is the kneading sequence of the $k$-periodic parent component $\mathcal{C}$ where $\mathcal{B}$ bifurcates from. On the other hand, the internal address and kneading sequence of a primitive hyperbolic component are not of this form: instead, its root sequence has the same exact period $\langle\mathcal{B}\rangle$ and translates in LSA into an infinite sequence of sectors approaching it from below.

We now extend this concept for formal addresses and thus for nonexistent components. Thus, an address $B \in \Lambda_{n}^{d}$ (and the component $\mathcal{B}$ ) is

- primitive if its root sequence $\widehat{\mathbf{b}}$ is in $\Sigma_{n, \infty}^{d}$, and

- satellite if $\widehat{\mathbf{b}} \in \dot{\Sigma}_{k}^{d}$ for some $k \mid n$.

5.9. Shadow satellites. For the root sequence of a formal address $B$, there is a third possibility: $\widehat{\mathbf{b}} \in \Sigma_{k, \infty}^{d}$ for some $k \mid n$. Then $\mathbf{b} \in \dot{\Sigma}_{q k}^{d}$ consists of a primitive root address $\overline{c_{1} \ldots c_{k}^{\prime}} \in \Sigma_{k, \infty}^{d}$ chopped just before the index $n=q k$ and $c_{q k}$ changed. Such a component $\mathcal{B}$ is a shadow satellite of the $k$-periodic sector $\mathcal{C}$ with kneading sequence $\overline{c_{1} \ldots c_{k}} \in \dot{\Sigma}_{k}^{d}(\mathcal{C}$ may or may not exist).

Of the sequences in $\Sigma_{6}^{d}$ (compare to Example 5.5),

$$
\varkappa_{d}^{-1}(\overline{\mathbf{1 0 1 1 0 j}})=1(1) \longmapsto 2(d-1) \longmapsto 4(1) \longmapsto 5(d-1) \longmapsto 6(j-1) \quad(j \neq 1)
$$

is an example of a shadow satellite's sector. The root sequence of its parent is $\varkappa_{d}^{-1}(\overline{\mathbf{1 0 1}})=1(1) \longmapsto 2(d-1) \longmapsto 4(1) \longmapsto 5(d-1) \longmapsto 7(1) \longmapsto 8(d-1) \longmapsto$ $10(1) \longmapsto 11(d-1) \ldots$

5.10. Lemma. Shadow satellites are nonexistent.

Proof. Suppose we had a component $\mathcal{B}$ with period a proper multiple $q k$ of $k$ such that $\hat{\varkappa}_{d}(B)=\overline{b_{1} \ldots b_{k}} \in \Sigma_{k}^{d} \backslash \dot{\Sigma}_{k}^{d}$. All hyperbolic components are either primitive or satellite. If $\mathcal{B}$ were a primitive component, the exact period of $\hat{\varkappa}_{d}(B)$ would be $q k$; if it were a satellite of a $k$-periodic sector, then $\hat{\varkappa}_{d}(B)$ would be the kneading sequence of this sector and thus would have to belong to $\dot{\Sigma}_{k}^{d}$.

5.11. Lemma. For any natural numbers $k$ and $q \geq 2$, every primitive hyperbolic component (existent or not) with period $k$ has a shadow satellite 
of period qk. Conversely, the parent component of any shadow satellite is primitive.

Proof. Let the parent component have kneading sequence $\mathbf{c}:=\overline{c_{1} \ldots c_{k-1}}$ * and address $C=1\left(\underline{\left.s_{1}\right) \longmapsto \ldots \longmapsto k}\right.$. Then all sequences $\overline{c_{1} \ldots c_{k}}$ with $c_{k} \in$ $\mathbb{Z}_{d}$ except one, say $\overline{c_{1} \ldots c_{k}^{\prime}}$, correspond to the $d-1$ sectors of the same component (and thus have exact period $k$ ). If $C$ has a shadow satellite, its kneading sequence is $\left(c_{1} \ldots c_{k}^{\prime}\right)^{q-1} c_{1} c_{2} \ldots *$ for some $q \in \mathbb{N}$.

If $C$ is primitive, then the root sequence $\overline{c_{1} \ldots c_{k}^{\prime}}$ also has exact period $k$. Now $\overline{c_{1} \ldots c_{k}^{\prime}} \in \Sigma_{k}^{d} \backslash \dot{\Sigma}_{k}^{d}$, so $\overline{\left(c_{1} \ldots c_{k}^{\prime}\right)^{q-1} c_{1} \ldots c_{k}} \in \dot{\Sigma}_{q k}^{d}$ corresponds to a shadow satellite address.

If $C$ is a satellite address, then the exact period of the root sequence, $\overline{c_{1} \ldots c_{k}^{\prime}}=\overline{\left(c_{1} \ldots c_{n}\right)^{r}}=\overline{c_{1} \ldots c_{n}}$, is a proper divisor $n=k / r$ of $k$. This formal kneading sequence belongs to the $n$-periodic parent sector $\mathcal{N}$ of $\mathcal{C}$, and the sequence $\overline{\left(c_{1} \ldots c_{k}^{\prime}\right)^{q-1} c_{1} \ldots c_{k}}=\overline{\left(c_{1} \ldots c_{n}\right)^{q r-1} c_{1} \ldots c_{n}^{\prime}}$ belongs to a $q r n$-periodic satellite of $\mathcal{N}$, rather than to a shadow satellite. Therefore satellite components do not have shadow satellites.

5.12. REMARK. We have thus found one infinite class of nonexistent hyperbolic components, namely the shadow satellites of primitive components. It follows that every primitive component has one set of satellites bifurcating from each of its $d-1$ sectors, and one more set of shadow satellites.

The following somewhat technical result describes a regularity property of primitive root addresses: the sequence of differences between consecutive numbers in the rough address, $\left(n_{i+1}-n_{i}\right)_{i \in \mathbb{N}}$, is (pre)periodic.

5.13. Lemma. If $\mathbf{b}=\overline{b_{1} \ldots b_{n}} \in \Sigma_{n, \infty}^{d}(n \geq 3)$ and the corresponding root address is $\varkappa^{-1}(\mathbf{b})=1\left(s_{1}\right) \longmapsto n_{2}\left(s_{2}\right) \longmapsto \ldots$, then a number $N>2 n$ occurs in the address if and only if $N=n_{i}+$ tn for some $i, t \in \mathbb{N}$ such that $n<n_{i}<2 n$. Moreover, the sector number $s_{N}$ equals $s_{i}$.

Proof. We make two preliminary observations. First, none of the $n_{i}$ can be an integer multiple of $n$; otherwise the address would be finite. Second, there must be some $i$ such that $1<n_{i}<n$; otherwise $\mathbf{b}=\overline{b_{1}} \in \dot{\Sigma}_{1}^{d}$ contrary to the assumption. If we denote the indices so that $1<n_{j}<n<n_{j+1} \leq$ $n_{h}<2 n<n_{h+1}$, the statement will follow from these two results:

(i) $n_{h}=n_{j}+n$.

(ii) If $n_{m}=n_{i}+n$ for some $i, m$, then $n_{m+1}\left(s_{m+1}\right)=\left(n_{i+1}+n\right)\left(s_{i+1}\right)$.

To prove (i), note that by definition (5.3) the first $n_{j+1}-1 \geq n$ figures in $\mathbf{b}$ and $\overline{b_{1} \ldots b_{n_{j}}}$ agree; in particular,

$$
b_{1} \ldots b_{n-n_{j}}=b_{n_{j}+1} \ldots b_{n}=b_{n_{j}+n+1} \ldots b_{2 n}
$$

(the last equality follows from the $n$-periodicity of $\mathbf{b}$ ). Now the first $2 n$ figures 
in $\mathbf{b}$ and $\overline{b_{1} \ldots b_{n_{j}+n}}$ agree, so the maximal period number less than $2 n$ in the root address, $n_{h}$, is at most $n_{j}+n$. On the other hand, $n-n_{j}$ is the maximal number of same figures in the beginning and end of the word $b_{1} \ldots b_{n}$, and thus also the word $b_{1} \ldots b_{n} \ldots b_{2 n}$, so $n_{h}=2 n-\left(n-n_{j}\right)=n_{j}+n$. In the following expression, the braced subwords are equal:

$$
\mathbf{b}=\underbrace{b_{1} b_{2} \ldots} b_{n_{(j+1)}-n_{j}} \ldots b_{n_{j}} \underbrace{\ldots b_{n} \ldots} b_{n_{(j+1)}} \ldots b_{n_{h}} \underbrace{}_{n_{i+1} \ldots b_{2 n} \ldots} b_{n_{(h+1)}} \ldots
$$

To prove (ii), note that if $n_{i+1}=n_{i}+1$, then $b_{1}+s_{i+1}=b_{n_{(i+1)}}=$ $b_{n_{(i+1)}+n}$. The first index at which $\mathbf{b}$ and $\overline{b_{1} \ldots b_{n_{i}+n}}$ differ is $n_{m+1}$; the $\left(n_{i}+n+1\right)$ th figure of the latter is $b_{1}=b_{n_{(i+1)}+n}-s_{i+1}$, so $n_{m+1}=n_{i+1}+n$. Otherwise $n_{i+1}-n_{i}>1$, and

$$
\begin{aligned}
b_{n_{i}+1} \ldots b_{n_{(i+1)}-1} & =b_{1} \ldots b_{n_{(i+1)}-n_{i}-1}, \\
b_{n_{(i+1)}}-s_{i+1} & =\left.\overline{b_{1} \ldots b_{n_{i}}}\right|_{n_{(i+1)}}=b_{n_{(i+1)}-n_{i}} .
\end{aligned}
$$

Adding $n$ to the indices preserves the equality, so the first $n_{i+1}-n_{i}-1$ figures of $\mathbf{b}$ equal $b_{n_{m}+1} \ldots b_{n_{(i+1)}+n-1}$, and $b_{n_{(i+1)}-n_{i}}=b_{n_{(i+1)}+n}-s_{i+1}$. Hence the first difference between $\mathbf{b}$ and $\overline{b_{1} \ldots b_{n_{m}}}$ occurs at index $n_{m+1}=$ $n_{m}+n_{i+1}-n_{i}=n_{i+1}+n$; the difference $b_{n_{(m+1)}}-\left.\overline{b_{1} \ldots b_{n_{m}}}\right|_{n_{(m+1)}}=s_{i+1}$ is the sector number $s_{m+1}$.

5.14. REMARK. In other words, starting from some index less than $2 n$, the same period numbers modulo $n$ and the same sector numbers modulo $d$ keep recurring infinitely. Example 5.5 shows that the sequence $\left\{n_{1}, \ldots, n_{j}\right\}$ is not necessarily equal to $\left\{n_{j+1}, \ldots, n_{h}\right\}$ modulo $n$, so the increment sequence $\left(n_{i+1}-n_{i}\right)_{i \in \mathbb{N}}$ may be strictly preperiodic. For the twelve infinite root addresses in 5.5, the increment sequences are

$$
\begin{array}{llll}
4, \overline{5,1} ; & 2, \overline{2,3,1} ; & \overline{1,3,2} ; & \overline{1,1,2,2} ; \\
3, \overline{4,2} ; & \overline{2,1,3} ; & 1, \overline{2,4} ; & \overline{1,1,1,3} ; \\
3, \overline{1,4,1} ; & 2, \overline{1,1,3,1} ; & \overline{1,2} ; & \frac{1,1,1,1,2}{1,}
\end{array}
$$

Note that the sum of the increments in the periodic part (overlined above) is always the exact period of the kneading sequence; it is six in this example, except for the shadow satellite.

5.15. Period and subaddress. We will extend some more definitions regarding existent parameters and their internal addresses to formal addresses - and thus nonexistent parameter classes - like we did above with primitiveness and satelliteness.

The period of a nonexistent component is the length of its address: $\langle\mathcal{B}\rangle:=|B|$.

Any formal address $B=1\left(s_{1}\right) \longmapsto n_{2}\left(s_{2}\right) \longmapsto \ldots \in \Lambda^{d}$ determines a (finite or infinite) sequence of finite subaddresses $B_{j}=1\left(s_{1}\right) \longmapsto n_{2}\left(s_{2}\right) \longmapsto \ldots \longmapsto$ $n_{j}\left(s_{j}\right) \in \Lambda_{n_{j}}^{d}$ of $B$. 
5.16. Partial ordering of addresses. We define the "above"-relation recursively as follows:

- If $B$ is a subaddress of $A$, then $B \prec A$.

- If $A, C$ are formal addresses and $C \prec A$, then comparing the kneading sequences $\widehat{\varkappa}_{d}(A)$ and $\varkappa_{d}(C)$, LSA yields a unique formal kneading sequence and the corresponding finite address $B$; now $C \prec B \prec A$.

- If $B$ is an infinite address and $B_{j} \prec A$ for all the subaddresses $B_{j}$ of $B$, then $B \prec A$.

These conditions are evidently satisfied by the internal addresses of existent parameter classes that are ordered by the natural partial relation (3.1), so this new relation is its extension. Combinatorial arc $[C, A]$ and visibility are now defined similarly to 3.2 .

Any formal address can be joined to $1\left(s_{1}\right)$ by a unique combinatorial arc. Note that uniqueness follows directly from the definition (second point of 5.16): given two symbolic sequences, the place of their first difference determines a unique address between them (cf. 5.2). In the Mandelbrot set we needed Lavaurs's Lemma (3.3) to guarantee that the corresponding hyperbolic sector is also unique; nonexistent sectors, on the other hand, are just formal addresses, for which there is no ambiguity to begin with. Hence, $\Lambda^{d}$ is a partially ordered, simply connected topological space.

5.17. Remark. If $A$ and $C$ are any formal addresses, one can use LSA to study whether they are comparable in $\prec$. If their root kneading sequences differ, then necessarily after a finite number of iterations one either finds that $A \in \overline{\left[1\left(s_{1}\right), C\right]}$ or else there is a finite address $\left.B \in\right] 1\left(s_{1}\right), C[$ such that $B \notin] 1\left(s_{1}\right), A[$.

For example, consider the two addresses $A=1 \longmapsto 2 \longmapsto 4 \longmapsto 5 \in \Lambda_{5}^{2}$ and $B=1 \longmapsto 2 \longmapsto 4 \longmapsto 6 \longmapsto 9 \in \Lambda_{9}^{2}$. Clearly $B$ cannot be above $A$, because the minimal period of components between $B$ and $C_{4}:=1 \longmapsto 2 \longmapsto 4$ is six and not five. Thus either $B \prec A$, or they diverge at $C_{4}$ or above it. Now the root sequence of $A$ and its root address are, respectively, $\overline{\mathbf{1 0 1 1 1}}$ and $1 \longmapsto 2 \longmapsto 4 \longmapsto 6 \longmapsto 7 \longmapsto 9 \ldots$

Because $B$ does not contain the number seven, $B$ or the branching point must be found between $C_{6}$ and $C_{7}$. Thus we compare

$$
\widehat{\varkappa}_{2}\left(C_{7}\right)=10111111011111 \ldots \text { with } \quad \varkappa_{2}\left(C_{6}\right)=101111101111 \ldots
$$

to find their first difference at the 8 th digit. Because $B$ does not contain the number eight either, we then compare

$$
\widehat{\varkappa}_{2}\left(C_{8}\right)=1011111010111110 \ldots \text { with } \varkappa_{2}\left(C_{6}\right)=101111101111 \ldots
$$

They first differ at the 10th digit; $C_{4} \prec C_{6} \prec C_{10} \prec C_{8} \prec C_{7} \prec A$ and 
$C_{4} \prec C_{6} \prec B$. If $B$ were below $A$, the number nine should have occurred by now. Since it has not, $B$ must branch off the arc $] 1, A[$ at some point.

The visible-tree algorithm we present in $\S 6$ will enable us find such combinatorial relations much more easily, making this kind of complicated calculations largely unnecessary.

5.18. Remark. The root sequences of two different components can only be identical if $\mathcal{A}$ is a shadow satellite of $\mathcal{C}$ : if we have an $n$-periodic root sequence $a_{1} a_{2} \ldots$, we get the kneading sequence of the component by replacing either $a_{n}$ or $a_{2 n}, a_{3 n}$, etc. by $*$. The word $a_{1} \ldots a_{n-1} *$ repeated periodically yields a primitive component, whereas $a_{1} \ldots a_{n} a_{1} \ldots a_{q n-1} *$ gives its shadow satellite of period $q n$. Now neither component is above the other, but $\mathcal{A}$ is visible from (and hence above) any subaddress of $\mathcal{C}$ 's root whose length is more than $\langle\mathcal{A}\rangle$.

5.19. Vanishing point. Every formal address starts with $1\left(s_{1}\right)$ for some $s_{1}$, which is realized by a sector of the main epicycloid. If an address $A$ is realized, then so must be anything below it, that is, the whole closure of the combinatorial arc $\left[1\left(s_{1}\right), A\right]$.

On the other hand, if an address $E \in \Lambda^{d}$ is not realized, then neither is any address above it (by uniqueness of combinatorial arcs). Therefore there must be a unique point in the closure of $\left[1\left(s_{1}\right), E\right]$ which divides the combinatorial arc into realizable and nonrealizable parts. This parameter $v \in$ $\mathcal{M}^{d} / \simeq$, at which the arc jumps from the complex plane into nonexistence, is called the vanishing point of the nonexistent component $\mathcal{E}$.

5.20. Shadow component. We have seen that a component $\mathcal{E}$ is nonexistent if it is a shadow satellite or sits above one. We call such components shadow components and find more of them in the next section. The definitions above imply the following characterization:

5.21. Lemma. Let $\mathcal{E}$ be a nonexistent component. Then $\mathcal{E}$ is a shadow component if and only if its vanishing point is the root of some existent component $\mathcal{B}$.

5.22. REMARK. Being a shadow component is thus a sufficient condition for a component to be nonexistent for all degrees $d \geq 2$. In the quadratic case $d=2$, this is also a necesssary condition by [B-S]. For higher degrees this is open.

Given a kneading sequence a, Henk Bruin and Dierk Schleicher define [B-S, 2.2] a mapping $\varrho: \mathbb{N} \rightarrow \mathbb{N} \cup\{\infty\}$ by $\varrho(n):=\inf \left\{k>n: a_{k} \neq a_{k-n}\right\}$, and $\operatorname{orb}_{\varrho}(n):=n \longmapsto \varrho(n) \longmapsto \varrho \circ \varrho(n) \longmapsto \ldots$; then $\operatorname{orb}_{\varrho}(1)$ is the corresponding formal address as defined here in 5.2. A kneading sequence $\mathbf{e}$ and its address $E \in \Lambda_{n}^{2}$ are said to fail the admissibility condition for period $n$ if [B-S, 2.2] 
(1) $E$ does not contain $n$,

(2) if $k<n$ divides $n$, then $\varrho(k) \leq n$,

(3) if $r \leq n$ is congruent to $\varrho(n)$ modulo $n$, then $\operatorname{orb}_{\varrho}(k)$ contains $n$.

This is shown to be equivalent to the nonexistence of the component $E$. These conditions also imply that $E$ is a shadow component [B-S, 6.5].

\section{GROWING FORMAL TREES}

We know from the theory of internal addresses [L-S-1] that a kneading sequence contains information of which way one had to walk in order to reach the sector with that kneading sequence. It turns out that the kneading sequence also contains information about what lies ahead. There is a Visible Tree Algorithm (VTA, 6.4) by which one can find the combinatorial structure of the tree of all formal addresses that are "potentially" visible from a given base sector, existent or not.

Unfortunately, some components given by VTA do not actually exist in $\mathcal{M}^{d}$ even if the base does. However, we now grow the whole trees with our algorithm and study their properties for their own right, then "prune" nonexistent twigs in $\S 8$.

6.1. Visibility over a sector. Let $\mathcal{A}$ and $\mathcal{B}$ be hyperbolic sectors with periods $m$ and $n$ respectively, such that $\mathcal{B} \prec \mathcal{A}, n>m$, and no sector on ] $\mathcal{B}, \mathcal{A}[$ has period less than $m$. Then we say that $\mathcal{A}$ is visible over $\mathcal{B}$. If no sector on $] \mathcal{B}, \mathcal{A}[$ has period less than $n$, then $\mathcal{A}$ is immediately visible over $\mathcal{B}$.

These definitions are motivated by the fact that such a sector $\mathcal{A}$ is visible from both $\mathcal{B}$ and any sector $\mathcal{C}$ below $\mathcal{B}$ from where $\mathcal{B}$ is, in turn, visible (by definitions 3.2 and 5.16; note that we need not assume the sectors in question to exist). Moreover, no sector on the combinatorial arc $] \mathcal{B}, \mathcal{A}[$ is visible from $\mathcal{C}$ if $\mathcal{A}$ is immediately visible from $\mathcal{B}$.

In any visible tree, each hyperbolic sector is obviously immediately visible over the preceding sector. Our tree-growing algorithm will be based on the following lemma, which gives a necessary condition for a hyperbolic sector to sit above another, immediately visible over it: the periodic word of the lower one begins and ends with the same subword.

6.2. LEMmA. If $\mathcal{A}$ is a hyperbolic sector immediately visible over another sector $\mathcal{B}$ with $\langle\mathcal{A}\rangle=m<n=\langle\mathcal{B}\rangle$, then the periodic word $\left.K(\mathcal{B})\right|_{n}=$ $b_{1} \ldots b_{n}$ has the same subword of length $l:=n-m$ at both ends, i.e., $b_{1} \ldots b_{l}=b_{m+1} \ldots b_{n}$. Moreover, $m \nmid n$.

Proof. By definition of immediate visibility, $\mathcal{A}$ and $\mathcal{B}$ are not separated by parameter rays with periods less than or equal to $n$, so (by 2.6) the first $n$ digits of the sequences $\widehat{K}(\mathcal{A})$ and $K(\mathcal{B})$ must agree. Hence the kneading 
sequences of $\mathcal{A}$ and $\mathcal{B}$ differ exactly at indices that are multiples of $m$ :

$$
b_{1} b_{2} \ldots b_{m} \ldots b_{n}=a_{1} a_{2} \ldots a_{m}^{\prime} a_{m+1} \ldots a_{n} .
$$

If $n=j m$ for some $j \in \mathbb{N}$, this would imply $K(\mathcal{B})=\overline{\left(a_{1} a_{2} \ldots a_{m}^{\prime}\right)^{j}}=$ $\overline{a_{1} a_{2} \ldots a_{m}^{\prime}} \in \Sigma_{m, \infty}^{d}$, which is impossible. Because $\widehat{K}(\mathcal{A})$ is $m$-periodic, (6.2a) yields

$$
\begin{aligned}
b_{m+1} \ldots b_{2 m} \ldots b_{n} & =a_{m+1} \ldots a_{2 m}^{\prime} \ldots a_{n} \\
& =a_{1} \ldots a_{m}^{\prime} \ldots a_{n-m}=b_{1} \ldots b_{l} .
\end{aligned}
$$

It follows directly that an address not satisfying this subword condition can have no components with less periods above it, so it must be realized by a narrow hyperbolic sector (if at all). (The converse statement, that all narrow sectors have narrow addresses, will be proved in 8.4.) We can now define:

6.3. Narrow address. A formal address $B \in \Lambda_{n}^{d}$ is narrow if its periodic word $b_{1} \ldots b_{n}$ does not begin and end with the same subword of any length.

Lemma 6.2 can be used iteratively to find the addresses of all hyperbolic components that may be visible from a given sector $\mathcal{C}$, starting with the satellite components, proceeding to primitive components immediately visible over the satellites, and so on.

Finding all formal addresses that satisfy the necessary subword-condition, we can now construct the formal tree based at a given finite formal address, from the stem to branch-tips. Because we are only dealing with the internal addresses and not with any analytical information about the parameter plane or even external angles, this works for nonexistent base sectors as well as existent ones.

6.4. Visible Tree Algorithm (VTA). Let $\mathcal{C}$ at the formal $d$-address $C=1\left(s_{1}\right) \longmapsto \ldots \longmapsto k\left(s_{k}\right)$ with $\varkappa_{d}(C)=\overline{c_{1} \ldots c_{k}}$ be the base sector. Fix denominator $q$ of the internal angle. For each sector number $s$, find the tree's stem (the $q$-satellite)

$$
C_{q}^{s}=1\left(s_{1}\right) \longmapsto \ldots \longmapsto k\left(s_{k}\right) \longmapsto q k(s)
$$

with $\varkappa_{d}\left(C_{q}^{s}\right)=\overline{\left(c_{1} \ldots c_{k}\right)^{q-1} c_{1} \ldots\left(c_{k}+s\right)}$ by changing the $q k$ th digit in the kneading sequence from $c_{q k}=c_{k}$ into $c_{k}+s$.

Find all numbers $l_{0}$ such that $c_{1} \ldots c_{l_{0}}=c_{q k-l_{0}+1} \ldots\left(c_{q k}+s\right)$; there are obviously a finite number of such $l_{0}$ 's. For each $l_{0}$ there is a primitive address $A$ immediately above the stem with period $m:=q k-l_{0}$ and root kneading sequence $\overline{c_{1} \ldots c_{m}}=: \overline{a_{1} \ldots a_{m}^{\prime}}$. Replacing $c_{m}=a_{m}^{\prime}$ with the other numbers $a_{m} \in \mathbb{Z}_{d}$, one gets the kneading sequences of respective sectors of $\mathcal{A}$. 
Then, for all addresses found in this way, find every number $l_{1}$ such that $a_{1} \ldots a_{l_{1}}=a_{m-l_{1}+1} \ldots a_{m}$, chop off the last $l_{1}$ digits in the periodic word and change the $\left(m-l_{1}\right)$ th one to obtain the kneading sequences $\overline{a_{1} \ldots\left(a_{m-l_{1}}+s\right)}$ $\left(s \in \mathbb{Z}_{d} \backslash\{0\}\right)$, and so on, until no more such l's can be found.

We call the resulting set the formal tree $\widetilde{\mathcal{T}}_{. / q}$ of $C$ (or $\mathcal{C}$ ). It is not necessarily combinatorially equivalent to the "real" tree $\mathcal{T}_{p / q}$, but it contains a subtree which is. The following examples together with Figure $G$ illustrate the action of the algorithm.

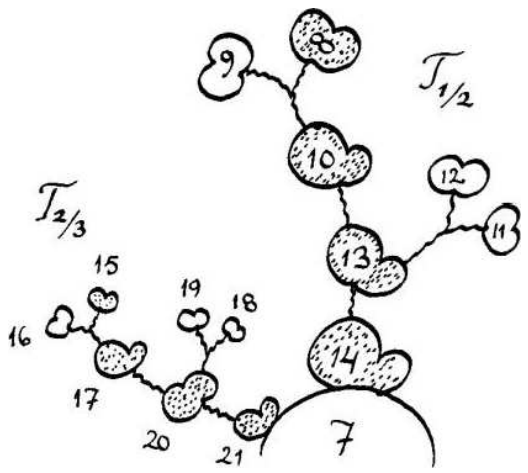

Fig. G

6.5. Example. Consider the address $C=1(1) \longmapsto 3(1) \longmapsto 6(1) \longmapsto$ $7(1) \in \Lambda_{7}^{3}$ with $\varkappa_{3}(C)=\overline{\mathbf{1 1 2 1 1 0 2}}$. The periodic word 11211021121100 of the first sector of the satellite $C_{2}^{1} \in \Lambda_{14}^{3}$ has no common subwords at both ends, so the sector is narrow. The second sector has "1" at both ends, so there is a 13-periodic component above it.

Its first sector has periodic word 1121102 112111, which starts and ends with both "11" and "1", giving rise to adjacent addresses of lengths $13-2=11$ and $13-1=12$, respectively. These are both narrow.

The second sector of the address in $\Lambda_{13}^{3}$ has periodic word 1121102 112112 with "112" at both ends, so it precedes a ten-periodic component whose first sector $(\mathbf{1 1 2 1 1 0 2} \mathbf{1 1 0})$ is narrow and the second precedes a pair of adjacent narrow components with periods eight and nine. Using the joker symbol $*$ as in 5.4 , we write briefly:

\begin{tabular}{|c|c|}
\hline 21 & 1(1) $\longrightarrow 2(1) \longrightarrow 6(1) \longrightarrow 7$ \\
\hline 211021121101 & $1(1) \longmapsto 3(1) \longmapsto 6(1) \succ 7(1) \longmapsto 14(2)$ \\
\hline 121102112111 & $1(1) \longmapsto 3(1) \longmapsto 6(1) \succ 7(1) \longmapsto 13(1)$ \\
\hline $121102112 *$ & $1(1) \longmapsto 3(1) \longmapsto 6(1) \longmapsto 7(1) \longmapsto 11$ \\
\hline $1211021121 *$ & $1(1) \longmapsto 3(1) \longmapsto 6(1) \longmapsto 7(1) \longmapsto 12$ \\
\hline 121102 & $1(1) \longmapsto 3(1) \longmapsto 6(1) \longmapsto 7(1) \longmapsto 13(2)$ \\
\hline
\end{tabular}




\begin{tabular}{|c|c|}
\hline 1121102110 & $1(1) \longmapsto 3(1) \longmapsto 6(1) \longmapsto 7(1) \longmapsto 10(1)$ \\
\hline 1121102111 & $1(1) \longmapsto 3(1) \longmapsto 6(1) \longmapsto 7(1) \longmapsto 10(2)$ \\
\hline $1121102 *$ & $1(1) \longmapsto 3(1) \longmapsto 6(1) \longmapsto 7(1) \longmapsto 8$ \\
\hline $11211021 *$ & $1(1) \longmapsto 3(1) \longmapsto 6(1) \longmapsto 7(1) \succ 9$ \\
\hline
\end{tabular}

6.6. Lemma. The formal tree $\widetilde{\mathcal{T}}_{\text {./2 }}$ of the address

$$
C=1\left(s_{1}\right) \longmapsto n_{2}\left(s_{2}\right) \longmapsto \ldots \longmapsto n_{k-1}\left(s_{k-1}\right) \longmapsto n_{k}\left(s_{k}\right)
$$

contains a chain of formal addresses $A_{j}$ such that $A_{j} \in \Lambda_{n_{k}+n_{j}}^{d}$ for all $j \in$ $\{1, \ldots, k\}$ and $A_{1} \succ A_{2} \succ \ldots \succ A_{k-1} \succ A_{k}=C_{2}$. Each $A_{j}$ is immediately visible over $A_{j+1}$, and the sector number of $A_{j}$ equals $d-s_{j}$.

(This lemma was inspired by an observation by Dierk Schleicher, p.c.)

Proof. In the periodic word $c_{1} \ldots c_{n_{2}} \ldots c_{n_{(k-1)}} \ldots c_{n_{k}}$ of $C$, by definition,

$$
c_{n_{k}}-s_{k}=\left(\overline{c_{1} \ldots c_{n_{(k-1)}}}\right)_{n_{k}}=\left(\overline{c_{1} \ldots c_{n_{(k-1)}}}\right)_{n_{k}-n_{(k-1)}}=c_{n_{k}-n_{k-1}}
$$

( $c_{i}$ modulo $d$, as always). On the other hand, the periodic word of the satellite $A_{k}$ 's sector number $r_{k}$ ends with $c_{n_{k}}+r_{k}$. Thus one sector, namely number $d-s_{k}=r_{k}$, has the subword $c_{1} \ldots c_{n_{k}-n_{(k-1)}}$ at both ends of its periodic word. This means that there is a formal address $A_{k-1}$ with length $2 n_{k}-$ $\left(n_{k}-n_{k-1}\right)=n_{k}+n_{k-1}$ immediately visible over the stem.

Continuing backwards inductively, if the tree contains a component address $A_{j}$ of length $n_{k}+n_{j}$, then its sector number $r_{j}$ has periodic word

$$
c_{1} \ldots c_{n_{k}} c_{n_{k}+1} \ldots c_{n_{k}+n_{(j-1)}} c_{n_{k}+n_{(j-1)}+1} \ldots\left(c_{n_{k}+n_{j}}+r_{j}\right) \text {. }
$$

The last digit equals $c_{n_{j}}+r_{j}=c_{n_{j}-n_{(j-1)}}+s_{j}+r_{j}$, so the subword $c_{1} \ldots c_{n_{j}-n_{j-1}}$ begins and ends the periodic word of sector number $r_{j}=d-s_{j}$ of $A_{j}$, giving rise to a component address $A_{j-1}$ of length $n_{k}+n_{j-1}$ immediately above $A_{j}$.

6.7. Secondary trunk. The chain in Lemma 6.6 of $n_{k}$ sectors on the combinatorial arc from sector $C$ to its continuation $C \longmapsto n_{k}+1$ is called the secondary trunk of the tree $\widetilde{\mathcal{T}}_{\cdot / 2}(C)$.

For narrow sectors, the primary (3.4) and secondary trunks are obviously the same set (e.g., Figure G), and it can be called just the tree's trunk. For nonnarrow sectors, the secondary trunk may be a subset of the primary trunk (like the tree $\widetilde{\mathcal{T}}_{. / 2}^{2}$ in Figure $\mathrm{J}$ ) or the two trunks may branch off each other (like $\widetilde{\mathcal{T}}_{\cdot / 2}^{1}$ in Figure $\mathrm{J}$ ).

As we saw, for any $s \in \mathbb{Z}_{d} \backslash\{0\}$, the $(d-s)$ th sector of the 2-satellite of the $s$ th sector of the base component has periodic word $c_{1} c_{2} \ldots c_{n_{k}-1}\left(c_{n_{k}}^{\prime}+\right.$ s) $c_{1} c_{2} \ldots c_{n_{k}-1} c_{n_{k}}^{\prime}$, where $\overline{c_{1} \ldots c_{n_{k}}^{\prime}}=\hat{\varkappa}(C) \in \Sigma_{n_{k}, \infty}^{d}$. Because these $d-1$ words of length $2 n_{k}$ differ only at index $n_{k}$, the VT-algorithm proceeds 
identically for all sectors $s$ as long as the addresses remain longer than the base. We have thus proved

6.8. Corollary. Let a component $\mathcal{C}$ (existent or not) be the base. If a sector $\mathcal{A}$ with period $n>n_{k}=\langle\mathcal{C}\rangle$ appears above a sector $\mathcal{B}$ in the secondary trunk of one base sector $\mathcal{C}^{s}$, then another sector $\mathcal{A}^{\prime}$ of the same period $n$ appears above the corresponding sector $\mathcal{B}^{\prime}$ with period $\langle\mathcal{B}\rangle$ in the secondary trunk of any other base sector $\mathcal{C}^{r}$.

For narrow sectors $\mathcal{C}^{s}, \mathcal{C}^{r}$ of the same base component this means that the subtrees stemming at the 2-satellite's sectors $(d-s),(d-r)$ respectively are combinatorially equivalent.

See how the tree in the previous example (6.5) has a chain of addresses with lengths $7+7,7+6,7+3,7+1$ and sector numbers $3-s_{j}$. These are shaded in Figure G (here $k=4, n_{k}=7, j \in\{1,2,3,4\}$ ). See also Figure J, where the secondary trunks and the addresses of lengths 13,17 (>10) branching off it are similar for the two sectors of the 10-periodic base component, but the addresses with lengths less than ten (five and six on the first sector, nine on the second) branch off the tree trunks at different places.

The next lemma restricts the difference between the periods of two consecutive components in the tree - or, from another point of view, restricts the periods of sectors from where such a pair of consecutive sectors can be visible:

6.9. Lemma. Let $\mathcal{A}, \mathcal{B}, \mathcal{C}$ be hyperbolic sectors with periods $m, n, k$ respectively and $l:=n-m$. If $\mathcal{B}$ is visible from $\mathcal{C}$ and $\mathcal{A}$ is immediately visible over $\mathcal{B}$, then $l<k$.

Proof. The sectors $\mathcal{A}$ and $\mathcal{B}$ are in the same formal tree $\widetilde{\mathcal{T}}_{/ q q}$ of $\mathcal{C}$ for some $q ; m<n \leq q k$. By $6.4, \widehat{K}(\mathcal{B})$ and $K(\mathcal{C})$ agree up to at least the $n$th digit. Hence $a_{n}=b_{n} \neq c_{n}$ and

$$
c_{1} \ldots\left(c_{n}+s\right)=b_{1} \ldots b_{n}
$$

where $s \neq 0$ is the sector number of $\mathcal{B}$.

First, assume that $l>k$, which forces $n=m+l>k$. Combining (6.2a), $(6.2 \mathrm{~b})$, and $(6.9 \mathrm{a})$, we obtain $c_{1} \ldots c_{k} \ldots c_{l}=c_{m+1} \ldots c_{m+k} \ldots\left(c_{n}+s\right)$ and hence

$$
c_{k+1} \ldots c_{l}=c_{m+k+1} \ldots\left(c_{n}+s\right) .
$$

On the other hand, $c_{j}=c_{k+j}$ for all $j$, so

$$
c_{k+1} \ldots c_{l}=c_{1} \ldots c_{l-k}=c_{m+1} \ldots c_{m+l-k} .
$$

But since $m+l=n$, the last digit is $c_{n-k}=c_{n} \neq c_{n}+s$, contradicting (6.9b). 
Finally, assume $l=k$. Then $m=i k+r$ and $n=(i+1) k+r$ for some integers $r$ and $i$ such that $0 \leq i \leq q-2,1 \leq r \leq k-1(r>0$ because $\mathcal{A}$ cannot be a satellite of $\mathcal{C}$ or $\mathcal{B})$. Now $(6.2 \mathrm{a})$ and $(6.9 \mathrm{a})$ imply

$$
\begin{aligned}
b_{1} \ldots b_{i k} \ldots b_{(i+1) k} \ldots b_{n} & =\left(c_{1} \ldots c_{k}\right)^{i} c_{1} \ldots c_{k} c_{1} \ldots\left(c_{r}+s\right) \\
& =a_{1} \ldots a_{i k} \ldots a_{m}^{\prime} \ldots a_{n} \\
& =\left(c_{1} \ldots c_{k}\right)^{i} c_{1} \ldots c_{r} c_{1} \ldots\left(c_{k}+t\right)
\end{aligned}
$$

where $t \neq 0$ is the sector number of $\mathcal{A}$. But by $(6.2 \mathrm{~b})$, the last digit should equal $b_{n}=b_{l}=b_{k}=c_{k}$ (because $k=l<n$ and the kneading sequences of $\mathcal{C}$ and $\mathcal{B}$ agree up to the $n$th digit).

6.10. Shadow trees. The VT-algorithm can equally well be used for finding all formal addresses with lengths at most $q k$ which are above the shadow satellites of the given base address $C$ (realizable or not), instead of the proper satellites.

Using the above notation, let $c_{k}^{\prime} \neq c_{k}$ be such that $\overline{c_{1} \ldots c_{k-1} c_{k}^{\prime}} \in \Sigma_{k, \infty}^{d}$. Fixing an integer $q \geq 2$, find all sectors $\widehat{C}_{q}^{s}$ of the shadow $q$-satellite; their kneading sequences are $\varkappa_{d}\left(\widehat{C}_{q}^{s}\right)=\overline{\left(c_{1} \ldots c_{k}^{\prime}\right)^{q-1} c_{1} \ldots\left(c_{k}^{\prime}+s\right)}\left(s \in \mathbb{Z}_{d} \backslash\{0\}\right)$.

Then find all numbers $l$ such that $c_{1} \ldots c_{l}=c_{q k-l+1} \ldots\left(c_{k}^{\prime}+s\right)$. For each such $l$ there is an address $B \in \Lambda_{q k-l}^{d}$ with $\widehat{\varkappa}_{d}(B)=\overline{\left(c_{1} \ldots c_{k}^{\prime}\right)^{q-1} c_{1} \ldots c_{k-l}}$ immediately above the stem; replace the last digit by other numbers in $\mathbb{Z}_{d}$ to get the kneading sequences of all sectors of $B$. Find common parts in both ends of their periodic words, and so forth.

The combinatorial space thus obtained is called the shadow tree $\widetilde{\mathcal{V}}_{\cdot / q}$ of $C$. Because they are above shadow satellites which are nonexistent, the whole shadow trees are nonexistent.

6.11. EXAmplE. Each shadow $q$-tree of the (realizable) formal address $A:=1(1) \longmapsto 2(1) \longmapsto 4(1) \longmapsto 6$ with periodic word $12101 *$ consists of the shadow $q$-satellite and one primitive address of length $(q-1) \cdot 6+5$ above the second sector of the stem; see Figure H.

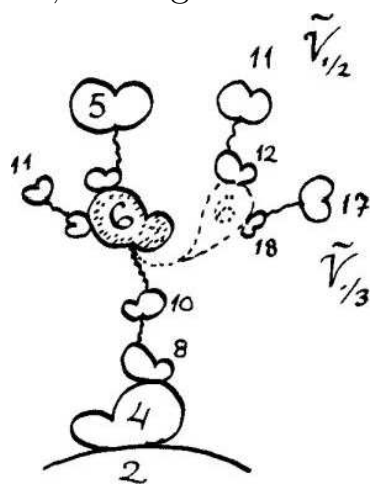

Fig. $\mathrm{H}$ 


\begin{tabular}{|c|c|}
\hline 2101212 & $1(1) \longmapsto 2(1) \longmapsto 4(1) \longmapsto 8(2) \succ 10(1) \longmapsto 12(1)$ \\
\hline 21012121011 & $1(1) \longmapsto 2(1) \longmapsto 4(1) \longmapsto 8(2) \longmapsto 10(1) \longmapsto 12(2)$ \\
\hline $10121210 *$ & $1(1) \longmapsto 2(1) \longmapsto 4(1) \longmapsto 8(2) \longmapsto 10(1) \longmapsto 11$ \\
\hline 2121010 & $1(1) \longmapsto \ldots \longmapsto 10(1) \longmapsto 14(2) \longmapsto 16(1) \longmapsto 18(1)$ \\
\hline 12 & $1(1) \longmapsto \ldots \longmapsto 10(1) \longmapsto 14(2) \longmapsto 16(1) \longmapsto 18(2)$ \\
\hline $210121210 *$ & $1(1) \longmapsto \ldots \longmapsto 10(1) \longmapsto 14(2) \succ 16(1) \longmapsto 17$ \\
\hline
\end{tabular}

6.12. REMARK. In Remark 5.18 we noticed that shadow satellites are visible from the base's root subaddresses with sufficiently large periods. Now we see that the same is true for all primitive components in each shadow tree; they are, by definition, visible over a shadow satellite of the base sector $\mathcal{C}$. Any address $E \in \widetilde{\mathcal{V}}_{q}(\mathcal{C})$ is of the form

$$
1\left(s_{1}\right) \longmapsto n_{2}\left(s_{2}\right) \longmapsto \ldots \longmapsto n_{j}\left(s_{j}\right) \longmapsto h,
$$

where $n_{j}$ is the maximal period of root subaddresses of the base component $\mathcal{C}$ such that $n_{j}<h=|E|$. (By 5.13, $\langle\mathcal{C}\rangle$ does not appear in the root address of $\mathcal{C}$, and hence not in $E$ either.)

Now if $\mathcal{D}$ is a hyperbolic sector realizing some root subaddress of the base $\mathcal{C}$ such that $\langle\mathcal{D}\rangle \geq n_{j}$, then the nonexistent component $\mathcal{E}$ is visible from $\mathcal{D}$ and hence appears in the formal tree given by VTA. In particular, if we move our vantage point to longer and longer finite subaddresses, more and more formal addresses in the shadow trees of the base $\mathcal{C}$ become visible.

For example, see Figures $\mathrm{A}$ and $\mathrm{H}$. The shadow tree of the component at $1(1) \longmapsto 2(1) \longmapsto 4(2) \longmapsto 6$ contains components with periods $9,11,12$. From the subaddress $A=1(1) \longmapsto 2(1) \longmapsto 4(2)$, these three are invisible because the satellite sector $A \longmapsto 8(2)$ is blocking the view. Moving there, the 9-periodic component becomes visible but the ones with periods 11,12 are still behind the primitive sector $A \longmapsto 8(2) \longmapsto 10(2)$. Seen from there, the two finally appear, along with a 17 -periodic component which eventually will turn out to belong to another shadow tree.

6.13. Lemma. If every sector $C^{s}$ of $C$ is narrow, then for each $s$, all but one sectors of the 2-satellite of $C^{s}$ are narrow as well. Moreover, all shadow satellites are narrow, so the shadow trees of $C$ contain no primitive addresses.

(This follows directly from definitions.) The exceptional nonnarrow sector of the 2-satellite is number $d-s$, by Lemma 6.6 .

At this point we can prove a result, "Formal Translation Principle", which generalizes the equivalence statement 3.6 for formal trees and also for shadow trees. (Note that this is not true in general for real visible trees.)

6.14. TheOREM. Let $C$ be a formal address. For any integers $q, q^{\prime}$ greater than 1 , the formal trees $\widetilde{\mathcal{T}}_{. / q}$ and $\widetilde{\mathcal{T}}_{\cdot / q^{\prime}}$ based at $C$ are combinatorially equiv- 
alent. The shadow trees $\widetilde{\mathcal{V}}_{\cdot / q}$ and $\widetilde{\mathcal{V}}_{\cdot / q^{\prime}}$ of $C$ are also combinatorially equivalent.

We divide the proof into a few lemmas about the combinatorial structure of various formal trees and shadow trees.

6.15. LEMMA. If there is a formal address $A$ of length $m<k$ above an address $C \in \Lambda_{k}^{d}$, then $A$ is above the 2-satellite of $C$.

Proof. We may assume that $m$ is maximal in the sense that there are no other addresses with periods less than $k$ between $C$ and $A$. Then $A$ belongs to some formal visible tree $\widetilde{\mathcal{T}}_{\cdot / q}$ of $C$. (Any other addresses with periods less than $k$ above $C$ must then sit above one of such "lowest visible" addresses.) We will show that $q=2$.

The periodic word $c_{1} \ldots c_{k}$ of the base $C$ begins and ends with some subword of length $k-m$. Comparing the root sequence of $A, \overline{c_{1} \ldots c_{m}}$, to $\overline{c_{1} \ldots c_{k}}$, following LSA, one finds the chain of addresses in the visible tree connecting $A$ to $C$. The process will end at the stem whose period is some multiple of $k$. We must show that it ends at $2 k$.

After the first step, the root kneading sequences to be compared with $\overline{c_{1} \ldots c_{k}}$ all have periods greater than $k$. Therefore the process is equivalent to finding the root address of the base $C$. By the same argument and notation as in Lemma 5.13, we see that after finitely many steps the first difference will occur at some index $k+n_{i}$ where $n_{i}<k$ appears in the address $C:=$ $1\left(s_{1}\right) \longmapsto \ldots \longmapsto n_{j}\left(s_{j}\right) \longmapsto k(s)(i \leq j)$. In particular, the algorithm will stop at the period $k+n_{j}$.

The next step will be different from that in 5.13 , because now the $2 k$ th digit is $c_{k} \neq c_{k-n_{j}}$. Therefore the two sequences $\overline{c_{1} \cdots c_{k}}$ and $\overline{c_{1} \cdots c_{k+n_{j}}}$ differ at index $2 k$, so the algorithm ends.
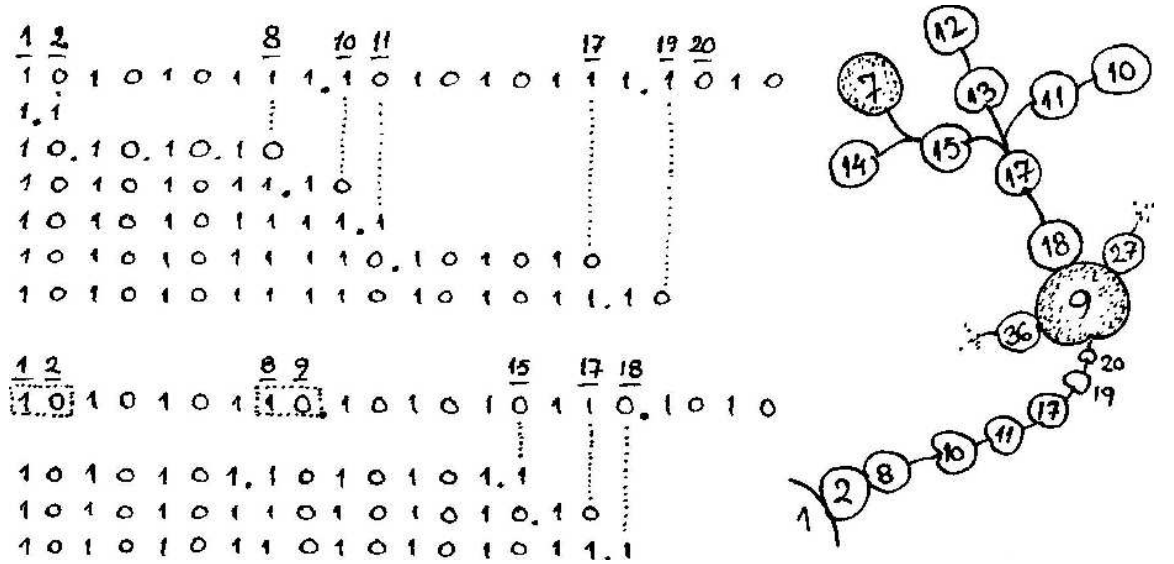

Fig. I 
Note that the argument is also similar to the one in the proof of Lemma 6.6, only the algorithm runs in the opposite direction. Figure I shows the Lau-Schleicher Algorithm from the main cardioid up to a component at $C:=1 \longmapsto 2 \longmapsto 8 \longmapsto 9 \in \Lambda_{9}^{2}$, and from $A:=1 \longmapsto 2 \longmapsto 7$ back down to $C$. See the secondary trunk of the tree with the ten-periodic component at the top, and the primary trunk with $A$ at the top.

The next lemma provides a kind of induction step on the partial order of components, for the proof of Theorem 6.14.

6.16. Lemma. Let $C \in \Lambda_{k}^{d}$ be a base address. The following are equivalent:

- The formal tree $\widetilde{\mathcal{T}}_{\cdot / q}$ of $C$ contains the addresses $B \in \Lambda_{n}^{d}$ and $A \in \Lambda_{m}^{d}$ immediately visible over $B$.

- The formal tree $\widetilde{\mathcal{T}}_{\cdot / q+1}$ of $C$ contains the addresses $B^{\prime} \in \Lambda_{k+n}^{d}$ and $A^{\prime} \in \Lambda_{k+m}^{d}$ immediately visible over $B^{\prime}$, with the sector numbers of $B$ and $B^{\prime}$ equal.

Proof. (Clearly, $m<n \leq q k$.)

(i) Assume that there are addresses $B \in \widetilde{\mathcal{T}}_{/ q q}$ of length $n \leq q k$ and $B^{\prime} \in \widetilde{\mathcal{T}}_{\cdot / q+1}$ of length $k+n$, and that the subtrees constructed up to $B$ and $B^{\prime}$ are equivalent. This is true at least if $B$ and $B^{\prime}$ are the sth sectors of the two trees' stems, respectively.

Because $B$ and $B^{\prime}$ are visible from $C$, the first $n$ figures in the root kneading sequence $\widehat{\varkappa}_{d}(B)$ must agree with those of $\varkappa_{d}(C)=\overline{c_{1} \ldots c_{k}}$, and the first $k+n$ figures in $\widehat{\varkappa}_{d}\left(B^{\prime}\right)$ also agree with those of $\varkappa_{d}(C)$. If we denote the periodic words of $B$ and $B^{\prime}$ respectively by $b_{1} \ldots b_{n}$ and $\beta_{1} \ldots \beta_{k+n}$, this implies that $b_{j}=\beta_{j}=\beta_{k+j} \forall j<n$. Moreover, since the sector numbers are equal, also $b_{n}=\beta_{k+n}\left(\neq \beta_{n}\right)$.

(ii) If there is a formal address $A$ of length $m$ immediately visible over $B$, then by Lemmas 6.2 and $6.9,0<n-m=l<k$ such that $c_{1} \ldots c_{l}$ starts and ends the periodic word of $B$. Together with (i) this implies that $c_{1} \ldots c_{l}$ also starts and ends the periodic word of $B^{\prime}$, so there is a formal address $A^{\prime}$ of length $k+n-l=k+m$ above $B^{\prime}$. This argument is obviously symmetrical with respect to $B$ and $B^{\prime}$, so the converse statement is also true.

6.17. Corollary. For every $q \geq 2$, the formal $q$-tree $\widetilde{\mathcal{T}}_{/ q}$ of the address $C=1\left(s_{1}\right) \longmapsto \ldots \longmapsto k\left(s_{k}\right) \in \Lambda_{k}^{d}$ consists of exactly $k$ component addresses, with lengths in $\{(q-2) k+3, \ldots, q k\}$.

Proof. It follows from 6.16 that the lengths of component addresses in the same tree are all different modulo $k$, so there are at most $k$ of them in each $\widetilde{\mathcal{T}}_{\cdot / q}$. 
If $B \in \widetilde{\mathcal{T}}_{\cdot / 2}$, then $3 \leq|B| \leq 2 k$. For each $j=3, \ldots, k$, the tree $\widetilde{\mathcal{T}}_{\cdot / 2}$ may contain either an address of length $j$ or one of length $k+j$. We must rule out the possibility that it contains neither.

Every formal address with $C$ as its last subaddress is visible from $C$, so let $B:=1\left(s_{1}\right) \longmapsto \ldots \longmapsto k\left(s_{k}\right) \longmapsto k+j$ sit in some formal tree $\widetilde{\mathcal{T}}_{\text {. } / q}$. If $q \geq 3$, Lemma 6.16 implies that $\widetilde{\mathcal{T}}_{\text {. } / q-1}$ contains an address of length $j<k$, so by Lemma $6.15, q=3$. Hence $B$ has exactly two possibilities: either 2 -tree or 3 -tree. In both cases the tree $\widetilde{\mathcal{T}}_{\text {./2 }}$ contains exactly $k$ component addresses, and by 6.16 , so do all other trees.

6.18. REMARK. It is easy to see that the arguments in the proofs of Lemmas 6.16, 6.17 work equally well if "formal tree $\widetilde{\mathcal{T}}_{/ / q}$ ", "satellite $C_{q}^{s}$ ", and " $c_{k}$ " are replaced by "shadow tree $\widetilde{\mathcal{V}}_{\cdot / q}$ ", "shadow satellite $\widehat{C}_{q}$ ", and " $c_{k}^{\prime}$ ", respectively. Hence we have proved Theorem 6.14 .

Now we show a couple of results relating the trees of the various sectors of the same base component. The following lemma reveals a kind of symmetry between the formal trees and the shadow trees of any formal address, so it will also give us a handy tool for sieving nonexistent components from the formal trees.

6.19. Lemma. Let $C=1\left(s_{1}\right) \longmapsto \ldots \longmapsto n_{i}\left(s_{i}\right) \longmapsto \ldots \longmapsto k \in \Lambda_{k}^{d}$ be $a$ formal component address and $s \in\{1, \ldots, d-1\}$ a sector number.

(a) The following conditions are equivalent:

- The formal tree $\tilde{\mathcal{T}}_{\cdot / 2}^{s}$ based at sector $C^{s}$ contains a formal address $B$ with length $|B|=: n<k$ immediately visible over $C$.

- The formal tree $\widetilde{\mathcal{T}}_{\cdot / 2}^{r}$ based at any other sector $C^{r}(r \neq s)$ contains a $B^{\prime}$ with $\left|B^{\prime}\right|=k+n$ such that $n \neq n_{i}$ for all $i ; B^{\prime}$ is immediately visible over the $(s-r)$ th sector of the stem satellite.

- If $C$ is primitive, then the shadow tree $\widetilde{\mathcal{V}}_{\cdot / 2}$ of $C$ contains a $B^{\prime}$ with $\left|B^{\prime}\right|=k+n\left(n \neq n_{i} \forall i\right)$ immediately visible over the sth sector of the stem satellite. If $C$ is a q-satellite of another address $D$, then the formal $2 q$-tree of $D$ contains a $B^{\prime}$ with $\left|B^{\prime}\right|=k+n\left(n \neq n_{i} \forall i\right)$ immediately visible over the sth sector of the stem satellite.

(b) Assuming there are formal addresses $B$ and $B^{\prime}$ as in (a), the tree containing $B$ has an address $A \succ B$ with length $m<n$ if and only if the tree (formal or shadow) containing $B^{\prime}$ also has an $A^{\prime} \succ B^{\prime}$ with $\left|A^{\prime}\right|=k+m$.

Proof. The first condition in (a) implies that the number $n$ cannot appear in the address $C$ : otherwise there would be a subaddress $E \prec C$ with length $n$ such that there were no addresses shorter than $n$ between $E$ and $C$, and not between $C$ and $B$ either, by definition 6.1 . Then the last subad- 
dresses of $E$ and $B$ would be equal, so $E=B$. This contradicts definition 5.16 .

Denoting the root sequence by $\widehat{\varkappa}_{d}(C)=\overline{c_{1} \ldots c_{k-1} c_{k}^{\prime}}$, each sector $s$ has kneading sequence $\varkappa_{d}\left(C^{s}\right)=\overline{c_{1} \ldots c_{k-1}\left(c_{k}^{\prime}+s\right)}$. Then for all $j, r \in \mathbb{Z}_{d} \backslash\{0\}$, the $j$ th sector of the 2 -satellite of sector $C^{r}$ has periodic word

$$
c_{1} \ldots c_{k-n} \ldots c_{k-1}\left(c_{k}^{\prime}+r\right) c_{1} \ldots c_{n} \ldots c_{k-1}\left(c_{k}^{\prime}+r+j\right) \text {. }
$$

For $r=0$, this word belongs to the $j$ th sector of the shadow 2-satellite of $C$ in case $C$ is primitive; otherwise $C$ is a $q$-satellite of $D$, and the word belongs to the $j$ th sector of the $2 q$-satellite of $D$ (cf. 5.9).

All conditions in (a) are satisfied if and only if $j=s-r$ and $c_{1} \ldots c_{k-n}=$ $c_{n+1} \ldots c_{k-1}\left(c_{k}^{\prime}+s\right)$ (by 6.2 and 6.10). (Note that this lemma says nothing about $B^{\prime}$ if it sits in the subtree containing the secondary trunk, because in that case by Lemma 6.6 the satellite sector number would be $d-r$, so $s=d=0$.)

The assumption in (b) means that $\varkappa_{d}(B)=\overline{b_{1} \ldots b_{n}}=\overline{c_{1} \ldots c_{n}^{\prime}} \in \Lambda_{n}$ and $\varkappa_{d}\left(B^{\prime}\right)=\overline{\beta_{1} \ldots \beta_{k+n}}=\overline{c_{1} \ldots c_{k}^{\prime} c_{1} \ldots c_{n}^{\prime}} \in \Lambda_{k+n}$. The first condition in (b) is then satisfied if and only if $b_{1} \ldots b_{n-m}=b_{m+1} \ldots b_{n}$; the second, if and only if $\beta_{1} \ldots \beta_{(k+n)-(k+m)}=\beta_{k+m+1} \ldots \beta_{k+n}$. Since $\beta_{j}=c_{j}=b_{j} \forall j<n$ and the length of these words is $n-m<n$, the two conditions are equivalent.

It follows that the trees stemming from different sectors of a same component differ in addresses with lengths less than that of the base; see Figure $\mathrm{J}$ and recall Lemma 6.8, which said that they all have addresses with the same lengths greater than the base.

6.20. Corollary. Given a base component (address) $C$ and a number $n<|C|$ not appearing in $C$, there is at most one tree based on $C$ containing a formal address of length $n$.

Define $\widetilde{\mathcal{S}}$ as the tree consisting of the base component and components visible over it (an example is right of Figure $\mathrm{J}$ ).

6.21. Corollary. For every $q \geq 2$, the shadow tree $\widetilde{\mathcal{V}}_{\cdot / q}$ of $C$ is combinatorially equivalent to the tree $\widetilde{\mathcal{S}}$ of $C$.

6.22. ExAmple. Let $C=1(1) \longmapsto 2(1) \longmapsto 4(1) \longmapsto 8(2) \longmapsto 10 \in \Lambda_{10}^{3}$ be the base address with $\varkappa_{d}(C)=\overline{\mathbf{1 2 1 0 1 2 1 2 1}} *$. We first grow the formal trees $\widetilde{\mathcal{T}}^{s} / 2$ for the two sectors of $C$; see left of Figure $\mathrm{J}$.

$\begin{array}{ll}12101212101210121211 & 1(1) \longmapsto 2(1) \longmapsto 4(1) \longmapsto 8(2) \longmapsto 10(1) \longmapsto 20(1) \\ 121012121012101212 * & 1(1) \longmapsto 2(1) \longmapsto 4(1) \longmapsto 8(2) \longmapsto 10(1) \longmapsto 19 \\ 12101212101210121212 & 1(1) \longmapsto 2(1) \longmapsto 4(1) \longmapsto 8(2) \longmapsto 10(1) \longmapsto 20(2) \\ 121012121012101210 & 1(1) \longmapsto 2(1) \longmapsto 4(1) \longmapsto 8(2) \longmapsto 10(1) \longmapsto 18(1) \\ 12101212101211 & 1(1) \longmapsto 2(1) \longmapsto 4(1) \longmapsto 8(2) \longmapsto 10(1) \longmapsto 14(1)\end{array}$




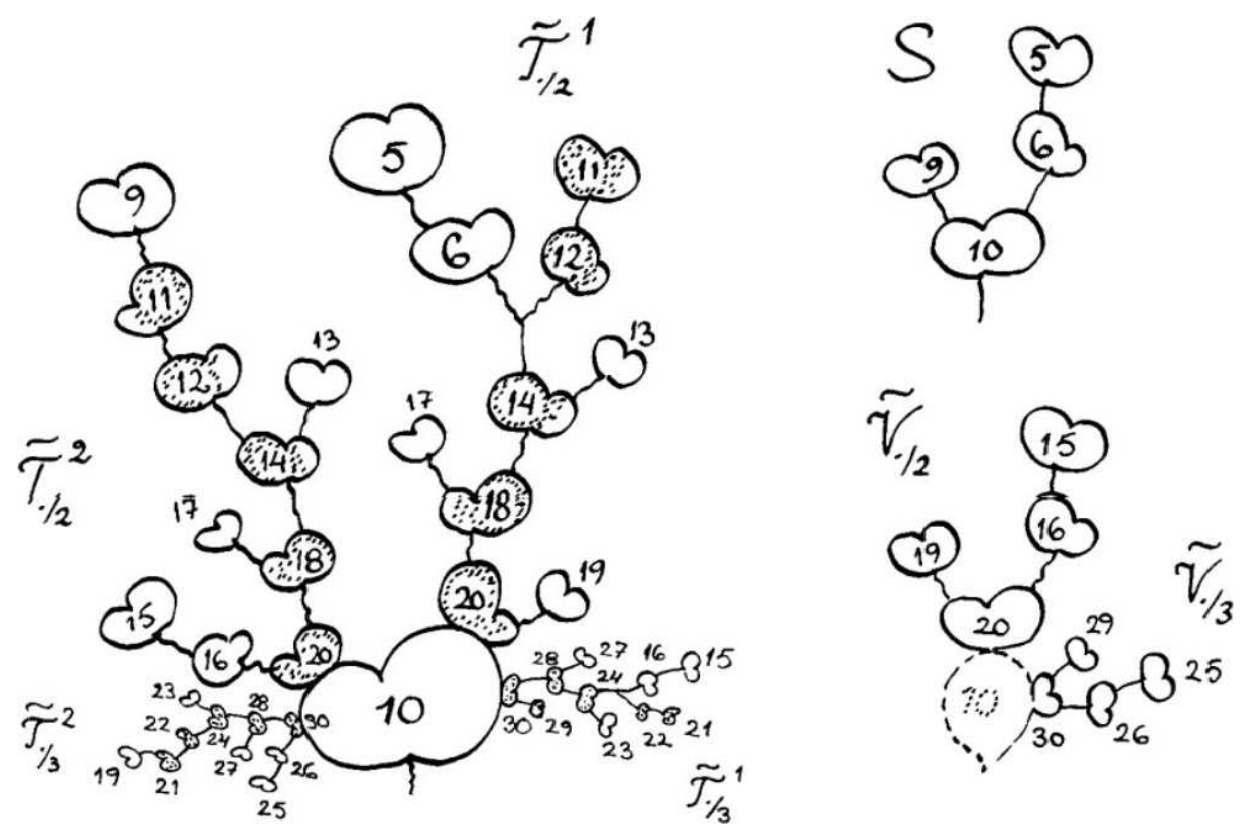

Fig. J

\begin{tabular}{|c|c|}
\hline $121012121012 *$ & $1(1) \longmapsto 2(1) \longmapsto 4(1) \longmapsto 8(2) \succ 10(1) \longmapsto 13$ \\
\hline 12101212101212 & $1(1) \longmapsto 2(1) \longmapsto 4(1) \longmapsto 8(2) \longmapsto 10(1) \succ 14(2)$ \\
\hline 121010 & $1(1) \longmapsto 2(1) \longmapsto 4(1) \longmapsto 6(1)$ \\
\hline 121011 & $1(1) \longmapsto 2(1) \longmapsto 4(1) \longmapsto 6(2)$ \\
\hline $1210 *$ & $1(1) \longmapsto 2(1) \longmapsto 4(1) \succ 5$ \\
\hline 121012121010 & $1(1) \longmapsto 2(1) \longmapsto 4(1) \longmapsto 8(2) \longmapsto 10(1) \longmapsto 12(1)$ \\
\hline 121012121011 & $1(1) \longmapsto 2(1) \longmapsto 4(1) \longmapsto 8(2) \longmapsto 10(1) \longmapsto 12(2)$ \\
\hline $1210121210 *$ & $1(1) \longmapsto 2(1) \longmapsto 4(1) \succ 8(2) \succ 10(1) \succ 11$ \\
\hline 121012121012101211 & $1(1) \longmapsto 2(1) \longmapsto 4(1) \longmapsto 8(2) \longmapsto 10(1) \longmapsto 18(2)$ \\
\hline $1210121210121012 *$ & $1(1) \longmapsto 2(1) \longmapsto 4(1) \longmapsto 8(2) \longmapsto 10(1) \longmapsto 17$ \\
\hline 12101212111210121212 & $1(1) \longmapsto 2(1) \longmapsto 4(1) \longmapsto 8(2) \longmapsto 10(2) \longmapsto 20(1)$ \\
\hline \multicolumn{2}{|l|}{$\cdots$} \\
\hline 12101212112 & $1(1) \longmapsto 2(1) \longmapsto 4(1) \longmapsto 8(2) \longmapsto 10(2) \longmapsto 11(1)$ \\
\hline $12101212 *$ & $1(1) \longmapsto 2(1) \longmapsto 4(1) \longmapsto 8(2) \longmapsto 9$ \\
\hline 12101212111210121210 & $1(1) \longmapsto 2(1) \longmapsto 4(1) \longmapsto 8(2) \longmapsto 10(2) \longmapsto 20(2)$ \\
\hline 1210121211121010 & $1(1) \longmapsto 2(1) \longmapsto 4(1) \longmapsto 8(2) \longmapsto 10(2) \longmapsto 16(1)$ \\
\hline 1210121211121011 & $1(1) \longmapsto 2(1) \longmapsto 4(1) \longmapsto 8(2) \longmapsto 10(2) \longmapsto 16(2)$ \\
\hline $12101212111210 *$ & $1(1) \longmapsto 2(1) \longmapsto 4(1) \longmapsto 8(2) \longmapsto 10(2) \longmapsto 15$ \\
\hline
\end{tabular}


The first and second tree have components with periods respectively 5,$6 ; 19$, and 15, 16; 9, like Lemma 6.19 states. See also how both trees have the trunk 20,18,14, 12, 11 in accordance with Lemma 6.6. However, by comparing this example to 6.11 (Figure $\mathrm{H}$ ) one sees that the components with periods 12 and 11 in the first tree actually belong to a shadow tree of the 6-periodic component and are thus nonexistent. (In the second tree, all components do exist; we will see this in $\S 8$.)

The whole base component $\mathcal{C}$ together with the components with periods less than ten (5, 6 above the first sector and 9 above the second) make up the tree $\mathcal{S}$ defined for Corollary $6.21 ; \mathcal{S}$ is combinatorially equivalent to the shadow tree $\widetilde{\mathcal{V}}_{\text {./2 }}$ of $C$ and, by 6.16 , to all other shadow trees as well.

\begin{tabular}{|c|c|}
\hline 12101212121210121210 & $1(1) \longmapsto \ldots \longmapsto 8(2) \longmapsto 12(2) \succ 14(1) \succ 18(2) \succ 20(1)$ \\
\hline 1210121212121010 & $1(1) \longmapsto \ldots \longmapsto 8(2) \longmapsto 12(2) \longmapsto 14(1) \longmapsto 16(1)$ \\
\hline 1210121212121011 & $1(1) \longmapsto \ldots \longmapsto 8(2) \longmapsto 12(2) \longmapsto 14(1) \longmapsto 16(2)$ \\
\hline $12101212121210 *$ & $1(1) \longmapsto \ldots \longmapsto 8(2) \succ 12(2) \longmapsto 14(1) \longmapsto 15$ \\
\hline 12101212121210121211 & $1(1) \longmapsto \ldots \longmapsto 8(2) \longmapsto 12(2) \longmapsto 14(1) \longmapsto 18(2) \longmapsto 20(2)$ \\
\hline $121012121212101212 *$ & $1(1) \longmapsto \ldots \longmapsto 8(2) \longmapsto 12(2) \longmapsto 14(1) \longmapsto 18(2) \longmapsto 19$ \\
\hline
\end{tabular}

In the next section we study the widths of wakes of existing hyperbolic components and sectors; this information will tell us how much room there is for components in each visible tree, and thus will help to find which components do not exist.

\section{WAKE WIDTH}

The difference of the two external arguments $\theta_{1}, \theta_{2}$ of a hyperbolic sector $\mathcal{K}$ is called its width. Since the angles have the same period $k$ as $\mathcal{K}$, the width must be of the form $|\mathcal{K}|=\theta_{2}-\theta_{1}=w /\left(d^{k}-1\right)$ for some integer $w$.

7.1. Narrow sectors and components. In 3.2, we defined a narrow sector as one whose wake contains no components with less period. This is equivalent to the standard definition: The sector $\mathcal{K}$ is narrow if $w=1$. In order to contain a component with minimal period $m$, the wake of $\mathcal{K}$ must have width $w /\left(d^{k}-1\right)>(d-1) /\left(d^{m}-1\right)$; then $w=1$ if and only if $m=k+1$. A hyperbolic component is said to be narrow if all its sectors are.

The following result is fundamental for studying which of the components given by VTA are existent in the Mandelbrot sets, because it tells us how much space there is above each hyperbolic sector for other components, but in the general form it has been "folklore". The quadratic case is proved in [Sch-1] using Douady's Tuning Algorithm. Instead of generalizing that method, our proof will be based on a kind of global counting argument. The special case of the main cardioid in $\mathcal{M}^{2}$ was treated in detail in [Dev] using Farey numbers, like we will. (I was unaware of the work [Dev] until 
Peter Haïssinsky mentioned it while reading the manuscript of this article.) Another discussion of the main cardioid case, using dyadic expansions of the external angles, is found in [Ate].

7.2. TheOREM. Let $\mathcal{K}$ be a hyperbolic sector of $\mathcal{M}^{d}$ with period $k$ and width $w /\left(d^{k}-1\right)$. Then each satellite component $\mathcal{C}$ at internal angle $p / q$ has width

$$
\Delta\left(\frac{p}{q}\right)=\frac{w\left(d^{k}-1\right)}{d^{q k}-1} .
$$

7.3. LemMA. Let $\mathcal{K}$ be a hyperbolic sector with period $k$ and wake-width $w /\left(d^{k}-1\right)$; let the wake contain a hyperbolic component $\mathcal{A}$ with minimal period $m$. Then:

(1) $w=d r+1$ for some $r \in \mathbb{N}$.

(2) The number of components with period (dividing) $k$ in the wake of one sector of $\mathcal{A}$ is $r(k, m)=\sum_{1 \leq i \leq(k-1) / m} d^{k-i m-1}$.

(3) If the wake of $\mathcal{K}$ contains no other components with periods less than $k$, then $w=d(d-1) r(k, m)+1$.

Proof. (1) Let $r$ be the number of other components with period (dividing) $k$ in the wake of $\mathcal{K}$. By Structure Theorem 2.8, each component has exactly $d$ rays with angle denominator $d^{k}-1$ landing at its boundary, and every such ray lands at the boundary of some such component. Thus the wake must contain exactly $d r$ rays with angle denominator $d^{k}-1$, so the width has numerator $d r+1$.

(2) The $m$-periodic component $\mathcal{A}$ must be narrow because $m$ is minimal. The sector-wake therefore has width $1 /\left(d^{m}-1\right)$ and contains $d r(k, m)$ rays of the form $t /\left(d^{k}-1\right)$. Now $r(k, m)$ must be the maximal number for which

$$
\frac{d r(k, m)-1}{d^{k}-1}<\frac{1}{d^{m}-1} \Leftrightarrow d r(k, m)<1+d^{k-m}+d^{k-2 m}+d^{k-3 m}+\ldots
$$

Thus $r(k, m)$ is the integer part of $\sum_{i=1}^{\infty} d^{k-i m-1}$; hence $i \leq(k-1) / m$.

(3) Since $\mathcal{A}$ has $d-1$ sectors, its wake contains $(d-1) r(k, m)$ components with periods dividing $k$. The difference between the two ultimate external angles of all these is $[d(d-1) r(k, m)-1] /\left[d^{k}-1\right]$, so $w=d(d-1) r(k, m)+1$.

7.4. Lemma. The characteristic interval of any satellite orbit portrait $\Theta$ with orbit period $k$, ray period $q k$ and rotation number $p / q$ has length

$$
\Delta\left(\frac{p}{q}\right)=w\left(\frac{p}{q}\right) \cdot \frac{d^{k}-1}{d^{q k}-1}
$$

with some integer $w(p / q)$.

Proof. Any interval of length $\Delta$ is mapped by $\sigma_{d}^{n}$ into an interval of length $d^{n} \Delta$, possibly covering the image more than once. By 2.3 , the complementary intervals $I_{1}, \ldots, I_{q}$ of $A_{1}$ are mapped by $\sigma_{d}^{k}$ so that $\left|I_{j+p}\right|=$ 
$d^{k}\left|I_{j}\right| \bmod 1$ for all $j \in \mathbb{Z}_{q}$ (thus $\sigma^{k}\left(I_{j}\right)$ covers $I_{j+p}$ once more than it does in other points on the circle). Since $\sum_{j=1}^{q}\left|I_{j}\right|=1$, the total length must be a natural number

$$
\Delta+d^{k} \Delta+\ldots+d^{(q-1) k} \Delta=\Delta \cdot \frac{d^{q k}-1}{d^{k}-1} .
$$

Now it remains to show that this number $w(p / q)$ depends on neither $p$ nor $q$, and that the constant equals $w$.

7.5. Lemma. If $w(p / q) \neq w\left(p^{\prime} / q^{\prime}\right)$, then $\left|w(p / q)-w\left(p^{\prime} / q^{\prime}\right)\right| \geq d$.

Proof. Lemmas 7.3 and 7.4 imply that $w(p / q)\left(d^{k}-1\right)=d r-1$ and $w\left(p^{\prime} / q^{\prime}\right)\left(d^{k}-1\right)=d r^{\prime}-1$ for some $r, r^{\prime} \in \mathbb{N}$. The difference $\left|r-r^{\prime}\right|=$ $\left|w(p / q)-w\left(p^{\prime} / q^{\prime}\right)\right|\left(d^{k}-1\right) / d$ must also be a natural number, so $\mid w(p / q)-$ $w\left(p^{\prime} / q^{\prime}\right) \mid$ is a multiple of $d$.

7.6. Farey addition. A Farey sequence of order $n$ is the increasing sequence $F_{n}$ of all (reduced) fractions $p / q$ such that $0 \leq p \leq q \leq n$; for example,

$$
F_{7}=\frac{0}{1}, \frac{1}{7}, \frac{1}{6}, \frac{1}{5}, \frac{1}{4}, \frac{2}{7}, \frac{1}{3}, \frac{2}{5}, \frac{3}{7}, \frac{1}{2}, \frac{4}{7}, \frac{3}{5}, \frac{2}{3}, \frac{5}{7}, \frac{3}{4}, \frac{4}{5}, \frac{5}{6}, \frac{6}{7}, \frac{1}{1}
$$

([H-W] or [Cha]). If $p / q$ and $p^{\prime} / q^{\prime}$ are successive fractions in some $F_{n}$, then $\left(p+p^{\prime}\right) /\left(q+q^{\prime}\right)$ is called their mediant. Fractions in $F_{n+1} \backslash F_{n}$ are mediants of neighboring fractions in $F_{n}$ [Cha, Theorem I.8], so all rational numbers on $[0,1]$ are obtained by Farey mediants. Moreover, of three successive fractions in the same Farey sequence, the middle one is the mediant of the other two [Cha, Theorem I.9]. It follows that the mediant of two Farey-adjacent fractions has the minimal denominator of all fractions between them.

7.7. LEmma. If $p / q$ and $p^{\prime} / q^{\prime}$ are successive fractions in $F_{s}$ such that $q$ is a multiple of $q^{\prime}$, then $q^{\prime}=1$.

Proof. Now $p / q$ is the mediant of $p^{\prime} / q^{\prime}$ and its Farey-neighbor $t_{1} / r_{1}$ of some order less than $s$, so $q=n q^{\prime}=q^{\prime}+r_{1}$. Iterating this argument, we obtain a sequence $\left(r_{i}\right)$,

$$
r_{i}=(n-i) q^{\prime}=q^{\prime}+r_{i+1},
$$

of denominators of numbers Farey-adjacent to $p^{\prime} / q^{\prime}$ of decreasing order. The sequence stops at $r_{n-1}=q^{\prime}$. But the only Farey-adjacent pair of numbers with equal denominators are $0 / 1$ and $1 / 1$, so $q^{\prime}=1$.

7.8. Lemma. Let the internal angles $p / q$ and $p^{\prime} / q^{\prime}$ of respectively two points $c$ and $c^{\prime}$ in $\partial \mathcal{K}$ be successive fractions in $F_{n}$. Denote by $D$ the domain between the subwakes of $\mathcal{K}$ with roots at $c$ and $c^{\prime}$, bounded by $\partial \mathcal{K}$ and the two external rays $\mathcal{R}_{\varphi_{+}\left(\begin{array}{c}p \\ q\end{array}\right)}$ and $\mathcal{R}_{\varphi_{-}\left(\begin{array}{c}p_{q^{\prime}}^{\prime} \\ \text { ( }\end{array}\right)}$ landing at $c$ and $c^{\prime}$, respectively. The 
width of $D$ is then

$$
\delta_{q, q^{\prime}}=\varphi_{-}\left(\frac{p^{\prime}}{q^{\prime}}\right)-\varphi_{+}\left(\frac{p}{q}\right)=\frac{w\left(\frac{p+p^{\prime}}{q+q^{\prime}}\right)\left(d^{k}-1\right)}{\left(d^{q k}-1\right)\left(d^{q^{\prime} k}-1\right)} .
$$

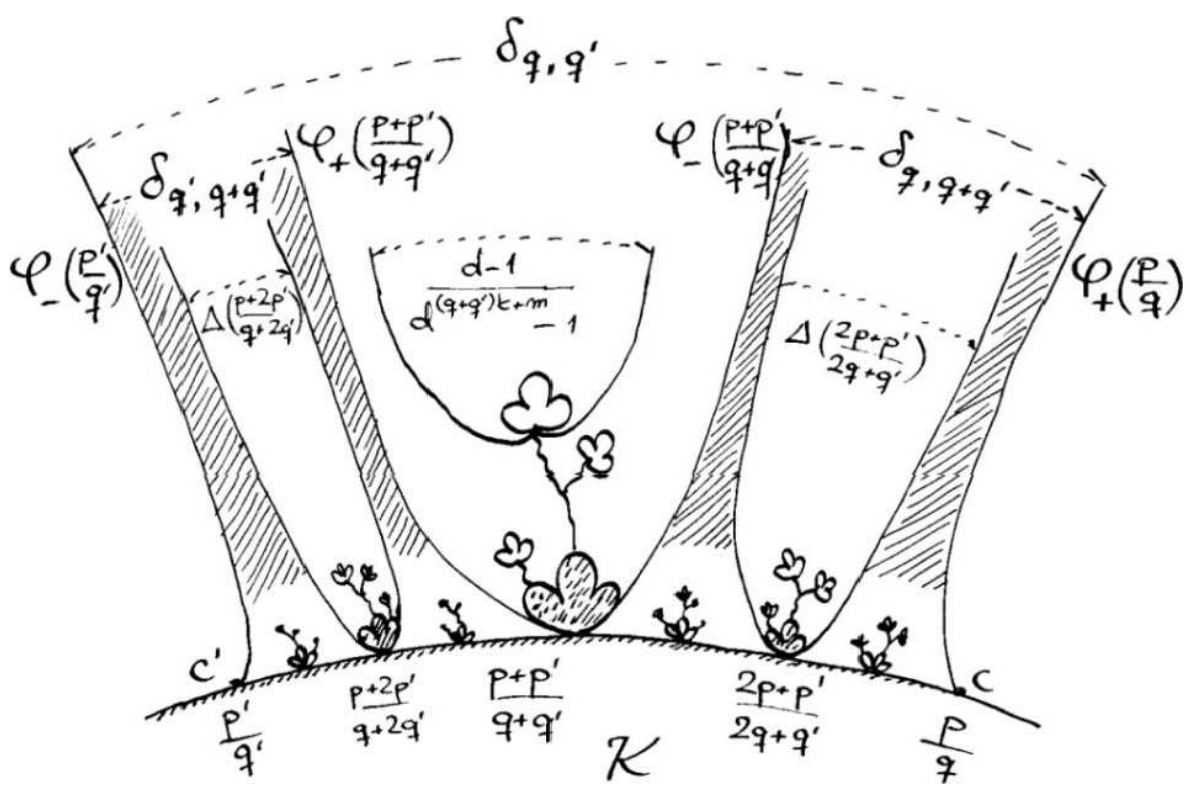

Fig. K

Proof. (See Figure K.) Because $\delta_{q, q^{\prime}}$ is the difference of two angles with periods $q k, q^{\prime} k$, the denominator must be as claimed. If $M$ is the smallest period of hyperbolic components in $D \backslash W\left(\left(p+p^{\prime}\right) /\left(q+q^{\prime}\right)\right)$, then the difference

$$
\begin{aligned}
\delta_{q, q^{\prime}}-\Delta\left(\frac{p+p^{\prime}}{q+q^{\prime}}\right) & =\frac{u}{\left(d^{q k}-1\right)\left(d^{q^{\prime} k}-1\right)}-\frac{v}{d^{\left(q+q^{\prime}\right) k}-1} \\
& =\frac{(u-v)+v\left(d^{-q k}+d^{-q^{\prime} k}\right)-\ldots}{d^{\left(q+q^{\prime}\right) k}-\ldots}
\end{aligned}
$$

must be positive and less than $2 /\left(d^{M-1}-1\right)$, because neither side of the middle wake can contain rays with periods $M-1$ or less. By Lemma 7.4,

$$
v=w\left(\frac{p+p^{\prime}}{q+q^{\prime}}\right)\left(d^{k}-1\right) .
$$

The $M$-periodic component sits in some visible tree $\mathcal{T}_{\cdot / r}$ of $\mathcal{K}$. By Lemmas 6.16 and 6.17 , the formal tree stemming at internal angle with denominator $r$ contains primitive addresses of lengths $m^{\prime}:=(r-2) k+m$ where $3 \leq m<2 k$. In particular, if $m$ is the minimal length of addresses visible from $\mathcal{K}$ given 
by VTA (6.4), then $M \geq m^{\prime}$. (We need not assume that the components with periods $m$ and $m^{\prime}$ actually exist.)

The minimal denominator $r$ of all internal angles for satellites in $D \backslash$ $W\left(\left(p+p^{\prime}\right) /\left(q+q^{\prime}\right)\right)$ belongs to the mediant of $\left(p+p^{\prime}\right) /\left(q+q^{\prime}\right)$ and either $p / q$ or $p^{\prime} / q^{\prime}$, so

$$
\begin{gathered}
r=\min \left\{2 q+q^{\prime}, q+2 q^{\prime}\right\} \geq q+q^{\prime}+2, \\
M \geq m^{\prime}=(r-2) k+m \geq\left(q+q^{\prime}\right) k+3 .
\end{gathered}
$$

We now have the upper bound

$$
\frac{2}{d^{M-1}-1}<\frac{2}{d^{\left(q+q^{\prime}\right) k+2}-1} \leq \frac{1}{d^{\left(q+q^{\prime}\right) k+1}-d^{-1}}<\frac{1}{d^{\left(q+q^{\prime}\right) k}}
$$

for the difference above. This is only possible if $u=v$.

7.9. Lemma. For all $q \in \mathbb{N}, w(1 / q)=w=w((q-1) / q)$.

Proof. Angles $1 / q$ and $(q-1) / q$ are Farey-adjacent to $0 / 1$ and $1 / 1$, respectively, so we may use the previous result 7.8 for $q^{\prime}=1$. We prove the lemma for $1 / q$; the other case is similar.

The $d$-tupling map iterated $k$ times fixes the two angles $\theta_{ \pm}$bounding the sector $\mathcal{K}$, and maps the less characteristic angle of the $1 / q$-satellite component to the greater one. Now $\varphi_{+}^{0 / 1}=\theta_{-}\left(\right.$and $\varphi_{-}^{1 / 1}=\theta_{+}$), so using 7.4 we have

$$
\varphi_{-}^{1 / q}-\delta_{1, q}=\theta_{-}=\sigma^{k}\left(\theta_{-}\right)=\sigma^{k}\left(\varphi_{-}^{1 / q}-\delta_{1, q}\right)=\varphi_{+}^{1 / q}-d^{k} \delta_{1, q},
$$

which implies

$$
\Delta\left(\frac{1}{q}\right)=\varphi_{+}^{1 / q}-\varphi_{-}^{1 / q}=\left(d^{k}-1\right) \delta_{1, q} \quad \text { and so } \quad \delta_{1, q}=\frac{w(1 / q)}{d^{q k}-1} .
$$

In particular, for $q=2, \delta_{1,2}=w(1 / 2) /\left(d^{2 k}-1\right)$. Now

$\theta_{+}-\theta_{-}=\frac{w}{d^{k}-1}=\Delta\left(\frac{1}{2}\right)+2 \delta_{1,2}=\frac{w(1 / 2)\left(d^{k}-1\right)+2 w(1 / 2)}{d^{2 k}-1}=\frac{w(1 / 2)}{d^{k}-1}$,

so $w(1 / 2)=w$. On the other hand, Lemma 7.8 implies

$$
\delta_{1, q}=\frac{w((1+0) /(q+1))}{d^{q k}-1},
$$

which, together with $(7.9 \mathrm{a})$, yields $w(1 /(q+1))=w(1 / q)$. The claim now follows by induction with respect to $q$.

7.10. Lemma. For all $q \in \mathbb{N}$ and $p=2, \ldots, q-2, w(p / q)=w$.

Proof. Every rational number is obtained by the Farey procedure. Assume that

$$
\delta_{q, q^{\prime}}=\frac{w\left(d^{k}-1\right)}{\left(d^{q k}-1\right)\left(d^{q^{\prime} k}-1\right)} \quad \text { and } \quad \Delta\left(\frac{p+p^{\prime}}{q+q^{\prime}}\right)=\frac{w\left(d^{k}-1\right)}{d^{\left(q+q^{\prime}\right) k}-1}
$$


for some pair of successive numbers $p / q$ and $p^{\prime} / q^{\prime}$ in $F_{n}$. By 7.9 , this is true at least when $n=1$.

For the induction step, we calculate the widths $\delta_{q, q+q^{\prime}}$ and $\delta_{q^{\prime}, q+q^{\prime}}$ of the domains that should contain the two mediant subwakes next to the one at internal angle $\left(p+p^{\prime}\right) /\left(q+q^{\prime}\right)$ (Figure $\mathrm{K}$ ). By Lemma 7.3, the numerators differ from $w$ by a multiple of $d$, if at all. Hence for some $i, j \in \mathbb{Z}$,

$$
\begin{aligned}
\delta_{q, q+q^{\prime}}+\delta_{q^{\prime}, q+q^{\prime}}= & \frac{(w+i d)\left(d^{k}-1\right)}{\left(d^{\left(q+q^{\prime}\right) k}-1\right)\left(d^{q k}-1\right)}+\frac{(w+j d)\left(d^{k}-1\right)}{\left(d^{\left(q+q^{\prime}\right) k}-1\right)\left(d^{q^{\prime} k}-1\right)} \\
= & \delta_{q, q^{\prime}}-\Delta\left(\frac{p+p^{\prime}}{q+q^{\prime}}\right) \\
& +\frac{d\left(d^{k}-1\right)}{d^{\left(q+q^{\prime}\right) k}-1} \cdot\left(\frac{i}{d^{q k}-1}+\frac{j}{d^{q^{\prime} k}-1}\right) .
\end{aligned}
$$

The last term must be zero. Unless $q$ is a multiple of $q^{\prime}$ (or vice versa), this implies $i=j=0$. Thus

$$
w\left(\frac{2 p+p^{\prime}}{2 q+q^{\prime}}\right)=w=w\left(\frac{p+2 p^{\prime}}{q+2 q^{\prime}}\right),
$$

as claimed.

If $q$ is a multiple of $q^{\prime}$, then by Lemma 7.7, $q^{\prime}=1$. All angles Fareyadjacent to $0 / 1$ or $1 / 1$ are of the form $1 / q$ or $(q-1) / q$, respectively. This case was already proved in 7.9 .

Proof of Theorem 7.2. Now $w(p / q)=w$ for all $p / q \in \mathbb{Q} \cap] 0,1[$.

The next result is another "weak" or "partial translation principle":

7.11. Corollary. Every visible tree $\mathcal{T}_{\cdot / q+1}$ of a sector $\mathcal{K}$ contains a subtree which is combinatorially equivalent to $\mathcal{T}_{\cdot / q}$.

Proof. Denote the wake of $\mathcal{K}$ by $W(\mathcal{K})$ and its width by $|W(\mathcal{K})|=$ $w /\left(d^{k}-1\right)$. Let $\mathcal{A}$ be the hyperbolic component with minimal period, $m$, in the wake $W^{q}$ of the $\cdot / q$-satellite component, and assume there is another component $\mathcal{B} \in W^{q} \backslash W(\mathcal{A})$ with period $n$. We will show that there are components $\mathcal{A}^{\prime} \in W^{q+1}$ and $\mathcal{B}^{\prime} \in W^{q+1} \backslash W\left(\mathcal{A}^{\prime}\right)$ with periods $\left\langle\mathcal{A}^{\prime}\right\rangle=k+m$ and $\left\langle\mathcal{B}^{\prime}\right\rangle=k+n$. The claim will then follow from Theorem 6.14 , because every visible tree is a subtree of the corresponding formal tree (by 6.4).

Theorem 7.2 now gives the width of the wake $W^{q}$; it must contain all the $d$ rays of angle period $m$ which land at $\partial \mathcal{A}$, so

$$
\frac{w\left(d^{k}-1\right)}{d^{q k}-1}>\frac{d-1}{d^{m}-1}
$$


and therefore

$$
\begin{aligned}
\frac{w\left(d^{k}-1\right)}{d^{(q+1) k}-1} & >\frac{(d-1)\left(d^{q k}-1\right)}{\left(d^{m}-1\right)\left(d^{q k+k}-1\right)}=\frac{d-1}{d^{k+m}-d^{k}+d^{k-q k+m}-\ldots} \\
& >\frac{d-1}{d^{k+m}-1} .
\end{aligned}
$$

Hence there are (at least) $d-1$ rays with angle period $(q-2) k+m=: m^{\prime}$ in $W^{q}$. None of them can have period $s$ strictly dividing $m^{\prime}$ : otherwise $W^{q}$ would have to contain a sector of width at least $1 /\left(d^{s}-1\right) \geq 1 /\left(d^{m^{\prime} / 2}-1\right)$. But because $m$ was minimal, $|W|=w /\left(d^{k}-1\right)<1 /\left(d^{m-1}-1\right)$, so

$$
\left|W^{q}\right|=\frac{w\left(d^{k}-1\right)}{d^{q k}-1}<\frac{\left(d^{k}-1\right)^{2}}{\left(d^{m-1}-1\right)\left(d^{q k}-1\right)}<\frac{1}{d^{m / 2} \cdot d^{(q-2) k / 2}}<\frac{1}{d^{m^{\prime} / 2}-1} .
$$

Each ray of angle period $m^{\prime}$ lands at the boundary of some hyperbolic component $\mathcal{A}^{\prime}$ with the same period, so the wake must contain such a component. Moreover, $\mathcal{A}^{\prime}$ has exactly $d$ rays landing at its boundary, and the wake must contain them all.

All the rays with angle period $n$ that land at $\partial \mathcal{B}$ must fit in $W^{q} \backslash W(\mathcal{A})$, SO

$$
\frac{w\left(d^{k}-1\right)}{d^{q k}-1}-\frac{d-1}{d^{m}-1}>\frac{u}{d^{n}-1}
$$

and hence

$$
\begin{aligned}
& \frac{w\left(d^{k}-1\right)}{d^{q k+k}-1}-\frac{d-1}{d^{k+m}-1} \\
& \quad>\frac{u\left(d^{q k}-1\right)}{\left(d^{n}-1\right)\left(d^{q k+k}-1\right)}+\frac{(d-1)\left(d^{q k}-1\right)}{\left(d^{m}-1\right)\left(d^{q k+k}-1\right)}-\frac{d-1}{d^{k+m}-1} \\
& \quad=\frac{u}{d^{k+n}-d^{k}+\ldots}+(d-1) \cdot\left(\frac{1}{d^{k+m}-d^{k}+\ldots}-\frac{1}{d^{k+m}-1}\right) \\
& >\frac{u}{d^{k+n}-1} .
\end{aligned}
$$

The respective domain $W^{q+1} \backslash W\left(\mathcal{A}^{\prime}\right)$ between wakes must thus contain at least the same number $u+1$ of rays with angle period $k+n$ as there were those with period $n$, so it must also contain the component $\mathcal{B}^{\prime}$ as claimed.

\section{TO BE OR NOT TO BE?}

In Sections 5 and 6 we found some reasons for a component not to exist: belonging to a shadow tree or sitting above another nonexistent component. Now we look at some reasons to exist. The special case of a narrow root component is well known. The quadratic case is proved in [L-S-1, 10.2]; we generalize it here for all $d$. 
8.1. THEOREM. If $\mathbf{c} \in \dot{\Sigma}_{k}^{d}$ is the kneading sequence of an existent narrow hyperbolic sector $\mathcal{K}$ and $1 \leq n \leq k$, then for every $q=2,3, \ldots$ all kneading sequences of the form $\overline{\left(c_{1} \ldots c_{k}\right)^{q-1} b_{1} b_{2} \ldots b_{n}}$ are realized by hyperbolic components above the q-satellites of $\mathcal{C}$.

Proof. The number of such kneading sequences is $d^{n-1}(d-1)$ (only $d-1$ choices of $b_{n}$ define a finite address; see 5.4). The number $N$ of parameter rays with angle denominator $d^{(q-1) k+n}-1$ in a $p / q$-subwake of $\mathcal{K}$ must be the multiple of $d$ which is nearest to the product of that denominator with the width of the subwake. By Theorem 7.2, this is

$$
\frac{\left(d^{k}-1\right)\left(d^{(q-1) k+n}-1\right)}{d^{q k}-1}=d^{n}-\varepsilon
$$

where $0<\varepsilon \leq 1$, so $N=d^{n}$. None of these rays has a proper divisor of $(q-1) k+n$ as its exact period, because the $p / q$-subwake cannot contain components with periods less than $(q-1) k+1$. Hence the number of hyperbolic sectors with period $(q-1) k+n$ is also $(d-1) d^{n-1}$.

To prove that none of these kneading sequences occurs twice, assume there are two components with period $(q-1) k+n$ in the same subwake sharing a kneading sequence. The combinatorial arcs joining them to $\mathcal{K}$ must diverge at a sector $\mathcal{B}$, at internal angles with denominators $r \geq 3$. The minimal period of components in a $\cdot / r$-subwake of $\mathcal{B}$ is at least

$$
(r-2)\langle\mathcal{B}\rangle+(q-1) k+1>2(q-1) k+2>(q-1) k+n,
$$

which is a contradiction. Hence every kneading sequence occurs exactly once.

Equivalently: for each $q$, all addresses of the form $C=1\left(s_{1}\right) \longmapsto \ldots \longmapsto$ $k\left(s_{k}\right)$ followed by any combination of increasing period numbers $(q-1) k+$ $1, \ldots, q k$ and sector numbers $1, \ldots, d-1$ are realized if $C$ is narrow.

8.3. Corollary. For all $q$, the visible $q$-tree of a narrow $k$-periodic hyperbolic sector $\mathcal{C}$ consists of $k$ visible components: one of each period $(q-1) k+1, \ldots, q k$. Hence, if $1\left(s_{1}\right) \longmapsto \ldots \longmapsto k\left(s_{k}\right)$ is the internal address of $\mathcal{C}$, then every address $1\left(s_{1}\right) \longmapsto \ldots \longmapsto k\left(s_{k}\right) \longmapsto n\left(s_{n}\right)$ with $n>k$ is realized.

We conclude that for narrow sectors our algorithm 6.4 gives the whole visible tree and nothing more.

Our definition of narrow formal address - one whose periodic word does not begin and end with the same subword of any length (6.3) - is justified by the following lemma:

8.4. Lemma. Narrow, existent hyperbolic sectors have narrow internal addresses. 
Proof. Let $\mathcal{B} \subset \mathcal{M}^{d}$ be a narrow sector with period $n$. If its internal address $B$ were not narrow, the algorithm would yield a formal address $A$ with length less than $n$ above $B$. Since $\mathcal{B}$ was narrow, $A$ cannot be realized. But by 8.3 , VTA only gives realizable addresses, so not even nonexistent components with periods less than $n$ can occur above $\mathcal{B}$. Thus $B$ must be a narrow address.

The next lemma gives another sufficient condition for existence, independent of the degree $d$. Figure L illustrates the proof.
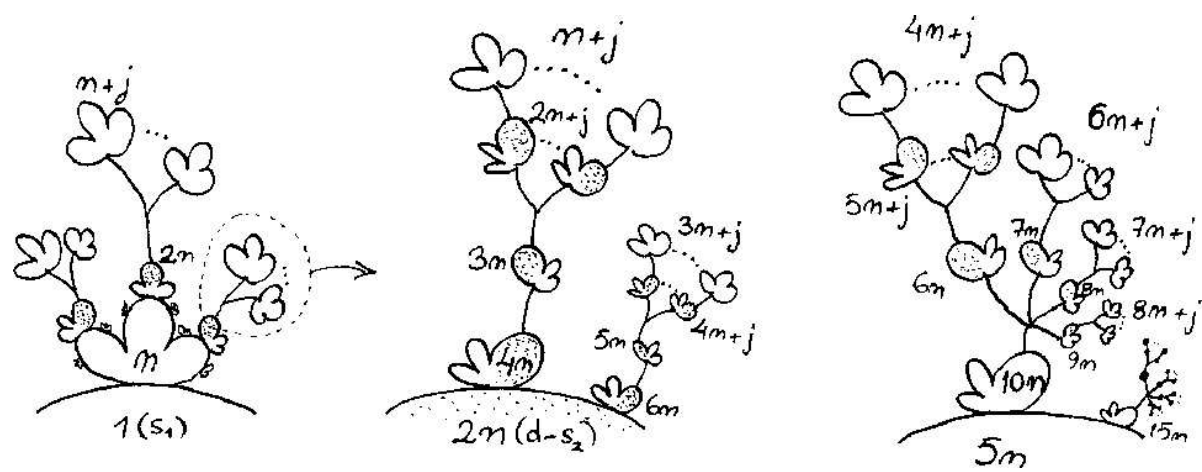

Fig. L

8.5. Lemma. For all d, every formal address with at most four steps is realized by a hyperbolic component in $\mathcal{M}^{d}$.

Proof. Consider an address of the form $1\left(s_{1}\right) \longmapsto n_{2}\left(s_{2}\right) \longmapsto n_{3}\left(s_{3}\right) \longmapsto n_{4}$; to simplify notation, we set $n:=n_{2}$. The address $1\left(s_{1}\right) \longmapsto n\left(s_{2}\right)$ corresponding to the kneading sequence $\overline{\mathbf{s}_{1}^{n-1}\left(\mathbf{s}_{1}+\mathbf{s}_{2}\right)}$ is realized by a satellite of the main epicycloid with any numbers $n \in \mathbb{N}$ and $\left\{s_{1}, s_{2}\right\} \subset\{1, \ldots, d-1\}$; for example, $K^{+}\left(1 / d^{n}-1\right)=\overline{\mathbf{1 1 \ldots 1 2}}$. These satellite components are obviously all narrow, so by 8.2 all components with addresses $1\left(s_{1}\right) \longmapsto n\left(s_{2}\right) \longmapsto n_{3}$ exist.

To find out which sectors at the next step are narrow, we construct the trees stemming at each sector of the satellite's satellites, $1\left(s_{1}\right) \longmapsto n\left(s_{2}\right) \longmapsto$ $q n\left(s_{3}\right)$. This address has periodic word $\mathbf{s}_{1}^{n-1}\left(\mathbf{s}_{1}+\mathbf{s}_{2}\right) \mathbf{s}_{1}^{n-1}\left(\mathbf{s}_{1}+\mathbf{s}_{2}+\mathbf{s}_{3}\right)$, so it is narrow unless $s_{3}=d-s_{2}$ (for $d=2$, there is no choice because $\left.s_{3}=s_{i}=1 \forall i\right)$. In this nonnarrow case, the word begins and ends with subwords consisting of $\mathbf{s}_{1}$ 's, of lengths $n-1, \ldots, 2,1$. Thus the formal $\cdot / q$ tree has a fan-like shape, consisting of $n-1$ primitive components with periods $(q-1) n+1, \ldots, q n-1$. All their sectors have periodic words starting with $\mathbf{s}_{1}^{n-1}\left(\mathbf{s}_{1}+\mathbf{s}_{2}\right)$, followed by a number of $\mathbf{s}_{1}$ 's, followed by another figure $\left(\mathbf{s}_{1}+\mathbf{s}_{3}\right) \neq \mathbf{s}_{1}$, so the primitive components are all narrow. 
Theorem 8.1 now implies that all four-step addresses are realizedexcept, possibly, if $n_{3}\left(s_{3}\right)=p n\left(d-s_{2}\right)$ for some $p$. We first consider the case $p=2$.

All components between the satellite $\mathcal{C} \sim 1\left(s_{1}\right) \longmapsto n\left(s_{2}\right) \longmapsto 2 n\left(d-s_{2}\right)$ and any of the narrow primitive components with period $n+j(1 \leq j<n)$ are found by comparing the kneading sequences as usual (LSA, 4.9). The sequences $\overline{\mathbf{s}_{1}^{n-1}\left(\mathbf{s}_{1}+\mathbf{s}_{2}\right) \mathbf{s}_{1}^{n}} \in \dot{\Sigma}_{2 n}^{d}$ and $\overline{\mathbf{s}_{1}^{n-1}\left(\mathbf{s}_{1}+\mathbf{s}_{2}\right) \mathbf{s}_{1}^{j}} \in \Sigma_{n+j, \infty}^{d}$ first differ at index $2 n+j$. For any $j$, the next two differences occur at indices $3 n$ and $4 n$. Hence there do exist $2 n$ components with periods $n_{4}=n+1, \ldots, 2 n-1$, $2 n+1, \ldots, 3 n, 4 n$ in $\mathcal{T}_{\cdot / 2}(\mathcal{C})$. Using Lemma 6.16 , we find all other trees; there are components with periods $n_{4}=3 n+1, \ldots, 4 n-1,4 n+1, \ldots, 5 n, 6 n$ in $\mathcal{T}_{\cdot / 3}(\mathcal{C})$, and so forth. Thus every address $1\left(s_{1}\right) \longmapsto n\left(s_{2}\right) \longmapsto 2 n\left(s_{3}\right) \longmapsto n_{4}$ is realized.

The case $p \geq 3$ is more complicated. Each formal tree (grown by VTA, 6.4) of the satellite $\mathcal{B}=1\left(s_{1}\right) \longmapsto n\left(s_{2}\right) \longmapsto p n\left(d-s_{2}\right)$ contains a subtree that is combinatorially equivalent to the trees $\mathcal{T}_{. / q}(\mathcal{C})$; the existence of its $n-1$ branch-tips with periods $(p-1) n+j$ is guaranteed by Corollary 7.11. But the formal $q$-tree also has $q-2$ other branches: tips with periods $(p+i) n+j$ and one with period $(p+1+i) n$ below them $(1 \leq i \leq q-2,1 \leq j<n)$. Because all components with period equal to that of the base $\mathcal{B}$ (i.e. to $p n$ ) sit above the treetops, their existence can be shown by checking that the sum of their wake-widths is less than $|W(\mathcal{B})|=(d-1)\left(d^{n}-1\right) /\left(d^{p n}-1\right)$. The existence of the other components in the formal tree then follows; there are $2(n-1)+1+(q-2) n+1=q n$ of them, as there should be by 6.17 . We conclude that the claimed address is indeed realized, even if $n_{3}$ is a multiple of $n=n_{2}$.

8.6. REMARK. This result cannot be improved, because there are nonexistent components with internal addresses of five steps; for example in $\Lambda^{2}$,

$$
\begin{array}{ll}
1 \longmapsto 2 \longmapsto 4 \longmapsto 5 \longmapsto 6 & \quad \text { (shadow satellite of } A \in \Lambda_{3}^{2} \text { ), } \\
1 \longmapsto 3 \longmapsto 6 \longmapsto 7 \longmapsto 8 & \text { (shadow satellite of } B \in \Lambda_{4}^{2} \text { ), } \\
1 \longmapsto 2 \longmapsto 4 \longmapsto 8 \longmapsto 9 & \text { (in a shadow tree of } C \in \Lambda_{6}^{2} \text { ). }
\end{array}
$$

Now we know that the whole tree in Example 6.11 (Figure G) exists: the base sector exists because its internal address $1(1) \longmapsto 3(1) \longmapsto 6(1) \longmapsto 7(1)$ has only four steps. Its periodic word is $\mathbf{1 1 2 1 1 0 2}$, so the sector is narrow; by 8.3 , every address in any of its trees is realized in $\mathcal{M}^{3}$. This same kneading sequence is also realized for any degree $d$.

The base component in Example 6.22 (left of Figure $\mathrm{J}$ ) at internal address $1(1) \longmapsto 2(1) \longmapsto 4(1) \longmapsto 8(2) \longmapsto 10$ exists, because its first sector is a subaddress of the infinite root address of the six-periodic component at internal address $A=1(1) \longmapsto 2(1) \longmapsto 4(1) \longmapsto 6$; $\widehat{\varkappa}_{3}(A)=\overline{\mathbf{1 2 1 0 1 2}}$, and this 
gets mapped to

$$
1(1) \longmapsto 2(1) \longmapsto 4(1) \longmapsto 8(2) \longmapsto 10(1) \longmapsto 14(2) \longmapsto 16(1) \longmapsto 20(2) \longmapsto \ldots
$$

As we saw in previous examples, a formal tree may branch in two ways: either within a component (so the various combinatorial arcs diverge from different sectors) or elsewhere. This latter type means that there are several subwords beginning and ending the periodic word of one sector. Nonexistent components can only occur above this second type of branching points: if a nonnarrow sector $\mathcal{B}$ exists, then there must exist some components with periods less than $\langle\mathcal{B}\rangle$ above it. All possible periods are given by VTA. Hence:

8.7. LEMMA. If there is only one number $l$ such that $l$ first and last digits in the periodic word of an existing sector $\mathcal{B}$ coincide, then the component immediately visible over $\mathcal{B}$ with period $\langle\mathcal{B}\rangle-l$ exists as well.

Lemma 8.7 now guarantees the existence of all components in the second 2 -tree in 6.22 , and also in the first 2-tree except the ones in the shadow tree of $A$ (with periods 12 and 11).

Now we are ready for one of the main results: even if some component is nonexistent, it "becomes existent" by increasing the degree $d$ and picking right sector numbers.

8.8. Theorem. Every formal rough address $A$ of length $n$ or less is realized in $\mathcal{M}^{d}$ by some combination of sector numbers if $d \geq n / 2$.

We need one more lemma to prove this. The idea is shown in Figure M, where $n_{k}-k=9-5=4$ and $d=4,5,6,7$.
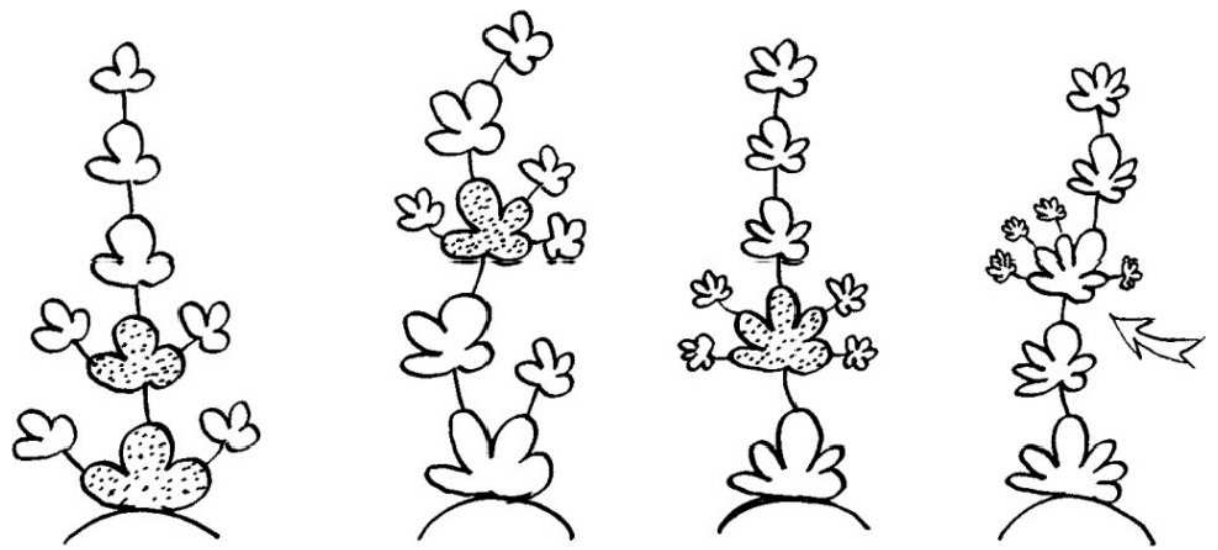

Fig. M

8.9. LemMA. If a hyperbolic component $\mathcal{N}$ with internal address $N=$ $1\left(s_{1}\right) \longmapsto \ldots \longmapsto n_{k}\left(s_{k}\right) \longmapsto n_{k+1}$ exists in $\mathcal{M}^{d}$ with $d \geq n_{k}-k+3$, then at least one of its sectors is narrow. 
Proof. Each visible tree $\mathcal{T}_{\cdot / q}$ of the sector $\mathcal{K}$ realizing the subaddress $K=1\left(s_{1}\right) \longmapsto \ldots \longmapsto n_{k}\left(s_{k}\right)$ contains (at most) $n_{k}$ hyperbolic components, by 6.17 . One of them is $\mathcal{N}$.

If none of $\mathcal{N}$ 's sectors is narrow, then every sector has a component with period less than $n_{k+1}$ behind it. These are all visible from $\mathcal{K}$, because $\mathcal{N}$ is. But then we would have at least $d$ components in the tree, namely $\mathcal{N}$ and one above each of its $d-1$ sectors, so $d \leq n_{k}$.

We deal with the case $n_{k}-k+3 \leq d \leq n_{k}$ using Lemma 6.6: the formal tree $\widetilde{\mathcal{T}}_{. / q}$ contains a chain of $k$ components (some of which may be nonexistent). Because this secondary trunk can intersect at most one of $\mathcal{N}$ 's sector wakes, now actually $k+d-2 \leq n_{k}$. This contradicts the assumption, so some sector must be narrow.

Proof of Theorem 8.8. Let $B:=1\left(s_{1}\right) \longmapsto n_{2}\left(s_{2}\right) \longmapsto \ldots \longmapsto n_{k}\left(s_{k}\right)$ be the last subaddress of $A=B \longmapsto n$. Corollary 8.3 and Lemma 8.9 together make an induction argument to find an existent component of period $n_{j}$ at each step $j=1, \ldots, k$.

There is some set of sector numbers $s_{j}$ such that $B$ is a narrow sector of such a component, as long as $d \geq n_{k-1}-(k-1)+3=n_{k-1}-k+4$. If $k<3$, then $\mathcal{B}$ is always narrow, so $\mathcal{A}$ exists for every $d$. Otherwise $k \geq 3$ and $n_{k} \geq n_{k-1}+1 \geq n_{k-1}+1-k+3$, so $d \geq n_{k}$ is a sufficient condition for $\mathcal{A}$ to exist.

By 8.2 , now also $B \longmapsto\left((p-1) n_{k}+1\right)\left(r_{1}\right) \longmapsto \ldots \longmapsto p n_{k}\left(r_{n_{k}}\right)$ is a realizable address for any integer $p>1$ and any set of $r_{i} \in\{0,1, \ldots, d-1\}$ (here $r_{i}=0$ means that the period $(p-1) n_{k}+i$ does not appear in the address, otherwise $r_{i}$ is the sector number of the respective subaddress).

When $p n_{k} \geq n$, the sufficient condition becomes $d \geq n / p$; this is satisfied if $d \geq n / 2$.

8.10. EXAMPLE. The shortest component address that is nonrealizable in the Mandelbrot set, $1(1) \longmapsto 2(1) \longmapsto 4(1) \longmapsto 5(1) \longmapsto 6 \in \Lambda_{6}^{2}$, belongs to the shadow two-satellite of the primitive three-periodic component and has kneading sequence $\overline{\mathbf{1 0 1 1 0 0}}$. In $\Lambda_{6}^{3}$, the same rough address comes in sixteen versions:

$$
\begin{aligned}
& \text { 12102* } 1(1) \longmapsto 2(1) \longmapsto 4(1) \longmapsto 5(1) \longmapsto 6 \quad \text { (purely narrow) } \\
& \text { 12100* } 1(1) \longmapsto 2(1) \longmapsto 4(1) \longmapsto 5(2) \longmapsto 6 \quad \text { (purely narrow) } \\
& \text { 12112* } * 1(1) \longmapsto 2(1) \longmapsto 4(2) \longmapsto 5(1) \longmapsto 6 \quad \text { (shadow satellite) } \\
& \text { 12110* } 1(1) \longmapsto 2(1) \longmapsto 4(2) \longmapsto 5(2) \longmapsto 6
\end{aligned}
$$

(and the same with $\left.\mathbf{s}_{1}\left(\mathbf{s}_{1}+\mathbf{s}_{2}\right)=\mathbf{1 0}, \mathbf{2 0}, \mathbf{2 1}\right)$. Only four of them have an infinite root address of a lesser-periodic primitive component $(\overline{\mathbf{1 2 1}}, \overline{\mathbf{1 0 1}}, \overline{\mathbf{2 0 2}}$, $\overline{\mathbf{2 1 2}})$ revealing them as shadow satellites. The remaining twelve components 
are existent, and eight of these are purely narrow (have all subaddresses narrow, as defined in [L-S-1]).

Note that (pure) narrowness is not a necessary condition for a continuation of an address to be realizable, and in many cases $d$ may thus be much less than $n / 2$. Above we discussed the two eleven-periodic components appearing in Example 6.22. Neither sector of the base component $1(1) \longmapsto 2(1) \longmapsto 4(1) \longmapsto 8(2) \longmapsto 10$ is narrow; the component at $1(1) \longmapsto 2(1) \longmapsto 4(1) \longmapsto 8(2) \longmapsto 10(2) \longmapsto 11$ exists while the one at $1(1) \longmapsto 2(1) \longmapsto 4(1) \longmapsto 8(2) \longmapsto 10(1) \longmapsto 11$ does not.

8.11. EXAmple. All the five rough addresses of length eight that are not realized in $\mathcal{M}_{2}$ also become existent when $d=3$. Two of them are above the six-periodic shadow satellite of the previous example, two are shadow satellites of four-periodic sectors, and one is a primitive component in a shadow tree of a five-periodic component. Here are a nonexistent and an existent version of each:

$$
\begin{aligned}
& 1011001 * 1(1) \longmapsto 2(2) \longmapsto 4(1) \longmapsto 5(2) \longmapsto 6(2) \longmapsto 8 \\
& 1210001 * 1(1) \longmapsto 2(1) \longmapsto 4(1) \longmapsto 5(2) \longmapsto 6(2) \longmapsto 8 \\
& 1011000 * \quad 1(1) \longmapsto 2(2) \longmapsto 4(2) \longmapsto 5(2) \longmapsto 6(2) \longmapsto 7(2) \longmapsto 8 \\
& 1210000 * \quad 1(1) \longmapsto 2(1) \longmapsto 4(1) \longmapsto 5(2) \longmapsto 6(2) \longmapsto 7(2) \longmapsto 8 \\
& 1101110 * \quad 1(1) \longmapsto 3(2) \longmapsto 6(1) \longmapsto 7(2) \longmapsto 8 \\
& 1101120 * 1(1) \longmapsto 3(2) \longmapsto 6(2) \longmapsto 7(2) \longmapsto 8 \\
& 1001100 * \quad 1(1) \longmapsto 2(2) \longmapsto 3(2) \longmapsto 5(1) \longmapsto 6(2) \longmapsto 7(2) \longmapsto 8 \\
& 1001200 * \quad 1(1) \longmapsto 2(2) \longmapsto 3(2) \longmapsto 5(2) \longmapsto 6(2) \longmapsto 7(2) \longmapsto 8 \\
& 1011110 * \quad 1(1) \longmapsto 2(2) \longmapsto 4(1) \longmapsto 6(1) \longmapsto 7(2) \longmapsto 8 \\
& 1012120 * \quad 1(1) \longmapsto 2(2) \longmapsto 4(2) \longmapsto 6(2) \longmapsto 7(2) \longmapsto 8
\end{aligned}
$$

The next result will narrow our search for shadow components; for each existing sector suspected to hide them behind it, it suffices to look at just one tree, namely the one with denominator 2. (Compare to [Kel, 3.78], which states that among the real trees in case $d=2$, the $1 / 2$-tree is the only one which may be combinatorially nonequivalent to other trees of the same base component.)

8.12. ThEOREM. If a shadow component $\mathcal{F}$ is visible from an existent sector $\mathcal{G}$, then $\mathcal{F}$ sits in the formal $\cdot / 2$-tree of $\mathcal{G}$.

Proof. Because $\mathcal{F}$ is visible from sector $\mathcal{G}$, their periods cannot be equal. If $\langle\mathcal{F}\rangle\langle\langle\mathcal{G}\rangle$, the claim follows directly from 6.15 . Thus, we assume that $\langle\mathcal{F}\rangle>\langle\mathcal{G}\rangle$. 
The nonexistent component $\mathcal{F}$ belongs to a shadow tree of some component $\mathcal{B}$; this $\mathcal{B}$ is also visible from $\mathcal{G}$ because $\langle\mathcal{B}\rangle\langle\langle\mathcal{F}\rangle$, and if there were another component between $\mathcal{G}$ and $\mathcal{B}$ with period less than that, $\mathcal{F}$ would also be above it and thus invisible from $\mathcal{G}$.

Hence (by 6.4) the root sequences $\hat{\varkappa}(F)$ and $\hat{\varkappa}(B)$ agree with $\varkappa(G)$ for at least the first $\langle\mathcal{F}\rangle(>\langle\mathcal{G}\rangle)$ figures. The periodic root word of $\mathcal{F}$ is

$$
g_{1} \ldots g_{\langle\mathcal{B}\rangle} \ldots g_{\langle\mathcal{F}\rangle}= \begin{cases}\left(b_{1} \ldots b_{\langle\mathcal{B}\rangle}^{\prime}\right)^{p} & \text { if } p\langle\mathcal{B}\rangle=\langle\mathcal{F}\rangle, \\ \left(b_{1} \ldots b_{\langle\mathcal{B}\rangle}^{\prime}\right)^{p-1} b_{1} \ldots b_{r} & \text { if }\langle\mathcal{B}\rangle \nmid\langle\mathcal{F}\rangle,\end{cases}
$$

for some $p \geq 2, r=1, \ldots\langle\mathcal{B}\rangle-1$.

In case $\langle\mathcal{B}\rangle \nmid\langle\mathcal{F}\rangle$ there is a component $\mathcal{A} \succ \mathcal{B}$ such that $\langle\mathcal{A}\rangle=r$, by 6.21 . Now $\mathcal{A}, \mathcal{B}, \mathcal{F}$ all belong to the same formal tree $\widetilde{\mathcal{T}}_{\cdot / q}(\mathcal{G})$, so their periods must be between $(q-2)\langle\mathcal{G}\rangle$ and $q\langle\mathcal{G}\rangle$, by 6.17. Therefore

$$
q\langle\mathcal{G}\rangle>\langle\mathcal{F}\rangle=(p-1)\langle\mathcal{B}\rangle+\langle\mathcal{A}\rangle>2(q-2)\langle\mathcal{G}\rangle \text {, hence } q<4 .
$$

In case $\mathcal{F}$ is a shadow satellite of $\mathcal{B},\langle\mathcal{F}\rangle \geq 2\langle\mathcal{B}\rangle$, which leads to the same conclusion. It remains to rule out the possibility that $q=3$.

If $\mathcal{A}, \mathcal{B}, \mathcal{F} \in \widetilde{\mathcal{T}}_{\cdot / 3}(\mathcal{G})$, then Lemma 6.14 implies that the tree $\widetilde{\mathcal{T}}_{\cdot / 2}(\mathcal{G})$ contains components $\mathcal{A}^{\prime}, \mathcal{B}^{\prime}, \mathcal{F}^{\prime}$ so that $\mathcal{A}^{\prime} \succ \mathcal{B}^{\prime}$, and with periods $\langle\mathcal{A}\rangle-\langle\mathcal{G}\rangle$, $\langle\mathcal{B}\rangle-\langle\mathcal{G}\rangle,\langle\mathcal{F}\rangle-\langle\mathcal{G}\rangle$, respectively. By 6.19 , the shadow tree $\widetilde{\mathcal{V}} \cdot / p\left(\mathcal{B}^{\prime}\right)$ contains a component $\mathcal{F}^{\prime \prime}$ with period

$$
\begin{aligned}
\left\langle\mathcal{F}^{\prime \prime}\right\rangle & =(p-1)\left\langle\mathcal{B}^{\prime}\right\rangle+\left\langle\mathcal{A}^{\prime}\right\rangle=(p-1)\langle\mathcal{B}\rangle+\langle\mathcal{A}\rangle-p\langle\mathcal{G}\rangle \\
& =\left\langle\mathcal{F}^{\prime}\right\rangle-(p-1)\langle\mathcal{G}\rangle .
\end{aligned}
$$

(The shadow satellite case is similar, just substitute $\langle\mathcal{B}\rangle$ for $\langle\mathcal{A}\rangle$ in the calculation.) Because this number must be positive and $\left\langle\mathcal{F}^{\prime}\right\rangle\langle 2\langle\mathcal{G}\rangle$, it follows that $p=2$ and $\left\langle\mathcal{F}^{\prime \prime}\right\rangle\left\langle\langle\mathcal{G}\rangle\right.$. Thus (by 6.12) $\mathcal{F}^{\prime \prime}$ is visible from $\mathcal{G}$. But then the tree $\widetilde{\mathcal{T}}_{\cdot / 2}(\mathcal{G})$ contains two components whose periods differ by exactly $\langle\mathcal{G}\rangle$, which contradicts 6.17 . Hence necessarily $q=2$.

Finally, we show a couple of results concerning the properties of hyperbolic sectors with same kneading sequences but in Mandelbrot sets of different degrees.

8.13. Corollary. If a kneading sequence $\mathbf{b} \in \dot{\Sigma}_{n}^{d}$ refers to a shadow sector $\mathcal{B}$ of $\mathcal{M}^{d}$, and $D>d$, then $\mathbf{b}$ is not realized in $\mathcal{M}^{D}$ either.

Proof. (The proposition makes sense because $\dot{\Sigma}_{n}^{d} \subset \dot{\Sigma}_{n}^{D}$, by 5.6.)

Assume first that $\mathcal{B}$ is a sector of a shadow satellite, so the sequence $\mathbf{b}$ is $\overline{\left(c_{1} \ldots c_{k}\right)^{q-1} c_{1} \ldots\left(c_{k}+s\right)}$ for some divisor $k$ of $n$ and $s \in\{1, \ldots, d-1\}$ such that $\overline{c_{1} \ldots c_{k}} \subset \Sigma_{k, \infty}^{d}$. Then $c_{n_{i}+1} \ldots c_{k}=c_{1} \ldots c_{k-n_{i}}$ for the length $n_{i}$ of the last subaddress of $B$. This implies that $\overline{c_{1} \ldots c_{k}} \subset \Sigma_{k, \infty}^{D}$ for any degree $D>d$, so $\varkappa_{D}^{-1}(\mathbf{b})=: B^{\prime}$ refers to a shadow satellite sector $\mathcal{B}^{\prime}$ of $\mathcal{M}^{D}$. 
Otherwise $\mathcal{B}$ sits above some shadow satellite sector of $\mathcal{M}^{d}$, to which the same argument applies; by Theorem $6.14, \mathcal{B}^{\prime}$ is a shadow sector of any Mandelbrot set of higher degree as well.

8.14. Corollary. Let a kneading sequence $\overline{c_{1} \ldots c_{k}} \in \dot{\Sigma}_{k}^{d}$ be realized in both $\mathcal{M}^{d}$ and $\mathcal{M}^{d+1}$. Then for all $q \geq 2$ and $n \in\{1, \ldots, k\}$, the component $\mathcal{B}$ with kneading sequence $\overline{\left(c_{1} \ldots c_{k-1} \mathbf{d}\right)^{q-1} c_{1} \ldots c_{n-1} *}$ is realized in $\mathcal{M}_{d+1}$.

Proof. The kneading sequence $\overline{c_{1} \ldots c_{k-1} \mathbf{d}}$ belongs to another sector of the same component $\mathcal{C} \subset \mathcal{M}^{d+1}$, which was assumed to exist. This sector is obviously narrow, because the digit $\mathbf{d}$ does not appear in $\Sigma_{k}^{d}$ and hence no subword can be found at both ends of the periodic word. Now the existence of $\mathcal{B}$ follows from Corollary 8.3.

8.15. Remarks. It remains open whether all nonexistent components are shadow components for degrees $d>2$ (if not, the vanishing point of some nonexistent component would not be at the root of some primitive hyperbolic component, but somewhere else in $\mathcal{M}^{d}$ ). Examples 8.10 and 8.11 show at least that the nonadmissibility conditions in [B-S] (Remark 5.22 here) are not sufficient for a component to nonexist in a higher-degree Mandelbrot set; the relevant condition must depend on the sector numbers.

If nonexistence of a component did imply its being a shadow component, then Theorem 8.12 would imply some stronger results:

- Nonexistent components can only be found in the 1/2-tree of the last subaddress.

- If $B=1\left(s_{1}\right) \longmapsto \ldots \longmapsto n_{k}\left(s_{k}\right)$ is realized and $n_{k+1} \geq 3 n_{k}$, then a hyperbolic component with internal address $B \longmapsto n_{k+1}$ exists as well.

In 8.14 it would then suffice to assume the sequence to be realized in $\mathcal{M}^{d}$; existence of a corresponding sector in $\mathcal{M}^{d+1}$ would follow from 8.13 because the argument would work in both directions. Hence, these two results would combine to the following:

- A kneading sequence consisting of $d$ different digits is realized either in all Mandelbrot sets of degree at least $d$, or in none of them.

- All formal component addresses not realizable in $\mathcal{M}^{d}$ become realized in $\mathcal{M}^{d+1}$ by some sector choice of the last subaddress.

The latter result would improve Theorem 8.8, implying that all rough addresses are realized by some set of sector numbers in $\mathcal{M}^{3}$.

\section{References}

[Ate] P. Atela, Bifurcations of dynamic rays in complex polynomials of degree two, Ergodic Theory Dynam. Systems 12 (1991), 401-423. 
[B-K] C. Bandt and K. Keller, Symbolic dynamics for angle-doubling on the circle II: Symbolic description of the abstract Mandelbrot set, Nonlinearity 6 (1993), 377-392.

[Bea] A. F. Beardon, Iteration of Rational Functions, Grad. Texts in Math. 132, Springer, 1991.

[B-S] H. Bruin and D. Schleicher, Symbolic dynamics of quadratic polynomials, preprint \#7 Institut Mittag-Leffler, 2002.

[C-G] L. Carleson and T. W. Gamelin, Complex Dynamics, Universitext, Springer, 1993.

[Cha] K. Chandrasekharan, Introduction to Analytic Number Theory, Grundlehren Math. Wiss. 148, Springer, 1968.

[Dev] R. L. Devaney, The Mandelbrot set and the Farey tree, Amer. Math. Monthly 106 (1999), 289-302.

[Do-1] A. Douady, Algorithms for computing angles in the Mandelbrot set, in: Chaotic Dynamics and Fractals, Notes Rep. Math. Sci. Engrg. 2, Academic Press, 1986, $155-168$.

[Do-2] -, Descriptions of compact sets in $\mathbb{C}$, in: Topological Methods in Modern Mathematics, Publish or Perish, 1993, 429-465.

[D-H] A. Douady et J. Hubbard, Étude dynamique des polynômes complexes. Parties I et II, Publ. Math. d'Orsay 84-2 et 85-4, Univ. Paris-Sud, 1984 et 1985.

[Ebe] D. Eberlein, Rational parameter rays of Multibrot sets, master's thesis, Technische Univ. München, 1999.

[H-W] G. H. Hardy and E. M. Wright, An Introduction to the Theory of Numbers, Oxford Univ. Press, 5th ed., 1979.

[Kah] L. Kahanpää, Kardioidi, Lecture Notes \# 34, Dept. of Math., Univ. of Jyväskylä, 1995 (in Finnish).

[Kau] V. Kauko, Trees of visible components in the Mandelbrot set, Fund. Math. 164 (2000), 41-60.

[Kel] K. Keller, Invariant Factors, Julia Equivalences and the (Abstract) Mandelbrot Set, Lecture Notes in Math. 1732, Springer, 2000.

[Lav] P. Lavaurs, Une description combinatoire de l'involution définie par $\mathcal{M}$ sur les rationnels à dénominateur impair, C. R. Acad. Sci. Paris Sér. I Math. 303 (1986), 143-146.

[L-S-1] E. Lau and D. Schleicher, Internal addresses in the Mandelbrot set and irreducibility of polynomials, preprint \#19, Inst. for Math. Sci., Stony Brook, 1994.

[L-S-2] —, - Symmetries of fractals revisited, Math. Intelligencer 18 (1996), no. 1, $45-51$.

[Mil-1] J. Milnor, Dynamics in One Complex Variable, Vieweg, 1999.

[Mil-2] - Periodic orbits, external rays, and the Mandelbrot set: an expository account, Astérisque 261 (2000), 277-333.

[Pen] C. Penrose, Quotients of the shift associated with dendrite Julia sets of quadratic polynomials, Ph.D. thesis, Warwick, 1990.

[Rie] J. Riedl, Arcs in Multibrot sets, locally connected Julia sets, and their construction by quasiconformal surgery, Ph.D. thesis, Technische Univ. München, 2001.

[Sch-1] D. Schleicher, Internal addresses in the Mandelbrot set and irreducibility of polynomials, Ph.D. thesis, Cornell Univ., 1994.

[Sch-2] -, Rational parameter rays of the Mandelbrot set, preprint \#13, Inst. for Math. Sci., Stony Brook, 1997.

[S-S] X. Sheng and M. J. Spurr, Symmetries of fractals, Math. Intelligencer 18 (1996), no. $1,35-42$. 
[Thu] W. Thurston, On the geometry and dynamics of iterated rational maps, preprint, Princeton Univ., 1985.

Department of Mathematics

P.O. Box 35

FIN-40014 University of Jyväskylä, Finland

E-mail: virpik@maths.jyu.fi

Received 15 June 2003;

in revised form 5 November 2003 